\title{
A taxonomic revision of the African genus Desplatsia Bocq. (Malvaceae - Grewioideae)
}

\author{
Julia WELLSOW ${ }^{1}$, Michelle HART ${ }^{2}$, Peter WILKIE ${ }^{3}$ \& David J. HARRIS ${ }^{4, *}$ \\ 1,2,3,4 Royal Botanic Garden Edinburgh, 20A Inverleith Row, Edinburgh EH3 5LR, Scotland, UK. \\ ${ }^{1}$ Systematic Botany and Mycology, Faculty of Biology, University of Munich (LMU) \\ Menzinger Str. 67, 80638 Munich, Germany. \\ *Corresponding author: dharris@rbge.org.uk \\ ${ }^{1}$ Email:wellsow@bio.lmu.de \\ 2Email:mhart@rbge.org.uk \\ ${ }^{3}$ Email: pwilkie@rbge.org.uk
}

\begin{abstract}
A taxonomic revision of Desplatsia Bocq. (Malvaceae s. lat. Juss., subfamily Grewioideae Hochr., tribe Grewieae Endl.) based on about 800 herbarium specimens is presented. Desplatsia is a genus of trees and shrubs found in tropical West and Central Africa and is characterized by subulately divided stipules, the absence of an androgynophore, stamens that are fused to a tube at the base, and large and distinctive fruits that are dispersed by elephants. Four species are recognized (D. subericarpa Bocq., D. chrysochlamys (Mildbr. \& Burret) Mildbr. \& Burret, D. dewevrei (De Wild. \& T.Durand) Burret and D. mildbraedii Burret) and 12 species names are placed into synonymy, two of which have been put into synonymy for the first time: $D$. floribunda Burret syn. nov. and $D$. trillesiana (Pierre ex De Wild.) Pierre ex A.Chev. syn. nov. All four species are widely distributed and their conservation status is assessed as Least Concern (LC). A key to the species, full species descriptions, illustrations, a specimen citation list and distribution maps are provided.
\end{abstract}

Keywords. Elephant dispersal, Cross River Terminus, Dahomey Gap, West Africa, Central Africa.

Wellsow J., Hart M., Wilkie P. \& Harris D.J. 2019. A taxonomic revision of the African genus Desplatsia Bocq. (Malvaceae - Grewioideae). European Journal of Taxonomy 584: 1-38. https://doi.org/10.5852/ejt.2019.584

\section{Introduction}

Desplatsia Bocq. (Malvaceae s. lat. Juss., subfamily Grewioideae Hochr., tribe Grewieae Endl.) is a genus of trees and shrubs found in tropical West and Central Africa forest. The genus was first validly published by Bocquillon (1866) and comprises four species.

Desplatsia was last revised by Burret (1926) within his comprehensive treatment of the Tiliaceae Juss. (now Grewioideae). In this treatment, he recognized six species recorded from tropical West and Central Africa: D. chrysochlamys (Mildbr. \& Burret) Mildbr. \& Burret, D. floribunda Burret syn. nov., D. subericarpa Bocq., D. trillesiana (Pierre ex De Wild.) Pierre ex A.Chev. syn. nov., D. dewevrei (De Wild. \& T.Durand) Burret and D. mildbraedii Burret. The genus has subsequently been treated in 
several African floras and checklists (Hutchinson \& Dalziel 1954; Irvine 1961; Wilczek 1963; Keay 1989; Hawthorne 1990; Lebrun \& Stork 1997; Cable \& Cheek 1998; Verdcourt 2001; Harris 2002; Cheek et al. 2004; Sosef et al. 2006; Harris \& Wortley 2008; Cheek et al. 2011). However, these treatments lack a revisionary approach, and some have raised doubts on the delimitation of certain Desplatsia species, such as D. trillesiana syn. nov. (Sosef et al. 2006), D. floribunda syn. nov. (Harris 2002) and D. mildbraedii (Verdcourt 2001). The Plant List (2013) lists five accepted names: D. subericarpa, $D$. chrysochlamys, $D$. dewevrei, D. mildbraedii and D. floribunda syn. nov. These discrepancies clearly showed the need for a revision of the genus Desplatsia.

Although Desplatsia has been considered closely related to the African genus Duboscia Bocq. (Burret 1926; Bayer \& Kubitzki 2003), recent molecular studies have shown that Desplatsia may be sister to Grewia L. (Brunken \& Muellner 2012) rather than to Duboscia (Wellsow et al. in prep.).

No recent revision of Grewioideae as a whole exists. Some smaller genera have been recently revised such as Duboscia (Hyam et al. 2012) and, for Peninsular Malaysia, Microcos L. (Chung \& Soepadmo 2011). However, the larger genera such as Grewia and Trichospermum Blume are still awaiting revision. In this context, the revision of Desplatsia is a step forward towards completing genus and species delimitations within the Grewioideae.

\section{Materials and methods}

Herbarium specimens of Desplatsia held at BM, BR, E, K, M and P were studied on visits to those herbaria. In addition, digital images of specimens were examined from HBG, L, U and WAG, altogether representing about 800 specimens. Herbarium acronyms follow the Index Herbariorum (Thiers, continuously updated http://sweetgum.nybg.org/science/ih/). All material cited was seen either as a specimen in a herbarium or as an image. Where possible, the specimens are cited using a stable HTTP URI (Hypertext Transfer Protocol Uniform Resource Identifier) hyperlink to the specimen codes in square brackets after the Index Herbariorum herbarium codes (following Hyam et al. 2012).

Morphological characters are described and measurements taken from dried herbarium material and from field information given on herbarium labels (when possible). This is supplemented by information from the species descriptions in the Flora of Tropical East Africa (Verdcourt 2001). Flowering and fruiting periods given are based on information from herbarium specimen labels.

The terminology in the descriptions follows Harris \& Woolf Harris (1994).

The key and descriptions were prepared in a way to include as many vegetative and field characters as possible to make the key useful not only to herbarium taxonomists, but also to field botanists.

Plant material studied is listed per country, then alphabetically per collector under each species. Localities are cited as given on the herbarium specimen labels. If no geographical coordinates were available, these localities were determined using the online Global Gazetteer ver. 2.3 (http://www.fallingrain.com/world/). Data cleaning was performed by checking location data for potential errors by plotting the collection sites on country maps. Data points in the ocean, in countries that did not match the country given on the herbarium sheet or from locations that the collector is known not to have collected from during the time period given were corrected following Hijmans et al. (1999). Whenever an accession seemed doubtful, the coordinates were checked against the location given on the specimen sheet and discrepancies resolved.

Phenetic morphological criteria to delimit species have been chosen for this study. Species are separated by a discontinuity in characters (Crisp \& Weston 1993). 
Proposed IUCN conservation assessments were assigned following the IUCN categories and criteria ver. 3.1 (IUCN 2012) and distribution data based on georeferenced specimens as given in the present publication. Extent of occurrence (EOO) and area of occupancy (AOO) were calculated using GeoCAT (Bachman et al. 2011). Full, formal assessments will be submitted to IUCN.

\section{Institutional abbreviations}

RBGE = Royal Botanic Garden Edinburgh, Edinburgh, UK

\section{Results}

\section{Characters}

Characters of particular importance for species delimitation in Desplatsia include: habit (height and diameter at breast height (DBH)) of mature trees, shape of stipules, leaf texture and color when dried, size of leaf teeth, nature of indumentum on twigs, leaves, petioles and fruits.

\section{Habit}

All species of Desplatsia are woody. They range from small trees or shrubs (D. subericarpa, 2-3($8) \mathrm{m}$ tall) to large trees (D. mildbraedii, up to $25 \mathrm{~m}$ tall). DBH of mature trees ranges from $2-10 \mathrm{~cm}$ (D. subericarpa) to $80 \mathrm{~cm}$ (D. mildbraedii). In the key to species we use the size of flowering and fruiting individuals. Other characters have to be used for immature individuals of all species.

\section{Twigs}

Desplatsia chrysochlamys is differentiated from other species by its dense, long, coarse, orange hairs on the twigs. At the other extreme, individuals of $D$. dewevrei can have glabrous twigs.

\section{Stipules}

Desplatsia is characterized by subulately divided, persistent stipules. Between species, the stipules differ from being finely divided into linear or filiform lobes (D. subericarpa) to being divided for only part of their length into ovate-lanceolate lobes (D. dewevrei). The stipules look superficially like those of some species of Grewia and Microcos, and occasionally species of those two genera are mis-identified as Desplatsia.

\section{Petioles}

All species are petiolate. While a pulvinus at each end of the petiole is a general character for Malvaceae s. lat., it is most pronounced for $D$. subericarpa, in which it is distinctly inflated in the upper part (see Figs 1-2). Petiole length is not useful in species delimitation. Petiole indumentum is of some use in species delimitation with $D$. mildbraedii being distinctive by the presence of both short and scattered long hairs on the petiole (see Fig. 1).

\section{Leaves}

Leaves in all species are simple and alternate. They sometimes have domatia on the lower side of the leaves formed by hairs in nerve axils. These are most often observed in $D$. dewevrei, sometimes in D. subericarpa, but never in D. mildbraedii and D. chrysochlamys.

Leaf tips range from distinctly acuminate (D. subericarpa) to acute or slightly acuminate. Leaf margins range from obscurely (D. subericarpa) to clearly toothed. Both characters are useful for species delimitation. For example, $D$. dewevrei is clearly distinguishable from all other species by its large (up to $1 \mathrm{~cm}$ ), jagged teeth (see Figs 2-3). 


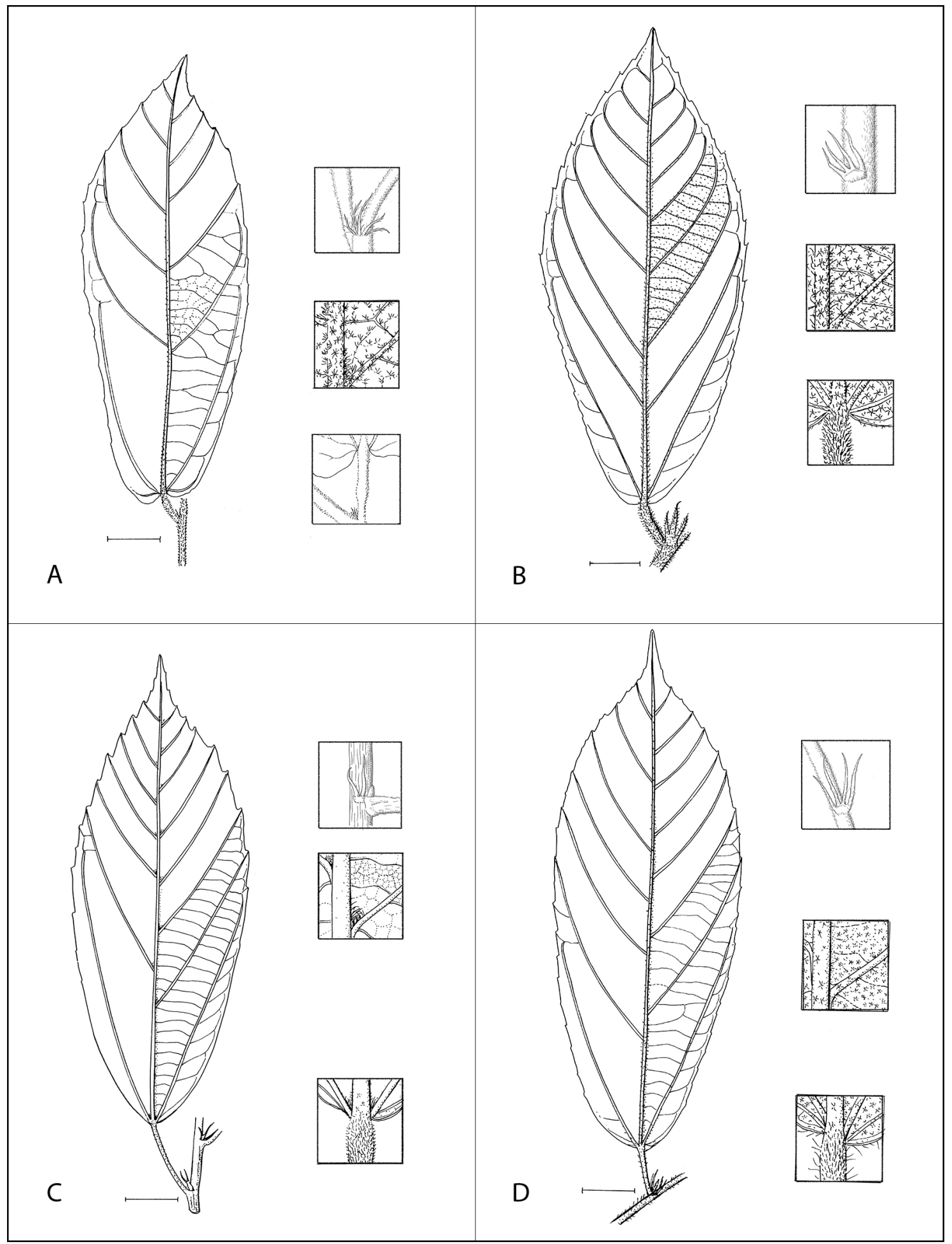

Fig. 1. Illustration of Desplatsia Bocq. leaves. A. D. subericarpa Bocq. (Harris 1387 (E)). B. D. chrysochlamys (Mildbr. \& Burret) Mildbr. \& Burret (Harris 5402 (E)). C. D. dewevrei (De Wild. \& T.Durand) Burret (Harris 7025 (E)). D. D. mildbraedii Burret (Harris 4397 (E)). Scale bars $=2 \mathrm{~cm}$. Boxed enlargements of petiole (in B-D) and midrib (A-D) are magnified $10 \times$ to represent the view through a typical $10 \times$ hand lens. Boxed enlargements of petiole (A) and stipules (A-D) are magnified $6 \times$. Images are taken from Harris \& Wortley (2008), illustrated by Rosemary Wise, with additional enlargements by Sanna Olander. 


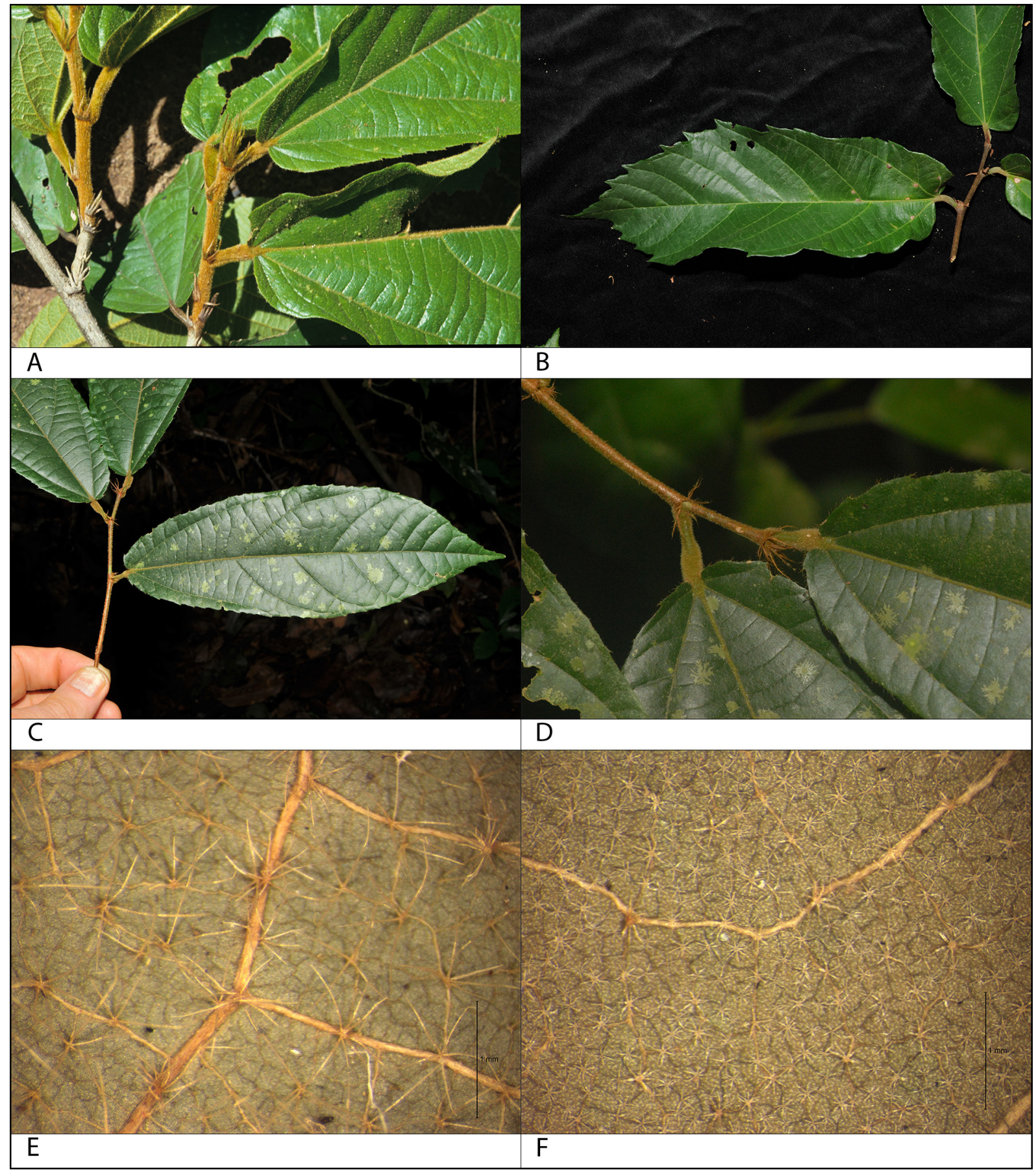

Fig. 2. Desplatsia Bocq. leaf images. A. D. chrysochlamys (Mildbr. \& Burret) Mildbr. \& Burret (Harris 4977 (E)). B. D. dewevrei (De Wild. \& T.Durand) Burret (Harris 9664 (E)). C-D. D. subericarpa Bocq. (Harris 10194). C. Leaves. D. Leaf base with subulately divided stipules and distinctly inflated petiole in upper part. E-F. Stellate hairs on leaf undersurface. E. D. chrysochlamys (Mildbr. \& Burret) Mildbr. \& Burret (Harris 5241 (E)). F. D. mildbraedii Burret (Harris 4397 (E)). E-F at the same magnification (40× with microscope). Scale bars $=1 \mathrm{~mm}$. A-D images by D. Harris (RBGE); E-F images by J. Wellsow (RBGE). 
The color to which leaves dry and their texture can be useful. Leaves of $D$. subericarpa typically dry green and are papery, whereas leaves of all other species typically dry greenish-brown or brown and are sub-leathery. Fresh and dried leaves are often bullate in D. chrysochlamys.

\section{Indumentum}

The presence or absence and density of hairs are variable between species and can be useful in species delimitation, in conjunction with other characters. D. chrysochlamys is characterized by its orange pubescence and the evenly spaced, soft, stellate hairs on the lower leaf side that are easily visible using a $10 \times$ lens, and $D$. mildbraedii is characterized by small, dense, stellate hairs on the lower leaf side which can be difficult to see even with a $10 \times$ lens (see Fig. 2).

In the taxonomic descriptions, the term scattered is used when hairs on the leaf surface are distinctly separate from each other and the term dense when hairs are touching one another (or nearly so) or when they obscure the surface.

\section{Pedicels}

The character and the length of pedicels is a useful floral character for distinguishing between $D$. dewevrei (pedicels thick, 2-6 mm long) and D. mildbraedii (pedicels slender, 8-20 mm long).

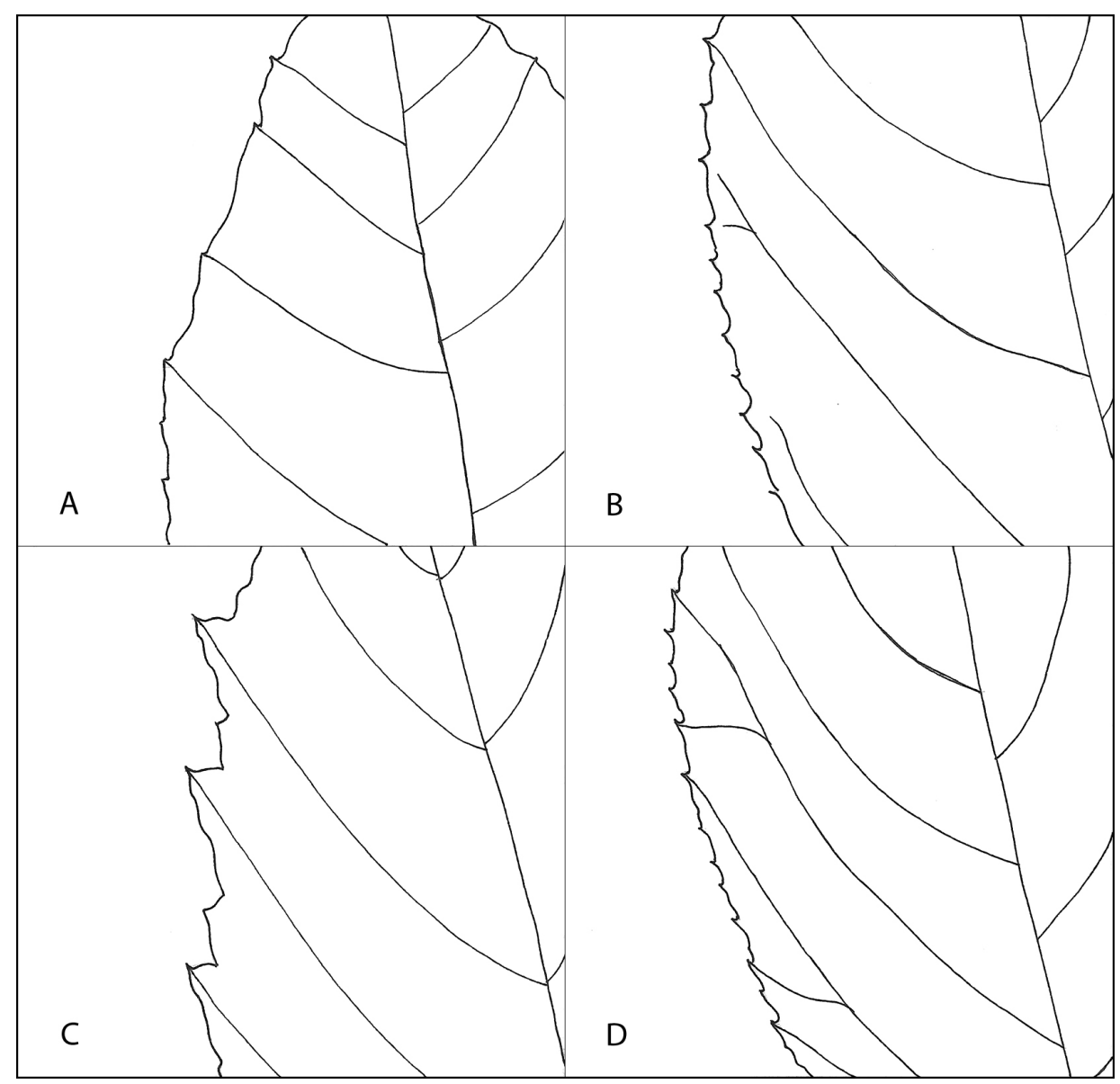

Fig. 3. Outline of Desplatsia Bocq. leaf margins. A. D. subericarpa Bocq. (Harris 1387 (E)). B. D. chrysochlamys (Mildbr. \& Burret) Mildbr. \& Burret (Harris 5402 (E)). C. D. dewevrei (De Wild. \& T.Durand) Burret (Harris 7025 (E)). D. D. mildbraedii Burret (Harris 4397 (E)). Shown at $1.5 \times$ actual size. Illustrations by Sanna Olander. 


\section{Petals}

In all Desplatsia species, the petals are much smaller than the sepals and show a glandular area at the base inside. The petal length and width clearly distinguishes $D$. dewevrei (petals oblong-roundish) from $D$. mildbraedii (petals lanceolate to obovate-oblong). However, since this character is difficult to observe in the field, petal length and width are not used in the key.

\section{Fruits}

All Desplatsia species have large, distinctive fruits (see Fig. 4) that are recorded to be dispersed by elephants and other large mammals. The 5-10 locular fruits are ellipsoid to subglobose, when mature, their size ranges from 6.5 to $25 \mathrm{~cm}$ long and from 5 to $20 \mathrm{~cm}$ wide, they are fibrous inside, smooth to slightly grooved longitudinally when fresh. Fruits of $D$. chrysochlamys are covered by orange hairs when young, soon becoming almost glabrous but with remnants of the hairs staying visible.

\section{Phenology}

Desplatsia appears not to have clear flowering or fruiting seasons based on the observations from the herbarium specimens investigated.

\section{Ecology and conservation}

Desplatsia grows in terra firma or occasionally seasonally flooded forest. The species tend to be widely distributed across West and Central Africa and many are represented in protected areas, e.g., the DzangaSangha Reserve (Harris 2002; Harris \& Wortley 2008). All species in this revision have been given a proposed conservation assessment of Least Concern (LC) based on EOO estimates from georeferenced material using GeoCAT (Bachmann et al. 2011) and an assessment of current threats to habitats across species distributions and levels of protection.

\section{Data}

Specimen data used in preparation for this revision are available from the data repository Dryad (https://doi.org/10.5061/dryad.j6q573n89) (Harris \& Wellsow 2019), from the GFBio portal (https://doi.org/10.25897/5/2war-9p81) (Wellsow \& Harris 2019) as well as the GBIF portal.

\section{Genus description}

Class Magnoliopsida Brongn.

Order Malvales Berchtold \& J.Presl

Family Malvaceae Juss., nom. cons.

Subfamily Grewioideae Hochr.

Tribe Grewieae Endl.

\section{Genus Desplatsia Bocq.}

Adansonia 7: 51 (Bocquillon 1866). - Type species: Desplatsia subericarpa Bocq.

Grewiella Kuntze, Lexicon Generum Phanerogamarum: 257 (Kuntze 1903). - Type species: Grewiella globosa (De Wild. \& T.Durand) T.Durand \& H.Durand, lectotype designated here.

Ledermannia Mildbr. \& Burret, Wissenschaftliche Ergebnisse der Deutschen Zentral-Afrika-Expedition, 1907-1908, unter Führung Adolf Friedrichs, Herzogs zu Mecklenburg. Vol. 2: Botanik: 499 (Mildbraed 1912). - Type species: L. chrysochlamys Mildb. \& Burret.

Grewiopsis De Wild. \& T.Durand Bulletin de la Société royale de Botanique de Belgique. ComptesRendus des Séances 38 (2): 176 (De Wildeman \& Durand 1899), nom. illegit., superfluous homonym, non Saporta 1865 - fossil.

Pleianthemum K.Schum. ex A.Chev. Exploration botanique de l'Afrique occidentale française. Vol. 1: Énumération des Plantes: 92 (Chevalier 1920), nom. inval., no description. 

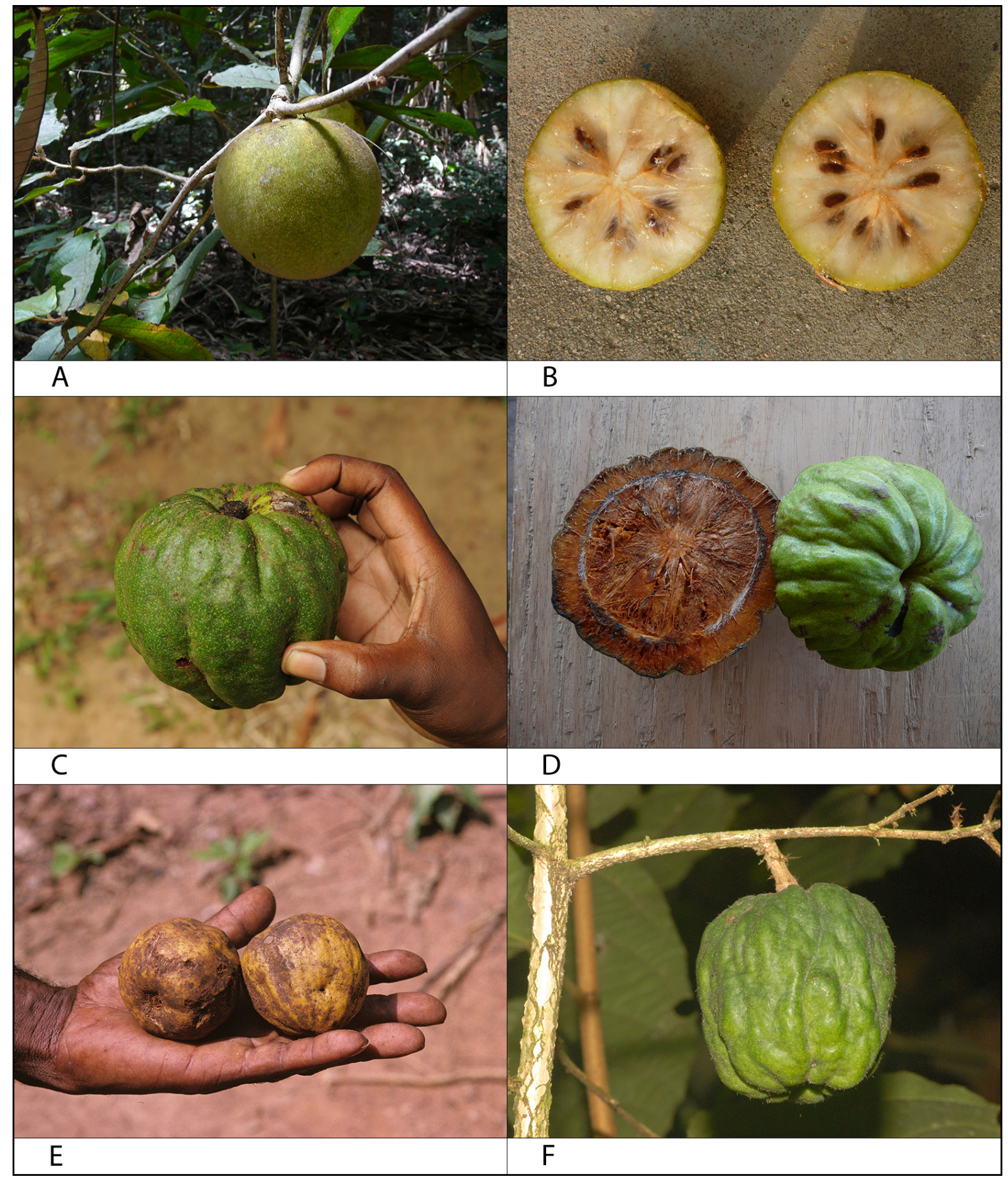

Fig. 4. Desplatsia Bocq. fruit images. A-B. D. chrysochlamys (Mildbr. \& Burret) Mildbr. \& Burret. A. Fresh fruit (Jongkind 8725). B. Cut fresh fruit (Jongkind 9717). C-D. D. dewevrei (De Wild. \& T.Durand) Burret. C. Fresh fruit (Harris 9652 (E)). D. Cut fresh fruit (Jongkind 8142). E. D. mildbraedii Burret (Harris 4005 (E)), fresh fruits. F. D. subericarpa Bocq. (Harris 10194), fresh fruit. A-B, D images by C. Jongkind; C, E-F images by D. Harris (RBGE). 


\section{Description}

HABIT. Trees or shrubs.

LeAVES. Simple, alternate, mature leaves usually 9-40 cm long, $2.8-19 \mathrm{~cm}$ wide, margins obscurely to clearly toothed; stipules subulately divided, 2-5-fid, persistent.

INFLORESCENCE. An axillary or terminal cyme of few- to many-flowered umbels with an involucre of bracts, bracts often deciduous.

FLOWERS. Pedicellate or subsessile, buds round or elliptic; sepals 5, free, boat-shaped, sometimes fringed at the apex; petals 5, much smaller than the sepals, with a glandular area at the base inside, pubescent at base; androgynophore absent; stamens numerous, joined at base forming a staminal tube with a ring of hairs; anthers subglobose, dorsifixed, dehiscent longitudinally. Ovary 5-10-locular, hairy; style with finely fringed stigma; ovules numerous, in two rows in each locule.

Fruits. Ellipsoid to subglobose, mature fruits $6.5-25 \mathrm{~cm}$ long, 5-20 cm wide, indehiscent, fibrous inside, irregularly, longitudinally 5-10 grooved, 5-10-locular.

SEEDS. Obovate, flattened, $0.7-1.8 \mathrm{~cm}$ long, $0.3-1 \mathrm{~cm}$ wide.

\section{Notes}

The genus contains four species. The fruits are mainly dispersed by elephants (Wilczek 1963; Hall \& Swaine 1981; Hawthorne 1995) and other large mammals. Desplatzia Bocq. (Chevalier 1917) is considered an orthographic variant of Desplatsia Bocq. (Bocquillon 1866).

\section{Distribution}

Tropical West and Central Africa: Sierra Leone to Angola and across to Uganda.

\section{Key to the species of Desplatsia}

1. Shrub or small tree usually $<2-3 \mathrm{~m}$ tall, $\mathrm{DBH}<10 \mathrm{~cm}$ when flowering or fruiting; mature leaves papery, usually drying green, petiole distinctly inflated in the upper part, leaf blade with distinctively acuminate leaf tip, margin obscurely toothed or with very few small, acute, forward-pointing teeth

D. subericarpa Bocq.

- Tree $>2-3 \mathrm{~m}$ tall, DBH $>10 \mathrm{~cm}$ when flowering or fruiting; mature leaves sub-leathery, usually drying greenish-brownish or brown, petiole not distinctly inflated in upper part, with acute to only slightly acuminate leaf tip, and either coarsely and irregularly toothed, sometimes with large (up to $1 \mathrm{~cm}$ ), jagged teeth, or regularly toothed with teeth generally smaller than $1 \mathrm{~cm}$

2. Twigs with dense, long, coarse, orange hairs; upper and lower side of mature leaves orange pubescent, lower side with evenly spaced, soft, stellate hairs easily visible using a $10 \times$ lens; fruits covered by orange hairs when young, soon becoming almost glabrous with remnants of hairs staying visible

D. chrysochlamys (Mildbr. \& Burret) Mildbr. \& Burret

- Twigs glabrous or almost glabrous; upper side and lower side of mature leaves glabrous or almost glabrous, or lower side covered with small, dense, stellate hairs, which are difficult to see using a $10 \times$ lens; fruits glabrous when mature

3 Tree usually $<10 \mathrm{~m}$ tall, DBH (5-)10-40 cm; mature leaves with irregular, large (up to $1 \mathrm{~cm}$ ), jagged teeth, leaves mostly glabrous below apart from some scattered single or stellate hairs, petiole almost glabrous with rusty indumentum, blade often (but not always) with domatia formed of hairs on 
underside of leaves in nerve axils; pedicels thick, 2-6 $\mathrm{mm}$ long

D. dewevrei (De Wild. \& T.Durand) Burret

- Tree usually $>10 \mathrm{~m}$ tall, DBH $40-80 \mathrm{~cm}$; mature leaves regularly toothed with teeth generally smaller than $1 \mathrm{~cm}$, leaves with very small, dense, stellate hairs below, sometimes difficult to see with a $10 \times$ lens, petiole with both short and scattered long brown hairs, no domatia; pedicels slender, $8-20 \mathrm{~mm}$ long

D. mildbraedii Burret

\section{Species descriptions}

Desplatsia chrysochlamys (Mildbr. \& Burret) Mildbr. \& Burret Figs 1B, 2A, E, 3B, 4A-B, 5

Notizblatt des botanischen Gartens und Museums zu Berlin-Dahlem 9: 819 (Burret 1926). - Ledermannia chrysochlamys Mildbr. \& Burret, Wissenschaftliche Ergebnisse der Deutschen Zentral-AfrikaExpedition, 1907-1908, unter Führung Adolf Friedrichs, Herzogs zu Mecklenburg. Vol. 2: Botanik: 499 (Mildbraed 1912). - Type: CAMEROON • Yaoundé; 352' N, $11^{\circ} 31$ E; 1897; G.A. Zenker 1406; syntype: $\mathrm{B} \uparrow$, lectotype: K, isolectotypes: BM, E[E00930373], HBG, M, P[MNHN-P-P06731541], L[L.2352858].

Desplatsia floribunda Burret, Notizblatt des botanischen Gartens und Museums zu Berlin-Dahlem 9: 819 (Burret 1926), syn. nov. - Type: CAMEROON • Moloundou; 26 Mar. 1911; G.W.J. Mildbraed 4761; syntype: $\mathrm{HBG} \bullet$ Deng Deng; $5^{\circ} 10^{\prime} \mathrm{N}, 13^{\circ} 50^{\prime} \mathrm{E}$; Apr. 1914; G.W.J. Mildbraed 8831; lectotype: $\mathrm{K}[\mathrm{K} 000241878]$, isolectotype: K[K000241879].

\section{Other material studied}

CAMEROON • Ngolebang; 10 May 1980; J.N. Asonganyi 3; P • Bitye; $3^{\circ} 01^{\prime} \mathrm{N}, 12^{\circ} 22^{\prime} \mathrm{E} ;$ G.L. Bates 711 ; $\mathrm{BM} \cdot 46 \mathrm{~km}$ NW of Bertoua, near village Ndjangané; $4^{\circ} 52^{\prime} \mathrm{N}, 1^{\circ} 23^{\prime} \mathrm{E} ; 18$ May 1967; F.J. Breteler 1357; BR[BR0000019963396], K, P, WAG[WAG.1845866] 3 sheets • Yaoundé; 352' N, 11³1' E; 26 Nov. 1960;

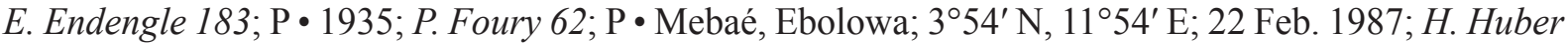
985; P• Mopanya; G. Kalbreyer 107; BM, K・ km 14 Melong-Dschang road; 5¹7' N, 959' E; 5 Jun. 1972; A.J.M. Leeuwenberg 9553; BR[BR0000019963341], K, M, P, WAG[WAG.1845857] 3 sheets • 1946; R. Letouzey 1072; P • Essam; 13 Feb. 1959; R. Letouzey 1082; P Asia; $3^{\circ} 38^{\prime}$ N, 130ㄱ' E; 21 Apr. 1961; R. Letouzey 3898; BR[BR0000019963372], K, P, WAG[WAG.1845863] 2 sheets - Nyabibete; 15 km NW of Djoum; ${ }^{\circ} 46^{\prime} \mathrm{N}, 12^{\circ} 34^{\prime} \mathrm{E}$; 22 Nov. 1966; R. Letouzey 8442; P • Bolifamba; $4^{\circ} 10^{\prime} \mathrm{N}$, $9^{\circ} 18^{\prime} \mathrm{E}$; Mar. 1929; T.D. Maitland 562; K, P• Station du Cacaoyer de N'Koemvone; $2^{\circ} 49^{\prime} \mathrm{N}, 1^{\circ} 08^{\prime} \mathrm{E}$; 26 Feb. 1975; J.J.F.E. de Wilde 8013; BR[BR0000019963358], K, P, WAG[WAG.1845855] 3 sheets • S of the Sanaga between Yaoundé and Deng Deng close to where Lom (Sanaga) and Djerem meet, about $105 \mathrm{~km}$ NE of Yaoundé; $4^{\circ} 30^{\prime} \mathrm{N}, 1^{\circ} 05^{\prime} \mathrm{E}$; Feb. 1914; G.W.J. Mildbraed 8229; K・ $1 \mathrm{~km}$ from Reserve

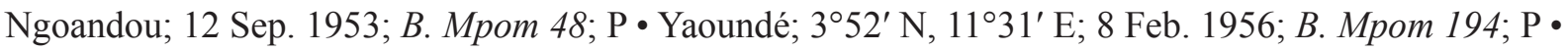
ibid.; 2 May 1959; B. Mpom 322; P • Melen, fishery station of Yaoundé; $3^{\circ} 53^{\prime} \mathrm{N}, 11^{\circ} 37^{\prime} \mathrm{E}$; 11 Oct. 1960;

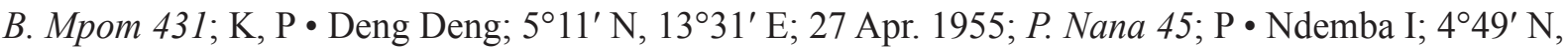
132'ㄹ E; 23 Jul.1955; P. Nana 185; P • $80 \mathrm{~km}$ from Bertoua, road to Esseleke; $4^{\circ} 48^{\prime} \mathrm{N}, 13^{\circ} 16^{\prime} \mathrm{E}$; 17 Feb. 1956; P. Nana 484; P - Forest Reserve of Metchiengoun, $14 \mathrm{~km} \mathrm{~W}$ of Bafang; $5^{\circ} 09^{\prime} \mathrm{N}, 10^{\circ} 10^{\prime} \mathrm{E}$; 17 Nov. 1982; B.A. Nkongmenek 236; P • ibid.; 18 Dec. 1981; B.A. Nkongmenek 243; P • "Réserve de faune du Dja” [Dja Faunal Reserve] 24 km S of Djolimpoum; 3²0' N, 1252' E; 27 Feb. 1994; B. Sonké 965; BR[BR0000019963327] - forest and meadows on the gently sloping side of Mount Cameroon above small Koto village; 4¹8' N, 906' E; 6 Mar. 1985; D.W. Thomas 4471; BR[BR0000019963310], K, P, WAG[WAG.1845871] • Sangha River; $2^{\circ} 22^{\prime}$ N, 1600' E; 21 Jul. 1987; D.W. Thomas 7204; K, WAG[WAG.1845729] $5 \mathrm{~km}$ SE of Mundongo, $34 \mathrm{~km}$ WNW of Muyuka; 4²4' N, 907' E; 2 Dec. 1984; J.F. Villiers 2336; P - about $5 \mathrm{~km} \mathrm{~S}$ of M'Balmayo, S of Yaoundé, on border of Nyong River; $3^{\circ} 31^{\prime} \mathrm{N}, 11^{\circ} 30^{\prime} \mathrm{E} ; 12$ Feb. 1964; W.J.J.O. de Wilde 1903; K, P, WAG[WAG.1845868] 2 sheets • Centre 
agronomique N'Kolbisson, $8 \mathrm{~km} \mathrm{~W}$ of Yaoundé; $3^{\circ} 53^{\prime} \mathrm{N}, 1^{\circ} 27^{\prime} \mathrm{E} ; 24$ May 1964; W.J.J.O. de Wilde 2622; BR[BR0000019963365], P, WAG[WAG.1845861] 2 sheets • N'Koemvone; $2^{\circ} 49^{\prime} \mathrm{N}, 1^{\circ} 08^{\prime} \mathrm{E}$;

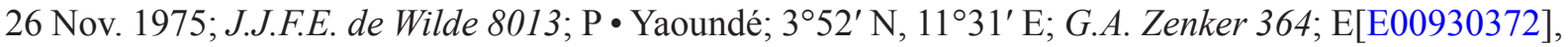
HBG.

CENTRAL AFRICAN REPUBLIC - Ndakan; $2^{\circ} 23^{\prime} \mathrm{N}, 1^{\circ} 12^{\prime} \mathrm{E} ; 16$ May 1988; D.J. Harris 680; BR[BR0000019963433], E[E00930959] • Dzanga-Sangha Reserve, Ndakan camp; $2^{\circ} 25^{\prime} \mathrm{N}, 16^{\circ} 13^{\prime} \mathrm{E}$; 19 Sep. 1988; D.J. Harris 1163; E[E00930955] - Dzanga-Sangha Reserve, Ndakan; 2²1' N, $16^{\circ} 10^{\prime}$ E; 10 Oct. 1988; D.J. Harris 1388; E[E00930953] - 25 km SE of Bayanga, Kongana research camp; $2^{\circ} 47^{\prime} \mathrm{N}, 16^{\circ} 25^{\prime} \mathrm{E} ; 2$ Feb. 1994; D.J. Harris 4433; E[E00930957] • ibid.; 21 May 1994; D.J. Harris 4908; E[E00930958] • ibid.; 247’ N, 16²5’ E; 29 May 1994; D.J. Harris 4977; E[E00930960] • SanghaMbaere, $15 \mathrm{~km}$ from Kongana on road to Bayanga, ca $15 \mathrm{~km}$ SE of Bayanga; $2^{\circ} 49^{\prime} \mathrm{N}, 16^{\circ} 19^{\prime} \mathrm{E}$; $19 \mathrm{Jan}$. 1996; D.J. Harris 5402; E[E00930948] • Boukoko; 357' N, 1755' E; 20 Sep. 1947; Tisserant (Équipe) 264; BM, P • Boukoko; 35' N, 1755' E; 20 Feb. 1951; Tisserant (Équipe) 2001; BM, P • Oubangui; C. Tisserant 129; BM, K, P• Bayanga; 254' N, 16²15' E; 20 Feb. 1976; Wraber LJU49481; K.

CONGO • Komone; $3^{\circ} 15^{\prime} \mathrm{N}, 13^{\circ} 10^{\prime} \mathrm{E}$; 24 Jan. 1968; A. Bouquet 2438; P - north side of Sombo stream, $8 \mathrm{~km} \mathrm{~N}$ of Makao; 2³9' N, 17¹0' E; 21 Apr. 1995; D.J. Harris 5241; E[E00930954] • ca 43 km NE of Kabo, Nouabalé-Ndoki National Park; $2^{\circ} 20^{\prime} \mathrm{N}, 16^{\circ} 20^{\prime} \mathrm{E}$; 6 May 2007; D.J. Harris 9117; E • ca $39.5 \mathrm{~km}$ NE of Kabo, Nouabalé-Ndoki National Park; $2^{\circ} 05^{\prime} \mathrm{N}, 16^{\circ} 21^{\prime}$ E; 22 Sep. 2013; V.P. Medjibe 742 ; E • ca $12.5 \mathrm{~km}$ NE of Kabo; ca $2^{\circ} 07^{\prime} \mathrm{N}, 16^{\circ} 10^{\prime} \mathrm{E}$; 11 Sep. 2006; G. Moukassa 142B; E • ca $16 \mathrm{~km}$ NE of Kabo; $2^{\circ} 07^{\prime} \mathrm{N}, 16^{\circ} 13^{\prime} \mathrm{E} ; 20$ Sep. 2006; G. Moukassa 239; E • ca $6.5 \mathrm{~km} \mathrm{SE}$ of Kabo; $1^{\circ} 59^{\prime} \mathrm{N}$, $16^{\circ} 05^{\prime}$ E; 13 Oct. 2006; G. Moukassa 611; E • ca 5 km E of Kabo; ca $2^{\circ} 01^{\prime} \mathrm{N}, 16^{\circ} 07^{\prime} \mathrm{E} ; 12$ Feb. 2007; G. Moukassa 2595; E・ibid.; 13 Feb. 2007; G. Moukassa 2607; E • ibid.; 14 Feb. 2007; G. Moukassa 2627; E - ca 49 km NE of Kabo, Nouabalé-Ndoki National Park; $2^{\circ} 22^{\prime} \mathrm{N}, 16^{\circ} 23^{\prime}$ E; 27 Mar. 2007; G. Moukassa 3404; E • ca 39.5 km NE of Kabo, Nouabalé-Ndoki National Park; $2^{\circ} 15^{\prime} \mathrm{N}, 16^{\circ} 22^{\prime} \mathrm{E}$; 15 Apr. 2007; G. Moukassa 3725; E[E00930956] • ca 27 km ENE of Kabo; 206’ N, 16¹9’ E; 19 May 2007; G. Moukassa 4355; E • Nouablé-Ndoki National Park, Goualougo Study Site, 37.84 km E of Bomassa; $2^{\circ} 11^{\prime} \mathrm{N}, 16^{\circ} 31^{\prime} \mathrm{E}$; 2 Dec. 2007; S.T. Ndolo Ebika 270; E[E00486065], WAG[WAG.1845835] - ca $12.5 \mathrm{~km}$ NE of Kabo; $2^{\circ} 07^{\prime}$ N, $16^{\circ} 10^{\prime}$ E; 7 Sep. 2006; F.O. Nzolani Silaho 100; E • ibid.; 8 Sep. 2006; F.O. Nzolani Silaho 147; E • ca 2 km S of Kabo; $2^{\circ} 02^{\prime}$ N, $16^{\circ} 06^{\prime}$ E; 2 Oct. 2006; F.O. Nzolani Silaho 375; E • ibid.; 4 Oct. 2006; F.O. Nzolani Silaho 434; E • ibid.; 4 Oct. 2006; F.O. Nzolani Silaho 437; E • ca $6.5 \mathrm{~km}$ SE of Kabo; $1^{\circ} 59^{\prime} \mathrm{N}, 1^{\circ} 05^{\prime}$ E; 12 Oct. 2006; F.O. Nzolani Silaho 547; E • ibid.; 13 Oct. 2006; F.O. Nzolani Silaho 569; E・ibid.; 16 Oct. 2006; F.O. Nzolani Silaho 637; E • ca 8 km ENE of Kabo; $2^{\circ} 04^{\prime}$ N, $16^{\circ} 09^{\prime}$ E; 24 Nov. 2006; F.O. Nzolani Silaho 1429; E • ca 33 km E of Kabo; $2^{\circ} 05^{\prime} \mathrm{N}, 16^{\circ} 23^{\prime} \mathrm{E}$; 8 Dec. 2006; F.O. Nzolani Silaho 1554; E - ca $29 \mathrm{~km}$ E of Kabo; $2^{\circ} 04^{\prime} \mathrm{N}, 16^{\circ} 20^{\prime} \mathrm{E}$; 8 Jan. 2007; F.O. Nzolani Silaho 1952; E - ca 43 km NE of Kabo, Nouabalé-Ndoki National Park; $2^{\circ} 20^{\prime}$ N, $16^{\circ} 20^{\prime}$ E; 3 Feb. 2007; F.O. Nzolani Silaho 2524; E • ca 5 km E of Kabo; $2^{\circ} 01^{\prime}$ N, $16^{\circ} 07^{\prime}$ E; 12 Feb. 2007; F.O. Nzolani Silaho 2596; E • ibid.; 12 Feb. 2007; F.O. Nzolani Silaho 2597; E • ibid.; 13 Feb. 2007; F.O. Nzolani Silaho 2610; E • ca 47.5 km NE of Kabo, Nouabalé-Ndoki National Park; $2^{\circ} 22^{\prime}$ N, 16²2' E; 30 Mar. 2007; F.O. Nzolani Silaho 3432; E • ca 39.5 km NE of Kabo, NouabaléNdoki National Park; $2^{\circ} 15^{\prime} \mathrm{N}, 16^{\circ} 22^{\prime}$ E; 14 Apr. 2007; F.O. Nzolani Silaho 3610; E • ca $42.5 \mathrm{~km}$ NE of Kabo, Nouabalé-Ndoki National Park; $2^{\circ} 17^{\prime}$ N, 16²2' E; 17 Apr. 2007; F.O. Nzolani Silaho 3763; E • $24 \mathrm{~km}$ ENE of Kabo; $2^{\circ} 06^{\prime} \mathrm{N}, 16^{\circ} 17^{\prime} \mathrm{E}$; 22 May 2007; F.O. Nzolani Silaho 4321; E • ca $10.5 \mathrm{~km}$ ESE of Kabo; $1^{\circ} 59^{\prime} \mathrm{N}, 16^{\circ} 09^{\prime} \mathrm{E}$; 30 May 2007; F.O. Nzolani Silaho 4562; E.

DEMOCRATIC REPUBLIC OF CONGO • Penghe; $4^{\circ} 26^{\prime}$ S, $28^{\circ} 27^{\prime}$ E; 28 Jan. 1914; J.C. Bequaert 2159; BR[BR0000019963495] 3 sheets - Walikale-Lubutu; 044' S, 26³5' E; 11 Jan. 1915; J.C. Bequaert 6567; BR[BR0000019963518] 2 sheets • Kisangani; $0^{\circ} 30^{\prime}$ N, 25¹1' E; 3 Mar. 1915; J.C. Bequaert 7021; BR[BR0000019963532] 2 sheets • Nala; 252' N, 27³9' E; 1911; A. Boone s.n.; BR[BR0000019963556] 
- Woud La Kulu; 20 Jun. 1931; J.F. van den Brande 664; BR[BR0000019964034] • ibid.; 20 Jun. 1931; J.F. van den Brande 684; BR[BR0000019964041] - Muetshi; 441' S, 22॰39' E; 7 May 1983; P. Casier 449; BR[BR0000019963464], P • Muhula, road Walikale-Lubutu; $1^{\circ} 03^{\prime} \mathrm{S}, 27^{\circ} 16^{\prime} \mathrm{E}$; 16 Nov. 1955; R.A. Christiaensen 1198; BR[BR0000019963563] - Bambesa; 3²6' N, 2541' E; H. du Bois 407; BR[BR0000019963600] - Station Inéac Bokatea; 3¹1' N, 1964' E; 3 Apr. 1955; C. Evrard 627; BR • Bambesa; 3²6' N, 2541' E; 6 Feb. 1961; P. Gérard 4827; BR[BR0000019963631] • ibid.; 16 May 1961; P. Gérard 4851; BR[BR0000019963648], K • ibid.; 24 Apr. 1962; P. Gérard 5172; BR[BR0000019963662] 2 sheets • ibid.; 13 Aug. 1962; P. Gérard 5312; BR[BR0000019963822] • DignaAngo; 8 Nov. 1963; P. Gérard 5666; BR[BR0000019963679] 2 sheets, K • Yangambi Insolo à Okamba; $0^{\circ} 46^{\prime} \mathrm{N}, 24^{\circ} 27^{\prime} \mathrm{E}$; 1948; G. Gilbert 9209; BR[BR0000019963693] - Yambata; $2^{\circ} 25^{\prime} \mathrm{N}, 2^{\circ}{ }^{\circ} 57^{\prime} \mathrm{E}$; 5 Jan. 1914; S. de Giorgi 1606; BR[BR0000019963570] 2 sheets • near Kisangani; 0³0’ N, 25¹1' E; 15 May 1921; V. Goosens 2890; BR[BR0000019963709] 3 sheets - "Station de 1'Epulu" [Epulu]; 1²5' N, 28³5' E; 28 Apr. 1981; T. Hart 46; BR[BR0000019963747] 2 sheets • Ituri Forest, Afarama; $1^{\circ} 33^{\prime} \mathrm{N}$, $28^{\circ} 32^{\prime}$ E; 6 Apr. 1993; T. Hart 1527; BR[BR0000019963730] • between Masisi and Walikale; $1^{\circ} 25^{\prime} \mathrm{N}$, 2804' E; Mar. 1932; J.P.A. Lebrun 5176; BR[BR0000019963778] 2 sheets, P • Urega, Maniema; July 1932; J.P.A. Lebrun 5704; BR[BR0000019963754] 2 sheets, P • Kisangani, Île Kongolo, at junction of Lindi with River Zaïre; 0³4' N, 2550' E; 23 Jun. 1978; J. Lejoly 3760; BR[BR0000019963792] • ibid.; 14 Dec. 1978; J. Lejoly 4408; BR[BR0000019963808] • km 30, road Kisanganito Wanié, Rukula 5 km of road; $0^{\circ} 25^{\prime} \mathrm{N}, 25^{\circ} 27^{\prime} \mathrm{E}$; 29 Jun. 1979; J. Lejoly 5253; BR[BR0000019963815] • road YabaondoYangandi, Lubiaye River; 045' N, 2354' E; 19 May 1958; A. Léonard 710; BR[BR0000019963839] - Kabunga Terr.; 24 Feb. 1958; A. Léonard 1841; BR[BR0000019963846] 2 sheets - Bengamisa; $0^{\circ} 56^{\prime} \mathrm{N}, 25^{\circ} 12^{\prime} \mathrm{E} ; 2$ May 1947; J. Léonard 1184; BR[BR0000019963877], K, WAG[WAG.1845833] - Kamisuka; 18 Aug. 1957; J. Léonard 5948; BR[BR0000019963860], K - Dembia-lbale; 3³0' N, 2550' E; 16 Apr. 1936; J. Louis 1681; BR[BR0000019963884] 2 sheets, K, P • Urega; July 1932; J. Louis 5704; P • Namoya; 400' S, 27³3' E; 12 Aug. 2008; W.R.Q. Luke 12483; K • Beni, Muera;

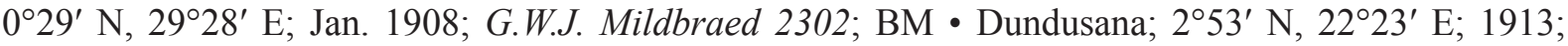
M.G. Mortehan 854; BR[BR0000019963914] 2 sheets - Kivu between Mwenda and Kalasabango; $0^{\circ} 24^{\prime} \mathrm{N}, 29^{\circ} 46^{\prime} \mathrm{E}$; 8 Sep. 1952; H.A. Osmaston 2490B; BR[BR0000019963938] • Kembe; 10 Jun. 1958; R. Pierlot 2248; BR[BR0000019963952], K • Kisangani; 0³0' N, 25¹1'; 31 Dec. 1983; L. Pauwels 6728; BR[BR0000019963945] - F. Reygaert 156; BR • Kisangani; 25 Jan. 1926; F.H.E.A.W. Robyns 1416; BR[BR0000019963983] 2 sheets, WAG[WAG.1845831] - Kalehe km 110, road KavumuWalikale, Irangi Reserve I.R.S.A.C.; 21 Jun. 1958; G. Troupin 7727; BR[BR0000019964003] 2 sheets • Yambata; 2²5' N, 215ㄱ' E; F. Vermoesen 20; BR[BR0000019964058] 2 sheets.

GABON • Oyem; 208' N, 11²1' E; G.M.P.C. Le Testu 9102; BM, BR[BR0000019963426] 2 sheets, K, P, WAG[WAG.1845837] 2 sheet.

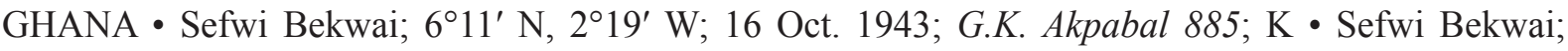
$6^{\circ} 11^{\prime} \mathrm{N}, 2^{\circ} 19^{\prime} \mathrm{W} ; 16$ Oct. 1943; G.K. Akpabal 890; K・Anibil; 459' N, $2^{\circ} 10^{\prime}$ W; J.E. Andoh 3252; BM, BR[BR0000019963297], K - Subiri Forest Reserve, Benso; Sep. 1951; J.E. Andoh FH5569; BR[BR0000019963303], K, P • Juaboso; 6²0' N, 249' W; 23 Jul. 1947; N.K. Lovi WACRI4930; K • Bia National Park; 6²3' N, $3^{\circ} 00^{\prime}$ W; 22 Nov. 1996; H.H. Schmidt 2204; BR[BR0000009037618], K, WAG[WAG.1845746] • 1941; J. Scholes 175; WAG[WAG.1845780] 3 sheets • Achimkrom, 15 miles NW of Prestea; 53' N, $2^{\circ} 15^{\prime} \mathrm{W}$; July 1928; C. Vigne 1260; BM, K.

GUINEA • Nimba Mountains, plot PERS02; 7³4' N, 8²8' W; 8 Jul. 2008; Nimba Botanic Team PD666; WAG[WAG.1845747] • "Monts Nimba" [Nimba Mountains]; Aug. 1947; R. Schnell 3429; P.

IVORY COAST • Rasso; A. Aubréville 155; BR[BR0000019963082], K, P • ibid.; 30 Oct. 1929; A. Aubréville s.n.; P • Bébasso; Feb. 1970; P. Bamps 2423; BR[BR0000019963105] 2 sheets, P • Teké; 
23 Feb. 1962; L. Bernardi 8165; P • Yapo north; 548' N, 408' W; 15 Mar. 1962; L. Bernardi 8684; K, P, WAG[WAG.1845761] • LeBanco, Abidjan; $5^{\circ} 18^{\prime}$ N, 400' E; Mar. 1951; N. Boubéé 6297; P • Yapo Forest, near Abidjan; 548' N, 408' W; 29 Jul. 1954; A.S. Boughey 14546; K 20 km SE of Aboisso; 25 Jul. 1968; F.J. Breteler 5293; BR[BR0000019963129], M, P, WAG[WAG.1845758] 2 sheets • Banco Forest Reserve, $\mathrm{N}$ of centre, near Banco River; $5^{\circ} 24^{\prime} \mathrm{N}, 4^{\circ} 03^{\prime} \mathrm{W}$; 16 Jun. 1975; W.J. van der Burg 558; WAG[WAG.1845816] 2 sheets • "Forêt de 1'Anguédédou" [Anguédédou Forest]; 5²3' N, 408' W; 25 Aug. 1975; W.J. van der Burg 783; BR[BR0000019963136], WAG[WAG.1845814] 2 sheets • A.J.B. Chevalier 11441; WAG[WAG.1845828] - Abidjan; $5^{\circ} 18^{\prime} \mathrm{N}, 4^{\circ} 00^{\prime} \mathrm{W}$; A.J.B. Chevalier 15441; BR[BR0000019963228] 2 sheets, K, P • Bouroukrou; $7^{\circ} 40^{\prime}$ N, $3^{\circ} 12^{\prime}$ W; Jan. 1907; A.J.B. Chevalier 16722; P • Bouroukrou; km 92, railway line; $7^{\circ} 40^{\prime} \mathrm{N}, 3^{\circ} 12^{\prime} \mathrm{W}$; 10 Jan. 1907; A.J.B. Chevalier 16933; BR[BR0000019963204] 2 sheets, K, P, WAG[WAG.1845827] - Bouroukrou; 740' N, 312' W; 20 Jan. 1907; A.J.B. Chevalier 16969; P • between Moyen Sassandra and Moyen Cavally; 1 Jul. 1907; A.J.B. Chevalier 19225; P • ibid.; Jul. 1907; A.J.B. Chevalier 19246; P ca 15 km NW of Sassandra on road to San Pedro; 500' N, 6¹1' W; 11 Jul. 1978; A.J.F.M. Dekker 93; WAG[WAG.1845796] 2 sheets - "forêt de Banco" [Banco Forest], ca $2 \mathrm{~km} \mathrm{~W}$ of Abidjan, beside the road in direction of Route des Aviateurs, ca $2 \mathrm{~km}$ before bridge over the Banco River; 5⒉ $3^{\prime} \mathrm{N}, 4^{\circ} 03^{\prime} \mathrm{W}$; 1 Aug. 1978; A.J.F.M. Dekker 107; WAG[WAG.1845797] • pass near Dabou;Aug. 1958; C. Farron s.n.; WAG[WAG.1845763] 2 sheets • "Forêt d'Yapo"[Yapo Forest]; 9 Oct. 1957; C. Farron s.n.; WAG[WAG. 1845767] 2 sheets • E of Dakpadou; $5^{\circ} 16^{\prime} \mathrm{N}, 6^{\circ} 00^{\prime} \mathrm{W} ; 24$ Aug. 1967; C. Geerling 776; BR[BR0000019963167], K, WAG[WAG.1845764] 2 sheets $\bullet$ Banco Forest Reserve, North-west part, near Anguédédou Forest; $5^{\circ} 25^{\prime}$ N, $4^{\circ} 04^{\prime}$ W; 28 Jul. 1973; J. de Koning 2028; WAG[WAG. 1845820] 2 sheets • Anguédédou Forest; $5^{\circ} 23^{\prime}$ N, $4^{\circ} 08^{\prime}$ W; 28 Aug. 1973; J. de Koning 2190; BR[BR0000019963211], WAG[WAG.1845824] 2 sheets • Banco Forest Reserve, South-central part; $5^{\circ} 22^{\prime} \mathrm{N}, 4^{\circ} 03^{\prime} \mathrm{W}$; 8 Nov. 1973; J. de Koning 2612; BR[BR0000019963181], WAG[WAG.1845818] 2 sheets • along Agnéby River, $30 \mathrm{~km}$ new road Abidjan-Ndouci; $5^{\circ} 30^{\prime} \mathrm{N}, 4^{\circ} 15^{\prime} \mathrm{W}$; 28 May 1979; A.P.M. de Kruif 74; BR[BR0000019963075], WAG[WAG.1845798] • Yapo Forest, $40 \mathrm{~km}$ $\mathrm{N}$ of Abujan; $5^{\circ} 48^{\prime} \mathrm{N}, 4^{\circ} 08^{\prime} \mathrm{W}$; 31 Oct. 1958; A.J.M. Leeuwenberg 1849; BR[BR0000019963150], K, P, WAG[WAG.1845809] 2 sheets • km 44 Abidjan-Adzope road; 5³7' N, 403' W; 27 Jul. 1970; A.J.M. Leeuwenberg 7961; BR[BR0000019963198], K, L[L.4216445], M, P, WAG[WAG.1845822] 3 sheets $\bullet$ Banco; 5 ${ }^{\circ} 21^{\prime}$ N, 403' W; 15 Jan. 1931; Martineau 307; BR[BR0000019963099], K, P • Lobykro; 742' N, 650' W; 22 Aug. 2001; L. Nusbaumer LN539; P, WAG[WAG.1845802] • Lobykro; $7^{\circ} 42^{\prime}$ N, $6^{\circ} 50^{\prime}$ W; 10 Aug. 2001; L. Nusbaumer LN745; P • "forêt du Banco" [Banco Forest], ca 3 km NW of Abidjan; 4 Jul. 1963; R.A.A. Oldeman 118; BR[BR0000019963143], WAG[WAG.1845845] • Mbaso, E of Adzopé; 606' N, 353' W; 26 Jul. 1963; R.A.A. Oldeman 198; BR[BR0000019963280], K, P, WAG[WAG.1845777] 2 sheets • Banco Forest; 5²1' N, 403' W; 29 Jul. 1954; R. Schnell 5830; P • Guiflo-Tai; Aug. 1954; R. Schnell 6047; P • Abidjan, "forêt d'Anguédedou" [Anguédédou Forest]; $5^{\circ} 24^{\prime} \mathrm{N}, 4^{\circ} 07^{\prime} \mathrm{W}$; 5 Sep. 1969; M.T. Thijssen 305; BR[BR0000019963068], K, WAG[WAG.1845778] 2 sheets $\bullet$ San Pedro; $4^{\circ} 44^{\prime}$ N, $6^{\circ} 38^{\prime}$ W; 3 Aug. 1901; Thoiré 316; K, P • surroundings of Akoupé, $30 \mathrm{~km}$ NW of Abidjan; $5^{\circ} 28^{\prime} \mathrm{N}, 3^{\circ} 48^{\prime} \mathrm{W} ; 22$ May 1969; C. Versteegh 114; U[U.1380068], WAG[WAG.1845844] 3 sheets; • Yapo Forest; ca $5^{\circ} 41^{\prime} \mathrm{N}, 4^{\circ} 05^{\prime} \mathrm{W}$; 30 Aug. 2001; J.J. Wieringa 4259; WAG[WAG.1845801] 3 sheets • Adiopodoumé, "forêt d'O.R.S.T.O.M." [forest of O.R.S.T.O.M.]; 7 Jul. 1956; J.J.F.E. de Wilde 77; WAG[WAG.1845774] 3 sheets $17 \mathrm{~km} \mathrm{~W}$ of Abidjan, Idert Forest; $5^{\circ} 18^{\prime} \mathrm{N}, 4^{\circ} 00^{\prime} \mathrm{W}$; $12 \mathrm{Jul}$. 1963; W.J.O. de Wilde 449; BR[BR0000019963273], K, P, WAG[WAG.1845769] 3 sheets 3 km NW of Adiopodoumé; 5²0' N, 407’ W; 23 Jul. 1963; W.J.O. de Wilde 516; BR[BR0000019963266], K, P, WAG[WAG.1845810] 3 sheets - Nigbi II, $11 \mathrm{~km}$ SSW of Soubré W of the Sassandra River; 18 Nov. 1961; J.J.F.E. de Wilde 3253; WAG[WAG.1845841] 3 sheets • "Forêt de Tebe" [Tebe Forest]; 23 Aug. 1955; H.C.D. de Wit 8014; WAG[WAG.1845806] 2 sheets • Banco, "Forêt classée" [forest]; 16 Jan. 1961; H.C.D. de Wit 9058; WAG[WAG.1845847] 2 sheets.

LIBERIA • Zokatown; 28 Nov. 1958; J.G. Adam 16206; P • Nimba; 7²9' N, 8³4' W; 20 Sep. 1971; J.G. Adam 26117; K, P • Webo district, Diebla; 7³1' N, 8³5’ W; 2 Jul. 1947; J.T. Baldwin 6367; 
K - Tchien-along the road from Zwedru to Sinoe; $6^{\circ} 00^{\prime}$ N, $8^{\circ} 10^{\prime}$ W; 23 Jul. 1969; F. Blyden 928; BR[BR0000019963006], WAG[WAG.1845783] 2 sheets • Grand Gedeh county, near Tobli, Sica road; 6¹9' N, 8³3' W; 20 Jan. 1967; J.J. Bos 2925; WAG[WAG.1845791] 4 sheets • 1936; W.J. Harley 995; $\mathrm{K} \bullet 10$ miles SE of Kakata, Firestone division 33; 6²4' N, 10¹8' W; 21 Jun. 1970; J.W.A. Jansen 1945; BR[BR0000019963044], WAG[WAG.1845756] 2 sheets 10 miles NW of Tchien, Fijnhout exploitation; $6^{\circ} 11^{\prime} \mathrm{N}, 8^{\circ} 11^{\prime} \mathrm{W} ; 22$ Jul. 1970; J.W.A. Jansen 2097; BR[BR0000019963051], WAG[WAG.1845754] 2 sheets $\bullet$ Kakata; $6^{\circ} 36^{\prime}$ N, $10^{\circ} 28^{\prime}$ W; Aug. 1970; J.W.A. Jansen 2186 ; P • Cestos-Sanguin area, Logging Consession of the Cooper's; $5^{\circ} 32^{\prime} \mathrm{N}, 9^{\circ} 23^{\prime} \mathrm{W} ; 7$ Dec. 2002; C.C.H. Jongkind 5699; WAG[WAG.1845743] 2 sheets • east slope of the Putu Hills East Range W of Tiama Town; 5³9' N, $8^{\circ} 09^{\prime}$ W; 20 May 2005; C.C.H. Jongkind 6250; BR[BR0000019963037], WAG[WAG.1845751] • between Geeblo Town and top of Tiele Hill; $5^{\circ} 36^{\prime} \mathrm{N}, 8^{\circ} 11^{\prime} \mathrm{W}$; 4 Jun. 2005; C.C.H. Jongkind 6603; WAG[WAG.1845760] • North Lorma National Forest; $8^{\circ} 02^{\prime}$ N, 944' W; 21 Nov. 2005; C.C.H. Jongkind 6791; WAG[WAG.1845749] - Grebo Forest; 5²4' N, 7²4' W; 9 Dec. 2005; C.C.H. Jongkind 7249; WAG[WAG.1845750]・ Inside Sapo NP close to cano crossing of Sinoe River; $5^{\circ} 20^{\prime}$ N, $8^{\circ} 47^{\prime}$ W; 7 Mar. 2009; C.C.H. Jongkind 8873; WAG[WAG.1845741] • Putu Hills, East Range; $5^{\circ} 38^{\prime}$ N, $8^{\circ} 10^{\prime}$ W; 25 Jan. 2010; C.C.H. Jongkind 9234; BR[BR0000019962993], WAG[WAG.1845748] - SW of Togba Ville; 5²8' N, $9^{\circ} 16^{\prime}$ W; 2 Dec. 2010; C.C.H. Jongkind 10019; WAG[WAG.1845745] - Sino, E of Greenville-Zwedu road; 5 ${ }^{\circ} 16^{\prime} \mathrm{N}, 8^{\circ} 57^{\prime} \mathrm{W}$; 23 Sep. 2013; C.C.H. Jongkind 12105; BR[BR0000019963020] - Sino, ca $50 \mathrm{~km}$ E of Greenville; $5^{\circ} 04^{\prime} \mathrm{N}, 8^{\circ} 32^{\prime} \mathrm{W} ; 22$ Mar. 2014; C.C.H. Jongkind 12615; BR[BR0000019963013] 2 sheets • along "Babooni Road"; 5³0' N, 8³7' W; 30 Jan. 2016; C.C.H. Jongkind 12870; BR[BR0000015223876V], WAG[WAG.1943852] - Division 16, Firestone Plantations, Harbel; 6 6 $24^{\prime}$ N, $10^{\circ} 26^{\prime}$ W; 11 Sep. 1965; J. Kokulo 9; WAG[WAG.1845789] 2 sheets - Gbnaga; 26 Sep. 1926; D.H. Linder 781; K, WAG[WAG.1845785] 2 sheets.

SIERRA LEONE • Levuma (Koya); $8^{\circ} 10^{\prime} \mathrm{N}, 10^{\circ} 28^{\prime} \mathrm{W} ; 12$ Dec. 1939; F.C. Deighton 3850; K • Gola National Park; $7^{\circ} 15^{\prime}$ N, $11^{\circ} 18^{\prime}$ W; 25 Oct. 2013; B. Saradugu 32; K, WAG[WAG.1962832] 2 sheets.

UGANDA • near Busingiro, Budongo Forest; $1^{\circ} 43^{\prime}$ N, 31 ${ }^{\circ} 28^{\prime}$ E; 8 Apr. 1950; H.C. Dawkins 557; BM,

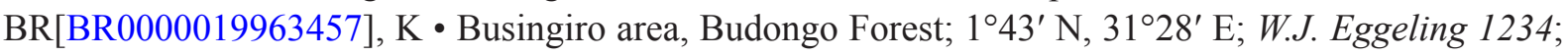
$\mathrm{K} \cdot$ slopes of Ruwenzori down to Bwamba; Dec. 1924; T.D. Maitland 1059; K • Kabango, Bwamba; $0^{\circ} 46^{\prime} \mathrm{N}, 30^{\circ} 7^{\prime} \mathrm{E}$; 22 Nov. 1935; A.S. Thomas 1517; BR[BR0000019963440], K.

\section{Description}

HABIT. Tree, to 4-8(-12) $\mathrm{m}$ tall, DBH to (6-)10-25 cm; branches and twigs with dense, long, coarse, orange hairs.

LeAves. With (2-)3-4(-5)-fid stipules, almost divided to base into linear to lanceolate lobes with acute apex, (3-)6-15 mm long, 1-5 mm wide at base, rusty-tomentose; petioles rusty-velvety, $1-3.2(-4.5) \mathrm{cm}$ long; leaf blades usually drying brown, oblong-oblanceolate to oblong-elliptic, sub-leathery, often bullate, 9-40 cm long, 3.5-19 cm wide, orange-pubescent above and below, below more so with evenly but not densely spaced stellate hairs easily visible using a $10 \times$ lens, feeling soft when touched; midrib prominent and densely orange-pubescent below, secondary veins rarely looping, apex acute or only slightly acuminate, base cordate or subcordate and sometimes slightly asymmetrical, margins coarsely and irregularly toothed, particularly in upper part of the leaf; domatia absent.

INFLORESCENCE. An axillary or terminal cyme of 3-6 flowers, usually condensed; peduncle up to $1.5 \mathrm{~cm}$ long, pubescent; involucral bracts 6-8, 3 inner roundish, about up to $8 \mathrm{~mm}$ in diameter, silky tomentose, enveloping the buds, occasionally falling off early during anthesis; 3-5 outer oblong-lanceolate, orange tomentose, not enveloping the buds, falling off early during anthesis. 
FLOWERS. Subsessile or shortly pedicellate, occasionally pedicels up to $1 \mathrm{~cm}$ long, orange-silky tomentose; buds roundish, silky-orange tomentose; sepals greenish-white or pinkish, oblong, $0.8-1.7 \mathrm{~cm}$ long, 3-4 mm wide, fringed at the tip, tomentose; petals ovate-oblong, $4 \mathrm{~mm}$ long, $1.5 \mathrm{~mm}-2.4 \mathrm{~mm}$ wide, curled back at top; ovary 5-7-locular.

Fruits. Subglobose to globose, (5-)8-12 cm long, (4-)8-9 $\mathrm{cm}$ wide, smooth to obscurely grooved, when young with orange hairs, soon almost glabrous, with visible remnants of hairs.

SEEDS. Obovoid, $1 \mathrm{~cm}$ long, $5 \mathrm{~mm}$ wide.

\section{Distribution (see Fig. 5)}

Cameroon, Central African Republic, Congo, Democratic Republic of Congo, Gabon, Ghana, Guinea, Ivory Coast, Liberia, Sierra Leone, Uganda.

\section{Habitat}

Terra firma and seasonally flooded forest, often in light gaps and open places. At altitudes of 100 $1250 \mathrm{~m}$ a.s.1. In Ghana D. chrysochlamys is reported (W. Hawthorne pers. comm.) to be commoner in the higher rainfall evergreen forest than in the lower rainfall semi-evergreen and semi-deciduous forests.

\section{Phenology}

Flower: February-May, September, December; fruit: July, September, December.

\section{Proposed IUCN status}

Least Concern (LC). Desplatsia chrysochlamys has an EOO of $3916983 \mathrm{~km}^{2}$ and an AOO of $504 \mathrm{~km}^{2}$, with a wide distribution across the forests of West and Central Africa. There are no major threats. Several collections are recorded from protected areas which give this species some level of protection.

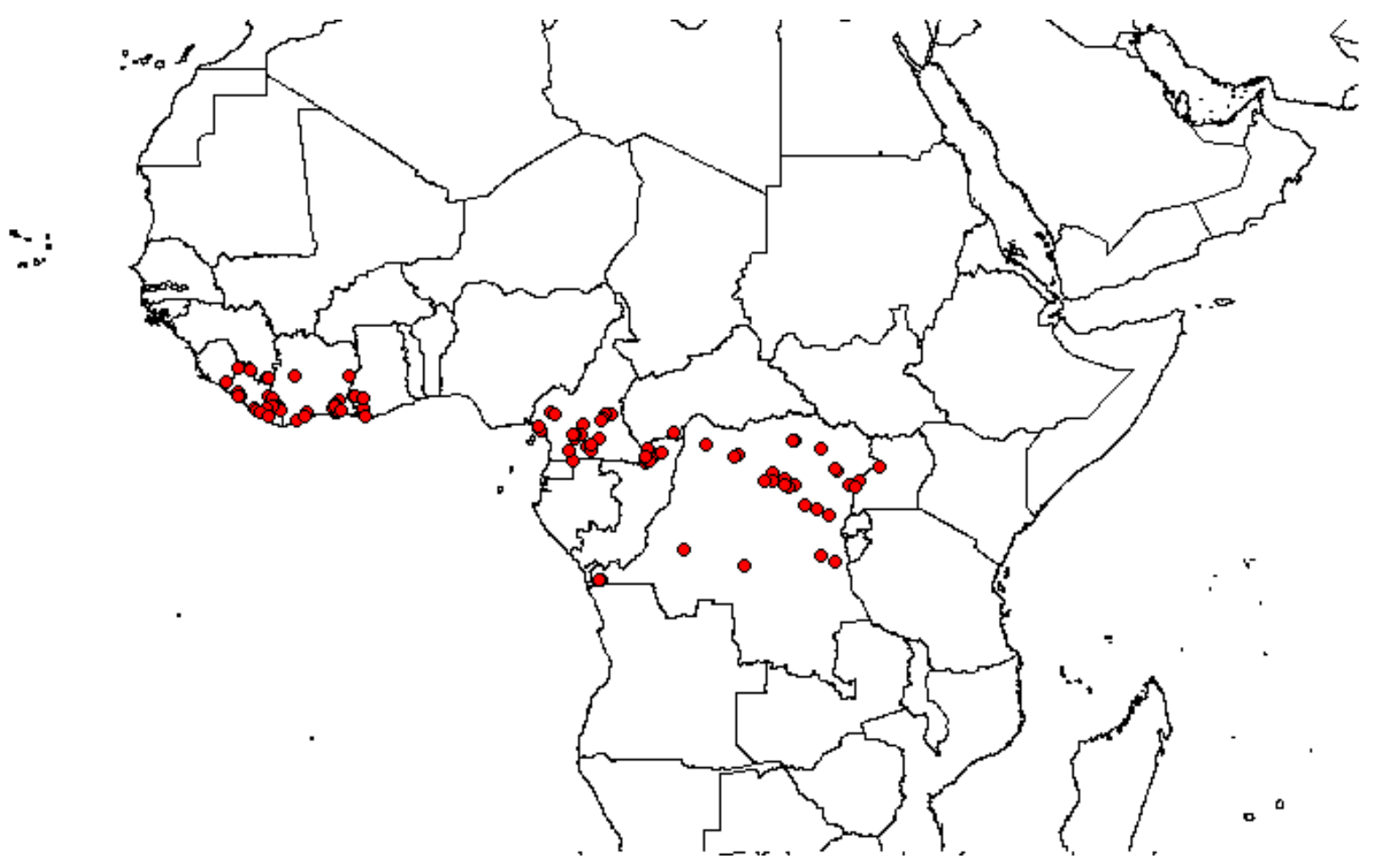

Fig. 5. Distribution map for Desplatsia chrysochlamys (Mildbr. \& Burret) Mildbr. \& Burret. 


\section{Notes}

Harris (2002) observed that some specimens identified as D. chrysochlamys from the Dzanga-Sangha area in the south west of the Central African (e.g., Wraber 49481 (K)) have expanded inflorescences and flowers with obvious pedicels and no involucral bracts, which makes the specimen very similar to Mildbraed 8831 (K) from Deng Deng (Cameroon), which is one of the two syntypes mentioned in the protologue of D. floribunda syn. nov. (Burret 1926). Since the vegetative characters fit those of D. chrysochlamys and there is some variation in the degree of inflorescence expansion, we have treated $D$. floribunda syn. nov. as a synonym of $D$. chrysochlamys. This view is further supported by the here newly defined key characters for D. chrysochlamys, of the leaves drying brown and the lower side of the leaves feeling soft when touched, both characters which are already mentioned in the protologue of D. floribunda syn. nov. (Burret 1926).

Desplatsia dewevrei (De Wild. \& T.Durand) Burret

Figs 1C, 2B, 3C, 4C-D, 6A-B, 7, Table 1

Wissenschaftliche Ergebnisse der Deutschen Zentral-Afrika-Expedition, 1907-1908, unter Führung Adolf Friedrichs, Herzogs zu Mecklenburg. Vol. 2: Botanik: 496 (Mildbraed 1912). - Grewiopsis dewevrei De Wild. \& T.Durand, Bulletin de la Société royale de Botanique de Belgique, ComptesRendus des Séances 38 (2): 177 (De Wildeman \& Durand 1899). - Grewiella dewevrei (De Wild. \& T.Durand) T. \& H.Durand, Sylloge Florae Congolanae: 70 (Durand \& Durand 1909). - Type: DEMOCRATIC REPUBLIC OF CONGO - Bumba; 5 Jun. 1896; A.P. Dewèvre 901; lectotype: BR[BR0000008965905], designated here, isolectotype: BR[BR0000008964618].

Desplatsia dewevrei var. subintegrifolia (De Wild. \& T.Durand) Burret, Wissenschaftliche Ergebnisse der Deutschen Zentral-Africa-Expedition, 1907-1908, unter Führung Adolf Friedrichs, Herzogs zu Mecklenburg. Vol. 2: Botanik: 496 (Mildbraed 1912). - Grewiopsis dewevrei var. subintegrifolia De Wild. \& T.Durand, Bulletin de la Société royale de Botanique de Belgique, Comptes-Rendus des Séances 38 (2): 178 (De Wildeman \& Durand 1899). - Grewiella dewevrei var. subintegrifolia (De Wild. \& T.Durand) T.Durand \& H.Durand, Sylloge Florae Congolanae: 70 (Durand \& Durand 1909). - Type: DEMOCRATIC REPUBLIC OF CONGO • Bokakata; 1896; A.P. Dewèvre 803; holotype: BR[BR0000008965578].

Grewiopsis trillesiana Pierre ex De Wild., Annales du Musée du Congo belge. Série 5 Botanique. Vol. 1: Etudes de systématique et de géographie botanique sur la Flore du Bas-et du MoyenCongo: 57 (De Wildeman 1903), syn. nov. - Desplatsia trillesiana (Pierre ex De Wild.) Pierre ex A.Chev., Les végétaux utiles de l'Afrique tropicale française. Etudes scientifiques et agronomiques. Vol. 9: 81 (Chevalier 1917), syn. nov. - Type: GABON - Bota; Sep. 1899; R.P.H. Trilles 189; lectotype: P[MNHN-P-P06610888], designated here, isolectotypes: BR[BR0000020060992], $\mathrm{P}[\mathrm{MNHN}-\mathrm{P}-\mathrm{P} 06610887]$.

Desplatsia lutea A.Chev. ex Hutch. \& Dalziel, Flora of West Tropical Africa 1: 240 (Hutchinson \& Dalziel 1927). - Type: IVORY COAST • Pays de Dyolas; 26 Apr. 1909; A.J.B. Chevalier 21323; lectotype: $\mathrm{K}[\mathrm{K} 000241881]$, designated here, isolectotypes: K[K000241882], P[MNHN-P-P06610828] 2 sheets, WAG[WAG.1845668]. - NIGERIA - Ibadan Forest Reserve; 17 Nov. 1900; Punch 50; syntype: K[K000241880].

Duboscia acuminata A.Chev., Bulletin de la Société botanique de France 58 (Mémoire 8d): 139 (Chevalier 1912). - Type: IVORY COAST - Zaranon; A.J.B. Chevalier 16272; lectotype: $\mathrm{P}[\mathrm{MNHN}-\mathrm{P}-\mathrm{P} 00375121]$, designated here, isolectotype: $\mathrm{P}[\mathrm{MNHN}-\mathrm{P}-\mathrm{P} 00375122]$.

\section{Other material studied}

ANGOLA • Mayumbe; 1916; J. Gossweiler 6183; BM • Cabinda; 5³3’ S, 12¹2’ E; J. Gossweiler 6640; $\mathrm{BM}, \mathrm{K}$. 
CAMEROON • Apr. 1895; G.L. Bates 164; K・Bitye, Yaoundé; $3^{\circ} 01^{\prime} \mathrm{N}, 12^{\circ}$ E; G.L. Bates 919; BM • $1 \mathrm{~km}$ above Gr. Batanga ferry; 252' N, 9`54' E; 24 Jan. 1969; J.J. Bos 3761; P • Bertoua, 5 km along road to Batouri, before junction of road to Bétaré Oya; $4^{\circ} 35^{\prime} \mathrm{N}, 13^{\circ} 44^{\prime} \mathrm{E} ; 29$ Jul. 1961; F.J. Breteler 1707; WAG[WAG.1845714] 2 sheets • Ndanan; $23^{\circ} 37^{\prime} \mathrm{N}, 1^{\circ} 34^{\prime} \mathrm{N}$; 15 Oct. 2002; M.R. Cheek 11113; BR[BR0000019964195], K, WAG[WAG.1845732] • Djoum; 2³5' N, 1249' E; 5 Dec. 2014; M.R. Cheek 17802; K・Likomba Bush, Tiko; 17 Nov. 1971; S.N. Ekema E41; WAG[WAG.1845740] 2 sheets •1935; Foury 96; P • Djembe road head; $2^{\circ} 12^{\prime} \mathrm{N}, 16^{\circ} 05^{\prime} \mathrm{E}$; 17 Oct. 1998; D.J. Harris 5954; E[E00930969] - Small Bai; $2^{\circ} 19^{\prime} \mathrm{N}, 15^{\circ} 46^{\prime} \mathrm{E}$; 31 Oct. 1998; D.J. Harris 6096; E • Yokadouma; $3^{\circ} 15^{\prime} \mathrm{N}, 1^{\circ} 03^{\prime} \mathrm{E}$; 8 Apr. 1927; S.A. Hedin 616; P • near Sanaga, road from Bafia to Yaoundé; 352' N, $11^{\circ} 31^{\prime} \mathrm{E}$; 1928 ; S.A. Hedin 1925; P • Mdiki; Nov. 1938; H. Jacques-Félix 2509; K, P, WAG[WAG.1845735] 2 sheets •

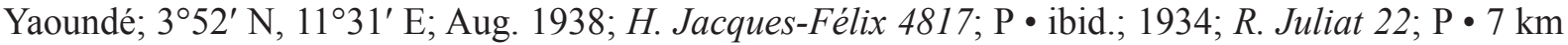
W of Yokadouma; 331' N, 1503' E; 22 Jul. 1965; A.J.M. Leeuwenberg 6232; BR[BR0000019964157], K, WAG[WAG.1845702] 4 sheets - Mungo River, near bridge of road Loum-Kumba; 8 Oct. 1965; A.J.M. Leeuwenberg 6844; BR[BR0000019964218], P, WAG[WAG.1845671] • km 14 MelongDschang road; 5'17' N, 9'59' E; 19 Jan. 1972; A.J.M. Leeuwenberg 9220; BR[BR0000019964188], WAG[WAG.1845721] 2 sheets • near Meuban, $24 \mathrm{~km} \mathrm{~S}$ of Djoum; $2^{\circ} 27^{\prime} \mathrm{N}, 12^{\circ} 40^{\prime} \mathrm{E} ; 13$ Nov. 1966; R. Letouzey 8343; P • Yaoundé; $3^{\circ} 52^{\prime} \mathrm{N}, 1^{\circ}{ }^{\circ} 1^{\prime}$ E; 30 Apr. 1959; B. Mpom 321; P・Dimako; $4^{\circ} 22^{\prime} \mathrm{N}$, 13³4' E; 19 Apr. 1955; P. Nana 24; P • Deng Deng; 17 Dec. 1955; P. Nana 384; P • about 7 km NE

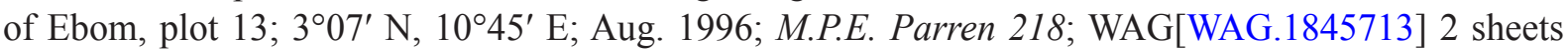

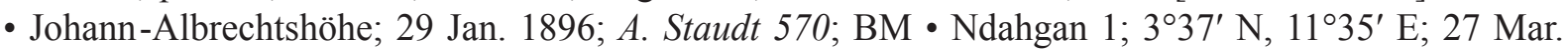
2004; F. Tadjouteu 591; K, WAG[WAG.1845731] • Southern Bakundu Forest Reserve; 4³5’ N, 9²3' E; 10 Feb. 1986; D.W. Thomas 5553; BR[BR0000019964171], K, WAG[WAG.1845725] • ca $50 \mathrm{~km} \mathrm{~S}$ of Badjob, SW of Eséka; 341' N, 1041' E; W.J.O. de Wilde 1741B; BR[BR0000019964232], K, P, WAG[WAG.1845708] 3 sheets - ca $40 \mathrm{~km} \mathrm{~S}$ of Badjob, ca $50 \mathrm{~km} \mathrm{SW}$ of Eséka near the Nyong River; $3^{\circ} 40^{\prime} \mathrm{N}, 10^{\circ} 40^{\prime} \mathrm{E}$; 18 Dec. 1963; W.J.J.O. de Wilde 1541; P, WAG[WAG.1845717] • ca $50 \mathrm{~km}$ $\mathrm{S}$ of Badjob, SW of Eséka; 341' N, 1041' E; W.J.O. de Wilde 1741A; BR[BR0000019964249], K, P, WAG[WAG.1845705] 2 sheets • Bipinde; $3^{\circ} 05^{\prime} \mathrm{N}, 10^{\circ} 25^{\prime} \mathrm{E}$; 1896; G.A. Zenker 924; BM, E[E00930370] 2 sheets, K, M, P.

CENTRAL AFRICAN REPUBLIC • Dzanga-Sangha Reserve $20 \mathrm{~km}$ E of Bayanga; 2 ${ }^{\circ} 51^{\prime} \mathrm{N}, 16^{\circ} 28^{\prime} \mathrm{E}$; 17 Nov. 1998; R.W. Carroll 1567; E[E00930962] - J.M. Fay 8402; MO - J.M. Fay 8414; MO • A.H. Gentry 62581; E • A.H. Gentry 62606; E • $2^{\circ} 21^{\prime}$ N, $16^{\circ} 09^{\prime}$ E; 12 Apr. 1988; D.J. Harris 487; E - Botembi; 2 Dec. 1959; M. Guigonis 1871; P - Dzanga-Sangha Reserve, Ndakan; $2^{\circ} 22^{\prime}$ N, $16^{\circ} 09^{\prime}$ E; 3 Sep. 1988; D.J. Harris 1036; E[E00930964] - Kongana research camp, $25 \mathrm{~km}$ SE of Bayanga; $2^{\circ} 47^{\prime} \mathrm{N}, 16^{\circ} 16^{\prime} \mathrm{E}$; 31 Jan. 1994; D.J. Harris 4368; E[E00930965] • ibid.; 1 Feb. 1994; D.J. Harris 4395; E[E00930967] • Sangha-Mbaere, Kongana camp; 247’ N, 16²6' E; 8 Mar. 1996; D.J. Harris 5496; E[E00930966] - Kongana camp, $25 \mathrm{~km} \mathrm{SE}$ of Bayanga; 2 ${ }^{\circ} 47^{\prime} \mathrm{N}, 16^{\circ} 26^{\prime} \mathrm{E} ; 4$ Nov. 2000; D.J. Harris 7025; E[E00930951] • ibid.; 5 Nov. 2000; D.J. Harris 7048; E • ibid.; 9 Nov. 2000; D.J. Harris 7136; E • ibid.; 9 Nov. 2000; D.J. Harris 7144; E[E00930961] • Elembe ya Ngombe plot, W of Sangha River; $2^{\circ} 58^{\prime} \mathrm{N}, 16^{\circ} 11^{\prime}$ E; 1 Dec. 2000; D.J. Harris 7237; E - Madibwé, close to St. Francois road; ca $12 \mathrm{~km} \mathrm{NE}$ of Bayanga, $2^{\circ} 58^{\prime} \mathrm{N}, 1^{\circ} 18^{\prime} \mathrm{E}$; 2 May 2001; D.J. Harris 7502; E • ibid.; 4 May 2001; D.J. Harris 7550; E[E00930970] - Boukoko; 354' N, 1755' E; 2 Sep. 1947; C. Tisserant 185; BM, K, P, WAG[WAG.1845738].

CONGO • village of Ouesso-Mbila; $1^{\circ} 13^{\prime} \mathrm{N}, 15^{\circ} 47^{\prime} \mathrm{E}$; 17 Jul. 1965; A. Bouquet 1574; P • Ouesso; $1^{\circ} 36^{\prime} \mathrm{N}, 16^{\circ} 03^{\prime} \mathrm{E}$; F.G. Grison 102 RG 907; P - north side of Sombo stream, 8 km N of Makao; $2^{\circ} 39^{\prime} \mathrm{N}$, 17¹0’ E; 1 May 1995; D.J. Harris 5298; E[E00930968] • Nouabalé-Ndoki National Park, Goualougo study site, $38 \mathrm{~km} \mathrm{E}$ of Bomassa; $2^{\circ} 10^{\prime} \mathrm{N}, 16^{\circ} 31^{\prime} \mathrm{E}$; 11 Jun. 2002; D.J. Harris 7975; E • ca $31 \mathrm{~km} \mathrm{E}$ of Kabo; $2^{\circ} 05^{\prime} \mathrm{N}, 16^{\circ} 21^{\prime} \mathrm{E}$; 19 Feb. 2007; D.J. Harris 8901; E - ca $41 \mathrm{~km}$ NE of Kabo, NouabaléNdoki National Park; 2²0' N, $16^{\circ} 19^{\prime}$ E; 7 May 2007; D.J. Harris 9133; E • ca 39.5 km NE of Kabo, 
Nouabalé-Ndoki National Park; 2¹5’ N, 16²2' E; 8 May 2007; D.J. Harris 9149; E • ca 6.5 km SE of Kabo; $1^{\circ} 59^{\prime} \mathrm{N}, 16^{\circ} 05^{\prime} \mathrm{E}$; 17 Jun. 2007; D.J. Harris 9280; E • ibid.; 17 Jun. 2007; D.J. Harris 9282; $\mathrm{E} \bullet$ ca $18 \mathrm{~km}$ ESE of Kabo; $1^{\circ} 59^{\prime} \mathrm{N}, 1^{\circ} 14^{\prime} \mathrm{E}$; 19 Jun. 2007; D.J. Harris 9326; E • ca $10.5 \mathrm{~km}$ ESE of Kabo; $1^{\circ} 59^{\prime} \mathrm{N}, 16^{\circ} 09^{\prime} \mathrm{E}$; 21 Jun. 2007; D.J. Harris 9368; E • ca $5 \mathrm{~km}$ E of Kabo; $2^{\circ} 01^{\prime} \mathrm{N}, 1^{\circ} 07^{\prime} \mathrm{E}$; 22 Jun. 2007; D.J. Harris 9376; E • ibid.; 22 Jun. 2007; D.J. Harris 9388; E • ca 2 km S of Kabo; $2^{\circ} 02^{\prime} \mathrm{N}, 16^{\circ} 06^{\prime} \mathrm{E}$; 23 Jun. 2007; D.J. Harris 9399; E • ca $3 \mathrm{~km} \mathrm{NE}$ of Bomassa, ca $1 \mathrm{~km} \mathrm{NE}$ of Bon Coin; $2^{\circ} 13^{\prime} \mathrm{N}, 16^{\circ} 12^{\prime} \mathrm{E}$; 18 Mar. 2010; D.J. Harris 9652; E • Bomassa, park headquarters; $2^{\circ} 12^{\prime} \mathrm{N}$, 16¹' E; 21 Mar. 2010; D.J. Harris 9664; E[E00397442] • Nouabalé-Ndoki National Park, 28 km NE of Bomassa; $2^{\circ} 21^{\prime} \mathrm{N}, 16^{\circ} 21^{\prime} \mathrm{E}$; 5 May 2018; D.J. Harris 10181; E・ Ouesso; $1^{\circ} 36^{\prime} \mathrm{N}, 1^{\circ} 03^{\prime} \mathrm{E}$; $11 \mathrm{Feb}$. 1970; Inventaire Ouesso 21; P • ca $12.5 \mathrm{~km} \mathrm{NE}$ of Kabo; $2^{\circ} 07^{\prime} \mathrm{N}, 16^{\circ} 10^{\prime} \mathrm{E}$; 13 Sep. 2006; G. Moukassa 182; E • ca $16 \mathrm{~km} \mathrm{NE}$ of Kabo; $2^{\circ} 07^{\prime} \mathrm{N}, 16^{\circ} 13^{\prime} \mathrm{E}$; 27 Sep. 2006; G. Moukassa 349; E • ca $2 \mathrm{~km} \mathrm{~S}$ of Kabo; $2^{\circ} 02^{\prime}$ N, $16^{\circ} 06^{\prime}$ E; 3 Oct. 2006; G. Moukassa 406; E・ ibid.; 4 Oct. 2006; G. Moukassa 421; E • ibid.; 4 Oct. 2006; G. Moukassa 422; E - ibid.; 4 Oct. 2006; G. Moukassa 424; E - ibid.; 5 Oct. 2006; G. Moukassa 465; E - ca 6.5 km SE of Kabo; $1^{\circ} 59^{\prime}$ N, $16^{\circ} 05^{\prime}$ E; 16 Oct. 2006; G. Moukassa

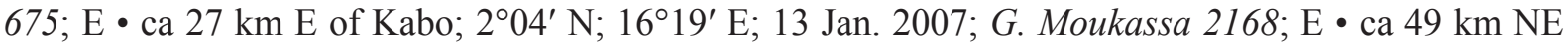
of Kabo, Nouabalé-Ndoki National Park; 2²2' N, 16²3' E; 26 Mar. 2007; G. Moukassa 3367; E • ibid.; 27 Mar. 2007; G. Moukassa 3422; E • ca 39.5 km NE of Kabo, Nouabalé-Ndoki National Park; $2^{\circ} 15^{\prime} \mathrm{N}, 16^{\circ} 22^{\prime} \mathrm{E}$; 14 Apr. 2007; G. Moukassa 3668; E • ca $24 \mathrm{~km}$ ENE of Kabo; $2^{\circ} 06^{\prime} \mathrm{N}, 1^{\circ} 17^{\prime} \mathrm{E}$; 24 May 2007; G. Moukassa 4506; E - village Bomassa; $2^{\circ} 12^{\prime}$ N, $16^{\circ} 12^{\prime}$ E; 5 Feb. 2013; S.T. Ndolo Ebika 1003; E[E00757774], WAG[WAG.1576295] • ca $12.5 \mathrm{~km} \mathrm{NE}$ of Kabo; $2^{\circ} 07^{\prime} \mathrm{N}, 16^{\circ} 10^{\prime} \mathrm{E}$; 8 Sep. 2006; F.O. Nzolani Silaho 166; E • ca $6.5 \mathrm{~km} \mathrm{SE}$ of Kabo; $1^{\circ} 59^{\prime} \mathrm{N}, 16^{\circ} 05^{\prime} \mathrm{E} ; 10$ Oct. 2006; F.O. Nzolani Silaho 510; E • ca 29 km E of Kabo; $2^{\circ} 04^{\prime}$ N, $16^{\circ} 20^{\prime}$ E; 8 Jan. 2007; F.O. Nzolani Silaho 1959; E • ibid.; 8 Jan. 2007; F.O. Nzolani Silaho 1969; E • ibid.; 9 Jan. 2007; F.O. Nzolani Silaho 2023; E • ca 5 km E of Kabo; $2^{\circ} 01^{\prime}$ N, $16^{\circ} 07^{\prime}$ E; 12 Feb. 2007; F.O. Nzolani Silaho 2594; E • ibid.; 14 Feb. 2007; F.O. Nzolani Silaho 2635; E • ca 5 km NE of Kabo, Nouabalé-Ndoki National Park; $2^{\circ} 17^{\prime}$ N, $16^{\circ} 23^{\prime}$ E; 19 Apr. 2007; F.O. Nzolani Silaho 3837; E • ca $14 \mathrm{~km}$ E of Kabo; $2^{\circ} 00^{\prime} \mathrm{N}, 16^{\circ} 12^{\prime} \mathrm{E}$; 3 May 2007; F.O. Nzolani Silaho 3862; E • ibid.; 10 May 2007; F.O. Nzolani Silaho 4115; E • ca $24 \mathrm{~km}$ ENE of Kabo; $2^{\circ} 06^{\prime} \mathrm{N}, 16^{\circ} 17^{\prime} \mathrm{E}$;

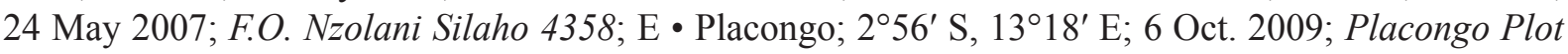
Series 102; K・ Mar. 1920; C.H.O. Pobéguin 81; P• Ouesso; $1^{\circ} 36^{\prime}$ N, $16^{\circ} 03^{\prime}$ E; Jul. 1963; F. Sandberg 58; P•Apr. 1891; Shelley 4018; P•Modzaka; $1^{\circ} 42^{\prime}$ N, $18^{\circ} 01^{\prime}$ E; 1889; F.-R. Thollon 24; P.

DEMOCRATIC REPUBLIC OF CONGO • Yangambi; $0^{\circ} 46^{\prime}$ N, 242ㄱ' E; 6 Feb. 1959; P. Bamps 332; BR[BR0000019964652] • Yailunga; 2²' S, 254' E; 17 Apr. 1959; P. Bamps 536; BR[BR0000019964669] - Yangambi; $0^{\circ} 46^{\prime} \mathrm{N}, 24^{\circ} 27^{\prime} \mathrm{E}$; 18 Aug. 1959; P. Bamps 674; BR[BR0000019964676] • downstream of Barumbu; $1^{\circ} 14^{\prime}$ N, $23^{\circ} 29^{\prime}$ E; 27 Oct. 1913; J.C. Bequaert 977; BR[BR0000019964683] 3 sheets • 1918; J.C.Bequaert 1646;BR[BR0000019964737]2 sheets •1919; J.C. Bequaert3167; BR[BR0000019964713] 2 sheets - Maniema; 1908; A. Berger 1909; BR[BR0000019964751] - Nala; 252' N, 27³9' E; 1911; A. Boone 39; BR[BR0000019964775] • Yaengo, Lobaye River; $0^{\circ} 28^{\prime} \mathrm{N}, 24^{\circ} 11^{\prime} \mathrm{E}$; 1 Jun. 2010; Boyekoli Ebale Congo 2010 Expedition 1157; BR[BR0000005145980] • La Kulu; 3²8' S, 2341' E; 1929; J.F. van den Brande 30; BR[BR0000020059606] • Plateau of Bateke road Kenge-Kinshasa; H. Breyne 380; BR[BR0000019964287] - road Maluku-Menkao km 9 Terr. Kin. Maluku; 40' S, 1540' E; H. Breyne 768; BR[BR0000019964294] • Yangami (plateau Isalowe); 046’ N, 242 27' E; 12 Feb. 1979; H. Breyne 3621; BR[BR0000019964782] - Kisantu Lemfu; 57' S, 15²' E; 14 Feb. 1952; H. Callens 2995; BR[BR0000019964300] - Lukulela, Congo River; $1^{\circ} 05^{\prime}$ S, $17^{\circ} 20^{\prime}$ E; 5 Jan. 1931; J.P. Chapin 648; BR[BR0000019964799] • Barumbu; 1921; J. Claessens 4 (new series); BR[BR0000019964805] • Katembo; 505' S, 26²2' E;1921; J. Claessens 68; BR[BR0000019964645] • Lowani; 15 Feb. 1910; J. Claessens 483; BR[BR0000019964829] 2 sheets - Buta; 2०47' N, 244' E; J. Claessens 677;

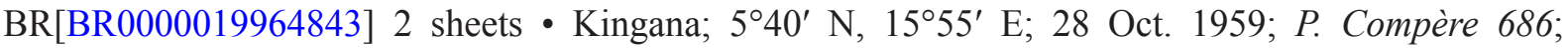
BR[BR0000019964317] - Eala; 004' N, 18¹8' E; 16 Mar. 1932; A. Corbisier-Baland 1236; BR[BR0000019964867] 2 sheets - Binga; 2²2' N, 20²9' E; 15 Nov. 1946; J. Croegaert 41; 
BR[BR0000019964881] • Bas Uele; 13 Nov. 1934; A. Dewulf 337; BR[BR0000019964942] 2 sheets • Botsima, Parc Salonga, river bank station; $1^{\circ} 09^{\prime}$ N, 21 ${ }^{\circ} 57^{\prime}$ E; 28 Feb. 1991; M. Dhetchuvi 803; BR[BR0000019964966] - Yangambi; 046' N, 24²7' E; 21 Sep. 1950; C.A. Donis 2776; BR[BR0000019964973], M • ibid.; 29 Sep. 1950; C.A. Donis 2836; BM, BR[BR0000019964980], P • ibid.; 29 Sep. 1950; C.A. Donis 2843; BR[BR0000019964997] • ibid.; 10 May 1951; C.A. Donis 3016; BR[BR0000019965000] • ibid.; 20 Dec. 1951; C.A. Donis 3222; BR[BR0000019965017] • ibid.; 20 Dec. 1951; C.A. Donis 3349; BR[BR0000019965024] - Bambesa; 326' N, 2541' E; 9 May 1944; H. du Bois 915; BR[BR0000019965048] • ibid.; 25 Jun. 1942; H. du Bois 261; BR[BR0000019965031] - Kingana; 540' S, 1555' E; 24 Jun. 1958; J. Dubois 317; BR[BR0000019964324], K • "Territoire de" [territory of] EkotaBokutuBoende; $3^{\circ} 26^{\prime} \mathrm{N}, 25^{\circ} 41^{\prime} \mathrm{E}$; 15Sep.1934;L.Dubois 610;BR[BR0000019965055] 2 sheets - Bodangabo; 374' N, 2050' E; 15 Feb. 1955; C. Evrard 217; BR[BR0000019965079] • "Vallée de la Loeka" [Loeka Valley]; C. Evrard 3448; BR[BR0000019965086] 2 sheets - Road Bomandja, Ikelambe source; 9 Jun. 1958; C. Evrard 4210; BR[BR0000019965109] • Nioki; 2०43' S, 17²4' E; Jul. 1941; A. Flamigni 6116; BR[BR0000019964386], K • Buna; 314' S, $18^{\circ} 59^{\prime}$ E; A. Flamigni

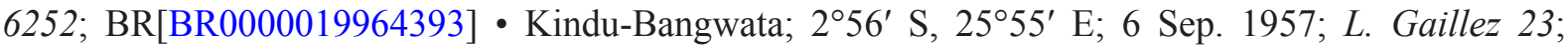
BR[BR0000019965116] - Elundu-Kindu km 37; 2 ${ }^{\circ} 56^{\prime}$ S, 25 $5^{\circ} 55^{\prime}$ E; 28 Sep. 1957; L. Gaillez 48; BR[BR0000019965123] •Bambesa;3²6'N,2541'E;1 Dec.1952;P.Gérard501;BR[BR0000019965130] - ibid.; 27 Mar. 1953; P. Gérard 566A; BR[BR0000019965147] • ibid.; 3 Oct. 1956; P. Gérard 2489; BR[BR0000019965154] • Prov. Orientale, Ango, Digba-Ango; 4²3' N, 2548' E; 5 Nov. 1963; P. Gérard 5592; BR[BR0000020059422], WAG[WAG.1845719] - Busiro River, Equateur; Jun. 1936; J.H.P.A. Ghesquière 2689; BR[BR0000019965178] 2 sheets, K • Yasouka; 15 Nov. 1938; G. Gilbert DIFOR1417; BR[BR0000019965291] 2 sheets • G. Gilbert 46; BR[BR0000019965208] • G. Gilbert 706; BR[BR0000019965222] 7 sheets - Route Bengamisa km 34; $0^{\circ} 56^{\prime} \mathrm{N}, 25^{\circ} 12^{\prime} \mathrm{E} ; 12$ May 1936; G. Gilbert 2303; BR[BR0000019965345] 2 sheets, K, P $\bullet$ Yangambi; $0^{\circ} 46^{\prime}$ N, $24^{\circ} 27^{\prime}$ E; 1948; G. Gilbert 8614; BR[BR0000019965369] 2 sheets • ibid.; 1948; G. Gilbert 8627; BR[BR0000019965383] • ibid.; 1948; G. Gilbert 8651; BR[BR0000019965390] • ibid.; 1949; G. Gilbert 8692; BR[BR0000019965406] • ibid.; 1948; G. Gilbert 8708; BR[BR0000019965413] • ibid.; 1948; G. Gilbert 8843; BR[BR0000019965420] • ibid.; 1948; G. Gilbert 8920; BR[BR0000019965437] • ibid.; 1948; G. Gilbert 8991; BR[BR0000019965444] • ibid.; 1948; G. Gilbert 9098; BR[BR0000019965451] • ibid.; 1948; G. Gilbert 9758; BR[BR0000019965468] • ibid.; G. Gilbert 10205; BR[BR0000006608378] • ibid.; G. Gilbert 10555; BR[BR0000006860172] • Bankaie; 2²2' S, $18^{\circ} 25^{\prime}$ E; 10 Jun. 1953; G. Gilbert 14058;

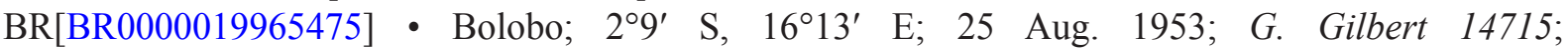
BR[BR0000019965482] • Beni; $0^{\circ} 29^{\prime}$ N, $2^{\circ} 28^{\prime}$ E; 2 Nov. 1931; J. Gillardin 52; BR[BR0000020059477] - Sangaie-Sankuru; 459' S, 23³0' E; 1934; J. Gillardin 134; BR[BR0000019964409] 2 sheets, K, P • Port Francofin; 4¹9' S, 20³5' E; May 1938; J. Gillardin 386; BR[BR0000019964423], K • Sangaie; $4^{\circ} 57^{\prime}$ S, 2333' E; J. Gillardin 537; BR[BR0000019964430] 2 sheets • Mukumari "Territoire de Lomela" [Lomela Territory]; May 1939; J. Gillardin 563; BR[BR0000019965499] 2 sheets • Madina Forest; P. Gille 272; BR[BR0000006608705] 2 sheets • J. Gillet 9; BR[BR0000019964348] • Kinshasa; 4¹8' S, $15^{\circ} 18^{\prime} \mathrm{E}$; 1902; J. Gillet 1625; BR[BR0000019964362] • between Kisantu and Kwango; 5 ${ }^{\circ}$ ' S, $15^{\circ} 4^{\prime}$ E; 1904; J. Gillet 3721; BR[BR0000019964379] • Likimi; 249’ N, 2044' E; Jan. 2013; S. de Giorgi 184;

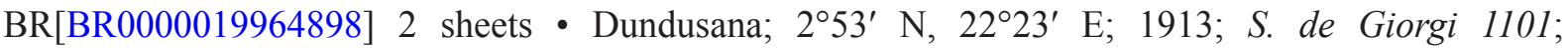
BR[BR0000019964904] 2 sheets - V. Goosens 2494; BR[BR0000019965512] - S. Gorbatoff 83; BR[BR0000019965529] • Mambasa;2²9' S, 26²7'E;6Apr. 1981; T.B. Hart30; BR[BR0000019965536], K・Kisantu; 57' S, 154' E; May 1905; F. van Houtte 2103 (in J. Gillet); BR[BR0000019964355] • Mbole; 26 Jun. 1944; R.P. Huelstaert 1311; BR[BR0000019965550] - Ebaa; 247' S, 18¹9' E; 1 Jun. 1951; E. Jans 933; BR[BR0000013319090] • "Bassin de 1'Epulu” [Epulu Bassin]; 1²3' N, 28³4' E; 17Jun. 1916; M.Lacomblez 7;BR[BR0000019965567]•1920;M.Lacomblezs.n.;BR[BR0000019965574] - Botoma Ikelemba; 055' N, 1958' E; 31 Jul. 1905; M. Laurent 1858; BR[BR0000019965581] • Eala; $0^{\circ} 3^{\prime}$ N, 18¹8' E; 15 Jun. 1906; M. Laurent 1866; BR[BR0000019965666] - Kasaï; 11 Nov. 1903; É. Laurent s.n.; BR[BR0000019964485] • 1 Jul. 1903; É. Laurent s.n.; BR[BR0000019964478] • close 
to Lié; 6 Jan. 1904; É. Laurent s.n.; BR[BR0000019964461] • Bolombo; 2 Dec. 1903; É. Laurent s.n.; BR[BR0000019965628] • Lulonga; 1904; É. Laurent s.n.; BR[BR0000019965635] • river bank below Basako; 1¹4' N, 2336' E; 10 Jan. 1903; É. Laurent s.n.; BR[BR0000019965604] - Kiri; 1²6' N, $19^{\circ} 0^{\prime}$ E; 6 Nov. 1903; É. Laurent s.n.; BR[BR0000019965611] • Bikoro; Jul. 1930; J.P.A. Lebrun 1453; BR[BR0000019965673] 2 sheets, U[U.1380070] • between Libenge and Congo, Ubangi; 3³9' N, $18^{\circ} 38^{\prime}$ E; Nov. 1930; J.P.A. Lebrun 1570; BR[BR0000020059446] • between Businga and Banzyville; $3^{\circ} 20^{\prime} \mathrm{N}, 20^{\circ} 53^{\prime} \mathrm{E}$; Jan. 1931; J.P.A. Lebrun 2008; BM, BR[BR0000019965697] • between Niangara and Wamba; $3^{\circ} 41^{\prime} \mathrm{N}, 27^{\circ} 52^{\prime}$ E; Jul. 1931; J.P.A. Lebrun 3262; BR[BR0000020059460] 2 sheets, P • Eala; $0^{\circ} 04^{\prime} \mathrm{N}, 18^{\circ} 18^{\prime} \mathrm{E}$; 1936; J. Leemans 150; BR[BR0000019965710] 2 sheets, K・Eala; $0^{\circ} 04^{\prime} \mathrm{N}, 18^{\circ} 18^{\prime} \mathrm{E}$; J.B. Lejeune s.n.; BR[BR0000019965734] - Kisangani, Île Kongolo, at junction of Lindi with River Zaïre; 27 Mar. 1977; J. Lejoly 1180; BR[BR0000019965741] • Likimi; 249’ N, 2044' E; 15 Sep. 1913; B. Lemaire 77; BR[BR0000019965758] • Yangambi; 046’ N, 24²7’ E; 22 Jul. 1958; A. Léonard 975; BR[BR0000019965765] - Walikale; 1 1 $25^{\prime} \mathrm{S}, 2^{\circ} 04^{\prime} \mathrm{E}$; 18 Jun. 1959; A. Léonard 4650; $\mathrm{BR}[\mathrm{BR} 0000019965772$ ], $\mathrm{K} \cdot$ Kampene; 335' S, 2640' E; 7 Aug. 1959; A. Léonard 5616; BR[BR0000019965789], K • Ikelamba; 1 Sep. 1946; J. Léonard 535; BR[BR0000019965796] • C. Léontovitch 121; BR[BR0000019965802] 2 sheets - Gemena, Mombolo; $3^{\circ} 15^{\prime} \mathrm{N}, 19^{\circ} 46^{\prime} \mathrm{E}$; 16 Oct. 1942; C. Léontovitch 226; BR[BR0000019965826], K・ Madibi; 4¹7’ N, 18²5’ E; 29 Jun. 1904; E. Lescrauwaet 114; BR[BR0000019964492] - Tumba; 28 Feb. 1957; L. Liben 2613; BR[BR0000019964508] - near Mwene-Ditu; 70' S, 2326' E; 4 Nov. 1957; L. Liben 3932; BR[BR0000019964515] - Budjalibala; 25 Dec. 1926; D.H. Linder 1793; K, P • Yangambi; 046' N, $24^{\circ} 27^{\prime} \mathrm{E}$; 1935; J. Louis 320; BM, BR[BR0000013255787] 2 sheets, K・Yangambi, km 6 road to Ngazi; $0^{\circ} 46^{\prime} \mathrm{N}, 24^{\circ} 27^{\prime}$ E; 26 Oct. 1935; J. Louis 434; BR[BR0000013255770] 2 sheets $\bullet$ Yangambi, km 8 on the road to Ndaze; $0^{\circ} 46^{\prime} \mathrm{N}, 24^{\circ} 27^{\prime} \mathrm{E}$; 7 Dec. 1935; J. Louis 778; BR[BR0000013255763] 2 sheets, K, P - "Plateau de la Lusambila" [Lusambila Plateau]; 5²3' S, 27¹8' E; 24 Mar. 1936; J. Louis 1529; BR[BR0000013255756] 2 sheets, K, P • Yangambi, $5 \mathrm{~km} \mathrm{~N}$ of river; $0^{\circ} 46^{\prime} \mathrm{N}, 2^{\circ} 27^{\prime} \mathrm{E} ; 20$ May 1937; J. Louis 3922; BR[BR0000020059002] - Yangambi, at Tofende; $0^{\circ} 46^{\prime} \mathrm{N}, 2^{\circ} 27^{\prime} \mathrm{E} ; 25$ Aug. 1939; J. Louis 15918; BR[BR0000020059026] 3 sheets - Sankuru Forest; 15 Jun. 1903; P.E. Luja 15; BR[BR0000019964522] 2 sheets $\bullet$ Ndumba River; $1^{\circ} 10^{\prime} \mathrm{N}, 2^{\circ}{ }^{\circ} 14^{\prime} \mathrm{E}$; 3 Nov. 2004; W.R.Q. Luke 10514z;

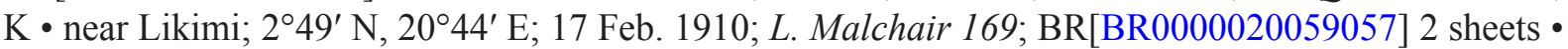
Likimi Forest; $2^{\circ} 49^{\prime} \mathrm{N}, 20^{\circ} 44^{\prime} \mathrm{E}$; 1910; L. Malchair 422; BR[BR0000020059071] • Yangambi; $0^{\circ} 46^{\prime} \mathrm{N}$, 24²7’ E; 8 Sep. 1952; E. Maudoux 378; BR[BR0000020059095] • ibid.; 9 Sep. 1952; E. Maudoux 385; BR[BR0000020059088] -Kikwit; 502'S, 1848'E; 8Aug. 1991; B. Masens 756; BR[BR0000019964546], WAG[WAG.1845711] • Dundusana; 2 ${ }^{\circ} 53^{\prime} \mathrm{N}, 2^{\circ} 23^{\prime} \mathrm{E} ; 1913 ;$ M. G. Mortehan 71; BR[BR0000020059125] - near Bolanda Village; 20 Oct. 1913; A. Nannan 5; BR[BR0000020059132] • Wamba; 16 Nov. 1988; Nsola 1140; BR[BR0000020061043] • between Mwenda and Kalasabango; 0²4' N, 2946' E; 8 Sep. 1952; H.A. Osmaston 2490A; BM, BR[BR0000020059484] • km 215 road Kavumu-Walikale; $1^{\circ} 27^{\prime} \mathrm{N}$, $28^{\circ} 50^{\prime}$ E; 19 Aug. 1955; R. Pierlot 937; BR[BR0000020059163] • near Bambesa Uele; $3^{\circ} 26^{\prime}$ N, $25^{\circ} 41^{\prime}$ E; 1936; R. Pittery 783; BR[BR0000020059170] • Eala; $0^{\circ} 03^{\prime}$ N, $18^{\circ} 18^{\prime}$ E; 11 Nov. 1906; L. Pynaert 643; BR[BR0000020059194] • ibid.; 15 May 1907; L. Pynaert 1279; BR[BR0000020059217] • ibid.; 15 Sep. 1907; L. Pynaert 1709; BR[BR0000020059231] - Mobwasa; $2^{\circ} 35^{\prime}$ N, $23^{\circ} 08^{\prime}$ E; 15 Oct. 1913 ; F. Reygaert 1054; BR[BR0000020059255] • ibid.; 15 Oct. 1913; F. Reygaert 1088; BR[BR0000020059248] • Liwea; 448' N, 1904' E; 16 Nov. 1925; W. Robyns 994; BR[BR0000020059262] 2 sheets • River Rubi Station; 9 Dec. 1929; W. Robyns 1136; BR[BR0000020059286] 3 sheets • Lapku; 1906; A. Sapin s.n.; BR[BR0000019964560] • Madibi; 1 Jul. 1906; A. Sapin s.n.; BR[BR0000019964553] • Madilu; 1907; A. Sapin s.n.; BR[BR0000019964577] • Bwado; 1912; A. Sapin s.n.; BR[BR0000020059347] • between Lubwe and Bemna-Makina; 1910; A. Sapin s.n.; BR[BR0000019964584] - Eala; 04' N, 18¹8' E; 15 Nov. 1930; P. Staner 1497; BR[BR0000020059354] 2 sheets • Gombe; 22 Apr. 1959; L. Toka 49; BR[BR0000020059385] - Lukolela/Mompoto; $1^{\circ} 05^{\prime}$ S, 17²0' E; 13 Jul. 1959; L. Toka 132; BR[BR0000020059378] - Kikwit; 502' S, 18 49' E; 15 Jan. 1914; H. Vanderyst 2872; BR[BR0000019964614] • ibid.; 15 Jan. 1914; H. Vanderyst 3121; BR[BR0000019964621] • Kisantu 
Wombali; 15 Nov. 1910; H. Vanderyst s.n.; BR[BR0000019964607] • Ipamu; 1922; H. Vanderyst s.n.; BR[BR0000019964638] - Mobwasa; 2³9' N, 2303' E; 10 May 1914; F.M.C. Vermoesen 372; BR[BR0000020059613] 3 sheets, P • Bambesa Bokamba; $3^{\circ} 26^{\prime} \mathrm{N}, 25^{\circ} 41^{\prime} \mathrm{E}$; 16 Apr. 1940; J.M. Vrydagh 337;BR[BR0000020059637]•Bokondji,Lometa;20May 1959;P.deWanckel65;BR[BR0000019964928] - Parc National Albert Kigoma, near Balozi; 6³3' S, 28²2' E; 15 Jul. 1954; G.F. de Witte 10796; BR[BR0000019964935] - Yangambi; $0^{\circ} 46^{\prime} \mathrm{N}, 2^{\circ} 27^{\prime}$ E; 9 Dec. 1960; F. Yafunga 23; BR[BR0000020059408], K.

EQUATORIAL GUINEA • near Bocoricho Balachá; 3²5' N, 8³4' E; 13 Aug. 1986; M.F. de Carvalho 2290; U[U.1380067], WAG[WAG.1845829].

GABON • "Env. de Libreville" [surroundings of Libreville]; Jun. 1913; H. Courtet s.n.; L[L.2349655]

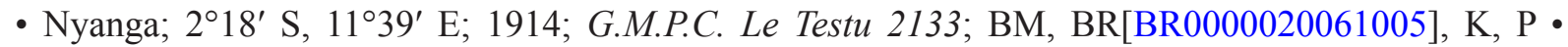
Lastoursville; $0^{\circ} 49^{\prime} \mathrm{S}, 12^{\circ} 46^{\prime} \mathrm{E}$; 28 May 1929; G.M.P.C. Le Testu 7106; BM, BR[BR0000020061012], P 5 Dec. 1929; G.M.P.C. Le Testu 7738; BM, P - 7 May 1930; G.M.P.C. Le Testu 8077; BR[BR0000019964256], P • "Reserve des Monts Doudou, Sud-Ouest de la Brigade de Moukalaba" [Monts Doudou Reserve, SW of Brigade of Moukalaba], village Mourindi; 2 ${ }^{\circ} 34^{\prime}$ S, 10 $10^{\circ} 44^{\prime}$ E; 22 Mar. 2000; M.S.M. Sosef 935; WAG[WAG.1834958] - Nyanga, Mourindi; $2^{\circ} 19^{\prime}$ S, $10^{\circ} 26^{\prime}$ E; 18 Apr. 2000; M.S.M. Sosef 1338; P • F.-.R. Thollon 152; BR[BR0000020060985], K, P.

GHANA • near dam on WACRI station; $6^{\circ} 44^{\prime} \mathrm{N}, 1^{\circ} 36^{\prime} \mathrm{W} ; 2$ Nov. 1953; Brewu WACRI6831; K, P • Akim Swedru, Awisa; 553' N, $1^{\circ} 00^{\prime}$ W; Dec. 1933; F.R. Irvine 2079; E[E00930369] • BIA National Park and Production Reserve, at Kumkumso Park Guard Camp; 6³7' N, 304' W; 28 Feb. 1996; H.H. Schmidt 2013; WAG[WAG.1845666].

GUINEA • N'Zérékoré; 745' N, 849' W; Apr. 1944; J.G. Adam 69; P • Nimba Mountains, Gba Valley; $7^{\circ} 41^{\prime} \mathrm{N}, 8^{\circ} 24^{\prime} \mathrm{W} ; 28$ Jun. 2007; C.C.H. Jongkind 7876; WAG[WAG.1845696] • Bossou; 7³8' N, $8^{\circ} 30^{\prime}$ W; Jul. 1942; R. Schnell 1406; P • Iasso-Offin; Jan. 1928; C. Vigne 1011; P.

IVORY COAST • Man; 7²4' N, 7³3' W; A. Aubréville 959; BR[BR0000019964102], K, P • Pinhou; $6^{\circ} 37^{\prime} \mathrm{N}, 7^{\circ} 20^{\prime} \mathrm{W}$; Jan. 1969; P. Bamps 1964; BR[BR0000019964096], K • Bouroukrou; 740' N, $3^{\circ} 12^{\prime}$ W; 5 Jan. 1907; A.J.B. Chevalier 16844; K, P - Longan; 26 Mar. 1907; A.J.B. Chevalier 17685; P - Moyen-Comoé, between Ebrinakoué and Diangobo; $6^{\circ} 34^{\prime}$ N, $3^{\circ} 20^{\prime}$ W; 20 Dec. 1909; A.J.B. Chevalier 22618; P.

LIBERIA • 13 Dec. 1945; W.J. Harley s.n.; WAG[WAG.1845669] - Zuole area, near Tapeta; $6^{\circ} 41^{\prime}$ N, $8^{\circ} 47^{\prime}$ W; 28 Jan. 1962; A.G. Voorhoeve 769; WAG[WAG.1845667].

NIGERIA • Hunata Ibene; 23 Feb. 1966; J.O. Ariwaodo 1123; K・ Okumu Forest Reserve; $6^{\circ} 15^{\prime}$ N, $5^{\circ} 06^{\prime}$ E; 19 Dec. 1947; J.P.M. Brenan 8542; K - Okomu Forest Reserve; 6¹5' N, 506 ${ }^{\circ}$ E; 27 Feb. 1948; J.P.M. Brenan 9174; BM, K, P • Ikom; 557' N, 842' E; 1935; R. Catterall 71; K・ Moor Plantation-Ibadan; 4 Apr. 1966; C.L.M. van Eijnatten 1326; WAG[WAG.1845726] 3 sheets - Ibadan N.A. Forest Reserve, Mamu; 28 Apr. 1948; Idahosa FHI22853; K • Akure Forest Reserve, Aponmu high forest; $7^{\circ} 14^{\prime} \mathrm{N}, 5^{\circ} 03^{\prime} \mathrm{E} ; 28$ Oct. 1949; R.W.J. Keay FHI25494; K - Akure Forest Reserve, Aponmu; 1 Nov. 1949; R.W.J. Keay FHI25532; K・ Sapoba; 606’ N, 553' E; J.D. Kennedy 1649; BM, BR[BR0000019964126], K・J.D. Kennedy 1861; BM, BR[BR0000019964133] 2 sheets • Mamu Forest Reserve; $7^{\circ} 05^{\prime} \mathrm{N}, 3^{\circ} 55^{\prime} \mathrm{E}$; 6 Sep. 1944; C.F.A. Onochie FHI7661; K・ Ggoja District; D.R. Rosevear 58/29; K・Sapoba Reserve; $6^{\circ} 06^{\prime}$ N, 553' E; 28 Nov. 1934; A.F. Ross 233; K・ Nikrowa, Okomu Reserve; 6¹4' N, 5²1' E; 28 Jan. 1935; A.F. Ross 249; K・ Sapoba, Benin; 606' N, 553' E; 29 Mar. 1935; R. Ross 173; BM・H.G. Stubbings 148; K・Degama; 1914; P.A. Talbot 3637; BM. 


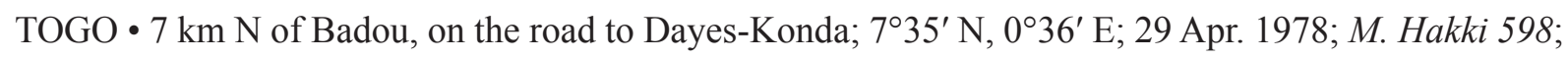
$\mathrm{K}, \mathrm{P}$.

UGANDA • Bundibugyo; $0^{\circ} 44^{\prime}$ N, 30²' E; 23 Nov. 1991; R. Dechamps 11635; BR[BR0000019964270] - Budongo Forest; $1^{\circ} 38^{\prime}$ N, $31^{\circ} 35^{\prime}$ E; Feb. 1935; W.J. Eggeling 1506; BR[BR0000019964263] • Bwamba Forest; $0^{\circ} 50^{\prime}$ N, 30³' E; Feb. 1935; W.J. Eggeling 1607; K - Bwamba Forest; 050' N, 30³' E; 1 Feb. 1945; P.J. Greenway 7063; K.

\section{Description}

Haвiт. Tree, to 4-12 m tall, DBH to (5-)10-40 cm; many-branched, branches and twigs slightly tomentose or glabrous.

LEAVES. With 2-3-fid stipules, divided for part of their length into mostly ovate-lanceolate lobes, 5-12 mm long, 2-3 mm wide at base, sharply pointed, glabrous or slightly tomentose; petiole $0.5-1.8 \mathrm{~cm}$ long, almost glabrous with rusty indumentum; leaf blade usually drying greenish-brown, oblong-oblanceolate to oblong-elliptic, $10-35 \mathrm{~cm}$ long, 3.5-14 cm wide, sub-leathery, glabrous above, mostly glabrous below apart from some scattered single hairs on midrib or some scattered stellate hairs on blade, apex acute to slightly acuminate, base cuneate to rounded, often asymmetric, joining the petiole at different points on the two sides of the lamina, margin coarsely and irregularly toothed with large (up to $1 \mathrm{~cm}$ ), jagged teeth, with domatia formed of long simple hairs in nerve axils below (but can be lacking).

INFLORESCENCE. An axillary or terminal cyme of 5-7 flowers, usually rather condensed; peduncle $0.7-$ $2 \mathrm{~cm}$ long, glabrous; involucral bracts 5-6, ovate-lanceolate, 3-5 mm long, greyish tomentose, not enveloping the young buds, usually falling off early.

FLowers. Pedicellate; pedicels thick, 2-6 mm long, slightly tomentose; buds subglobose, greyishtomentose; sepals white or cream to slightly pale pink, fleshy, oblong-lanceolate, $1-1.4 \mathrm{~cm}$ long, $4-6 \mathrm{~mm}$ wide, fringed at apex, tomentose; petals oblong-roundish, 2.4-2.8(-4) mm long, 2-2.4 mm wide; ovary 6-10-locular.

Fruits. Subglobose to elliptic, $10-25 \mathrm{~cm}$ long, $8-20 \mathrm{~cm}$ wide, flattened at base and apex, obscurely grooved, glabrous.

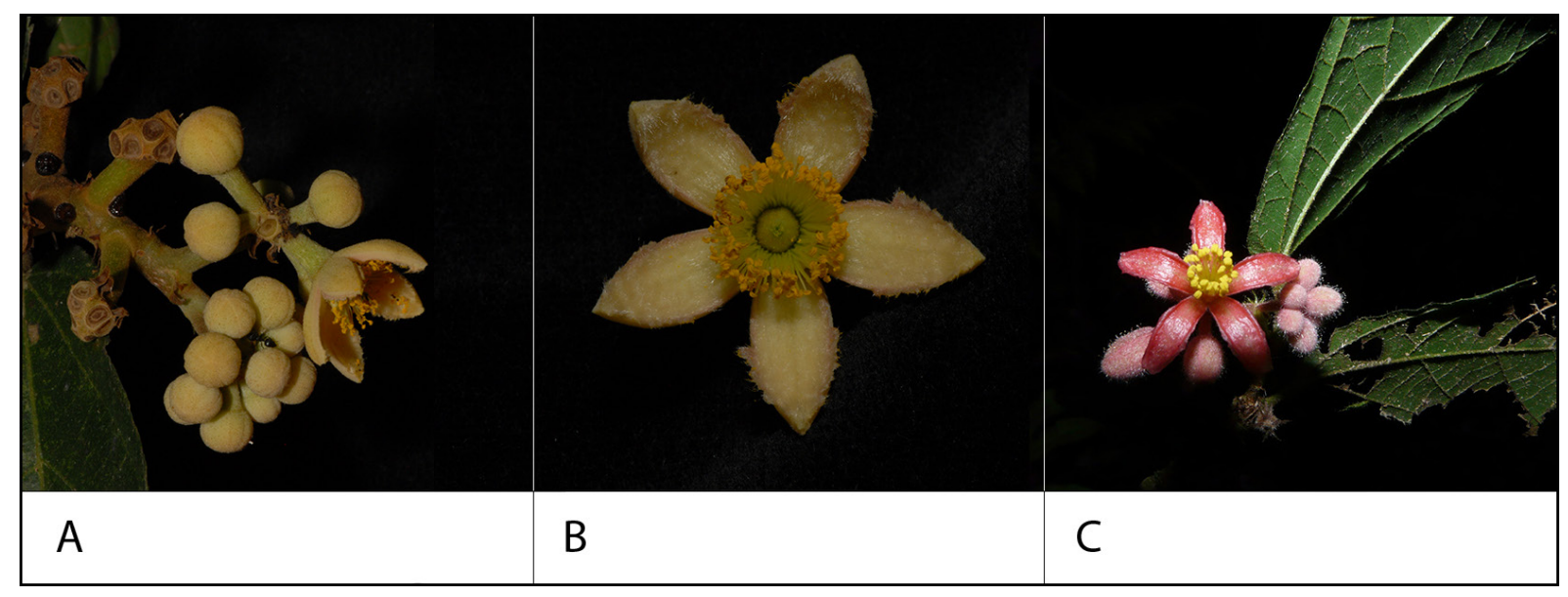

Fig. 6. Desplatsia Bocq. flower images. A-B. D. dewevrei (De Wild. \& T.Durand) Burret (Harris 9664 (E)), images by D. Harris (RBGE). C. D. subericarpa Bocq., image by Gilles Dauby. 
SEeds. Obovoid, $1.8 \mathrm{~m}$ long, $8 \mathrm{~mm}$ wide.

Distribution (see Fig. 7)

Angola, Cameroon, Central African Republic, Congo, Democratic Republic of Congo, Equatorial Guinea, Gabon, Ghana, Guinea, Ivory Coast, Liberia, Nigeria, Togo, Uganda.

\section{Habitat}

Terra firma forest and occasionally recorded from seasonally flooded forest. At altitudes of 350-1250 m a.s.1.

\section{Phenology}

Flower: February-April, September, fruit: February, July, October-November.

\section{Proposed IUCN status}

Least Concern (LC). Desplatsia dewevrei has an EOO of $4271669 \mathrm{~km}^{2}$ and an AOO of $572 \mathrm{~km}^{2}$, with a wide distribution across the forests of West and Central Africa. There are no major threats. Several collections are from protected areas which give this species some level of protection. Our assessment as LC is in agreement with the IUCN Red List assessments of threatened species (BGCI and IUCN SSC Global Tree Specialist Group 2019a).

\section{Notes}

Two sheets of Dewèvre 901 collection are available at BR, here we selected the better conserved sheet as lectotype for Grewiopsis dewevrei.

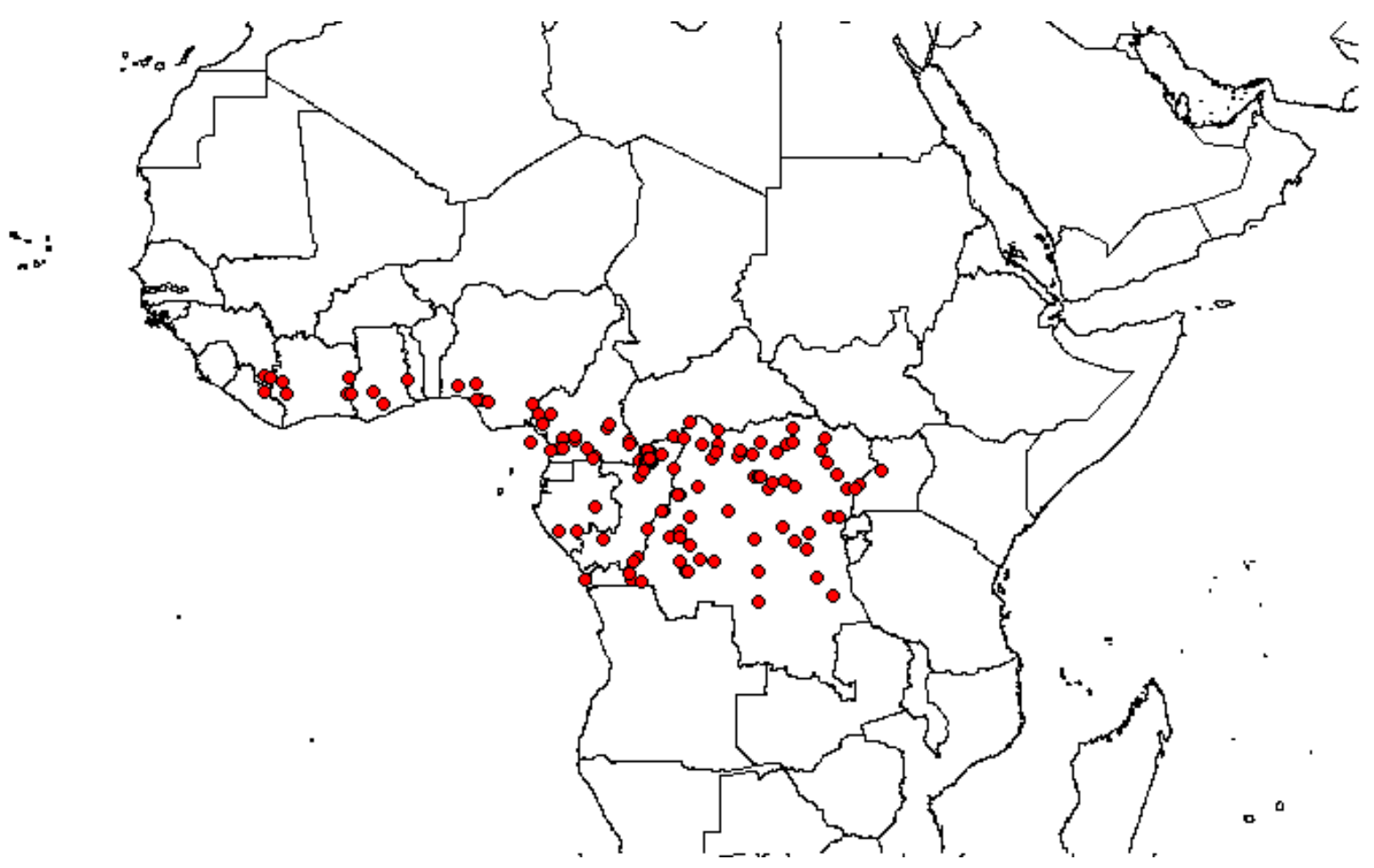

Fig. 7. Distribution map for Desplatsia dewevrei (De Wild. \& T.Durand) Burret. 
The sheet with fruits and flowers out of the two sheets found in Paris of the Trilles 189 collection cited in the protologue for Grewiopsis trillesiana syn. nov. (Chevalier 1912) was chosen here as lectotype for that name.

Out of the syntypes available at K, P and WAG, a specimen with flowers we have seen at $\mathrm{K}$ was chosen as lectotype for D. lutea.

Out of the two specimens available from the Chevalier 16272 collection for D. acuminata, the fruiting specimen was chosen as lectotype.

Desplatsia mildbraedii Burret

Figs 1D, 2F, 3D, 4E, 8, Table 1

Wissenschaftliche Ergebnisse der Deutschen Zentral-Afrika-Expedition, 1907-1908, unter Führung Adolf Friedrichs, Herzogs zu Mecklenburg. Vol. 2: Botanik: 497 (Mildbraed 1912). - Type: DEMOCRATIC REPUBLIC OF CONGO - Irumu, Kifuku; G.W.J. Mildbraed 2882; syntype: B, presumed $\uparrow$ - Turuma, Poyo; $2^{\circ} 42^{\prime}$ N, 30²1' E; Apr. 1908; G.W.J. Mildbraed 2836; syntype: B, presumed $\dagger$, lectotype: BM, designated here, isolectotype: BR[BR0000013205478].

\section{Other material studied}

CAMEROON • village of Mayouka, near Muguka; Jul. 1917; F. Fleury 33163; BR[BR0000020059491],P• $15 \mathrm{kmE}$ ofDimako; 4²3' N, 13³4' E; 15 Dec. 1965; A.J.M. Leeuwenberg 7348; BR[BR0000019964225],

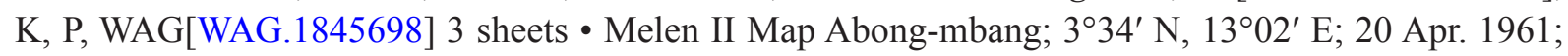
R. Letouzey 3877; BR[BR0000020438371], K, P, WAG[WAG.1845673] • near Ngola, $30 \mathrm{~km} \mathrm{E}$ of Yokadouma; 329' N, 15²19' E; 10 May 1963; R. Letouzey 4979; BR[BR0000019964201], K, P,

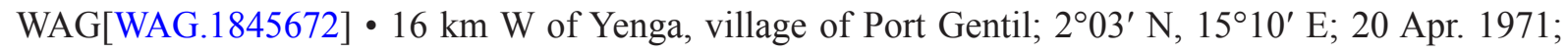
R. Letouzey 10697; BR[BR0000020059514], K, P.

CENTRAL AFRICAN REPUBLIC - Moussaboula Village, $7 \mathrm{~km} \mathrm{~N}$ of Bayanga; $3^{\circ} 55^{\prime} \mathrm{N}, 1^{\circ} 23^{\prime} \mathrm{E}$; 1 May 1985; R.W. Carroll 25; BR[BR0000020061104] • R.W. Carroll 1072; MO • R.W. Carroll 1084; MO • R.W. Carroll 1092; MO • Kongana camp; $2^{\circ} 47^{\prime}$ N, $16^{\circ} 25^{\prime}$ E; 16 Dec. 1993; D.J. Harris 4005; E[E00930972] • Kongana research camp; $2^{\circ} 47^{\prime}$ N, $16^{\circ} 16^{\prime}$ E; 1 Feb. 1994; D.J. Harris 4397; E[E00930950] - Sangha-Mbaere, Kongana camp; 247’ N, 16²6' E; 30 Jan. 1996; D.J. Harris 5408; E[E00930974] • Eleme ya Ngombe plot, W of Sangha River; $2^{\circ} 58^{\prime} \mathrm{N}, 16^{\circ} 11^{\prime} \mathrm{E} ; 3$ Dec. 2000; D.J. Harris 7303; E[E00930971] • W of Sangha River, $12 \mathrm{~km}$ NE of Bayanga; 301' N, 16¹1' E; 13 May 2001; D.J. Harris 7709; E[E00930973] • 30 Dec. 1948; Tisserant (Équipe) 1303; BM, BR[BR0000020059538], P• Boukoko; 354' N, 1755' E; 11 Jan. 1949; Tisserant (Équipe) 1324; BM, BR[BR0000020059521], P - Bayanga on the road to Dzanga; 2 ${ }^{\circ} 4^{\prime} \mathrm{N}, 16^{\circ} 1^{\prime}$ E; 20 Feb. 1976; Wraber LJU49486; K.

DEMOCRATIC REPUBLIC OF CONGO • Bambesa; 326’ N, 2541' E; 27 Mar. 1953; P. Gérard 566B; BR[BR0000020059552] • ibid.; 26 Feb. 1957; P. Gérard 2669; BR[BR0000020059545] 2 sheets - Epulu, "Zone de Mambesa (Ituri)" [area of Mambesa (Ituri)]; 1²5' N, 2835' E; 12 Mar. 1986; T. Hart 540; BR[BR0000019965543] • Nala; 252' N, 27³9’ E; 1907; F. Seret 789; BR[BR0000020059644] 4 sheets.

UGANDA 4 miles E of Mukono; $0^{\circ} 21^{\prime}$ N, 32 $2^{\circ} 48^{\prime}$ E; 20 Dec. 1951; H.C. Dawkins 685; K・ Budongo Forest; $1^{\circ} 38^{\prime} \mathrm{N}, 31^{\circ} 35^{\prime} \mathrm{E}$; W.J. Eggeling 1464 ; K • Budongo Forest, Bunyoro; $1^{\circ} 38^{\prime} \mathrm{N}$, $31^{\circ} 35^{\prime} \mathrm{E}$; $15 \mathrm{Jul}$. 1935; W.J. Eggeling 1745; BR[BR0000020059507] • ibid.; Jul. 1935; W.J. Eggeling 2131; K. 


\section{Description}

HaBiт. Tree to 9-25 m tall, DBH to (15-)40-80 cm, branches and twigs slightly tomentose and with scattered simple hairs.

Leaves. With 3-4-fid stipules, finely divided almost to the base, 5-10 $\mathrm{mm}$ long, $2 \mathrm{~mm}$ wide, lanceolate to filamentous, slightly rusty-tomentose and with scattered long hairs; petiole $1.2-1.8 \mathrm{~cm}$ long with similar indumentum; leaf blade usually drying greenish-brown, oblong, sub-leathery, 10-21 cm long, $2.8-8 \mathrm{~cm}$ wide, apex acute to slightly acuminate, base rounded to slightly asymmetrically subcordate, regularly toothed margin, slightly puberulous or almost glabrous above, densely, evenly-spaced stellate hairs below, with distinctly longer hairs on midrib and secondary veins, secondary veins rarely looping; domatia absent.

INFLORESCENCE. An axillary or terminal cyme of 4-8 pedicellate flowers, loose; peduncle $1-2.5(-4) \mathrm{cm}$ long covered by the same indumentum as the stipules; involucral bracts 6-8, 3 inner ovate-elliptic, $3 \mathrm{~mm}$ long, $2 \mathrm{~mm}$ wide, and 3-5 outer oblong-lanceolate, not enveloping in the buds and falling off early.

FLowers. Pedicellate, pedicels $0.8-2 \mathrm{~cm}$ long with similar indumentum as the stipules; buds elliptic and brown-velvety. Sepals cream to pale yellow, fleshy but brittle, $1-1.5 \mathrm{~cm}$ long, $2-4 \mathrm{~mm}$ wide, not fringed at tip, tomentose and with some longer hairs outside. Petals lanceolate to obovate-oblong, 4-4.8 mm long, 1-2.4(-3) mm wide. Ovary 5-7-locular.

FRUITs. Globose to subglobose, $6.5-9.5 \mathrm{~cm}$ long, 5-8.5 cm wide, obscurely grooved, glabrous.

SeEds. Obovoid, $0.7-1.7 \mathrm{~cm}$ long, $0.3-1 \mathrm{~cm}$ wide.

Distribution (see Fig. 8)

Cameroon, Central African Republic, Democratic Republic of Congo, Uganda.

\section{Habitat}

Terra firma forest, both old growth and selectively logged semi-evergreen forest. At altitudes of 3501200 m a.s.1.

\section{Phenology}

Flower: December-May, fruit: December, February, July.

\section{Proposed IUCN status}

Least Concern (LC). Desplatsia mildbraedii has an EOO of $441153 \mathrm{~km}^{2}$ and a wide distribution range across the north of the Congo basin. This species has a relatively small AOO of $80 \mathrm{~km}^{2}$, but since there are no major threats and the species has fairly recently been collected in protected areas of the DzangaSangha Reserve (Harris 2002; Harris \& Wortley 2008) and the Okapi Wildlife Reserve (Democratic Republic of Congo), thus ensuring some level of protection, D. mildbraedii is classed as Least Concern.

\section{Notes}

First-step lectotypification for D. mildbraedii was done by Wilczek (1963). Syntypes are available at BM and BR; here, we select the BM specimen as lectotype, as it bears flowers and fruits which we have examined in London.

Desplatsia mildbraedii is clearly recognized as a separate species by us despite doubts on the validity of earlier separations of D. mildbraedii from D. dewevrei (Verdcourt 2001). The key characters found during this revision that reliably set $D$. mildbraedii apart from $D$. dewevrei are shown in Table 1 . 
Table 1. Characters separating Desplatsia mildbraedii Burret from D. dewevrei (De Wild. \& T.Durand) Burret.

\begin{tabular}{|c|c|c|}
\hline & D. mildbraedii & D. dewevrei \\
\hline Tree height & up to $25 \mathrm{~m}$ & up to $12 \mathrm{~m}$ \\
\hline DBH & up to $80 \mathrm{~cm}$ & up to $40 \mathrm{~cm}$ \\
\hline Leaves & $\begin{array}{l}\text { obscurely toothed with teeth } \\
\text { generally }<1 \mathrm{~cm} \text {, blade with densely, } \\
\text { evenly-spaced stellate hairs below }\end{array}$ & $\begin{array}{l}\text { coarsely and irregularly toothed with large } \\
\text { (up to } 1 \mathrm{~cm} \text { ), jagged teeth, blade glabrous } \\
\text { to almost glabrous below (apart from some } \\
\text { scattered single hairs on the midrib and } \\
\text { some scattered stellate hairs on the blade) }\end{array}$ \\
\hline Domatia & absent & $\begin{array}{l}\text { leave blade often with domatia formed of } \\
\text { long simple hairs in nerve axils below }\end{array}$ \\
\hline Petioles & with scattered long hairs & no scattered long hairs \\
\hline Pedicels & $0.8-2 \mathrm{~cm}$ long & thick, 2-6 mm long \\
\hline Buds & elliptic, brown-velvety & subglobose, greyish-tomentose \\
\hline Petals & $\begin{array}{l}\text { lanceolate to obovate-oblong, } \\
4-4.8 \mathrm{~mm} \text { long, } 1-2.4(-3) \mathrm{mm} \text { wide }\end{array}$ & $\begin{array}{l}\text { oblong-roundish, } 2.4-2.8(-4) \mathrm{mm} \text { long, } \\
2-2.4 \mathrm{~mm} \text { wide }\end{array}$ \\
\hline Fruits & $6.5-9.5 \mathrm{~cm}$ long, $5-8.5 \mathrm{~cm}$ wide & $10-25 \mathrm{~cm}$ long, $8-20 \mathrm{~cm}$ wide \\
\hline
\end{tabular}

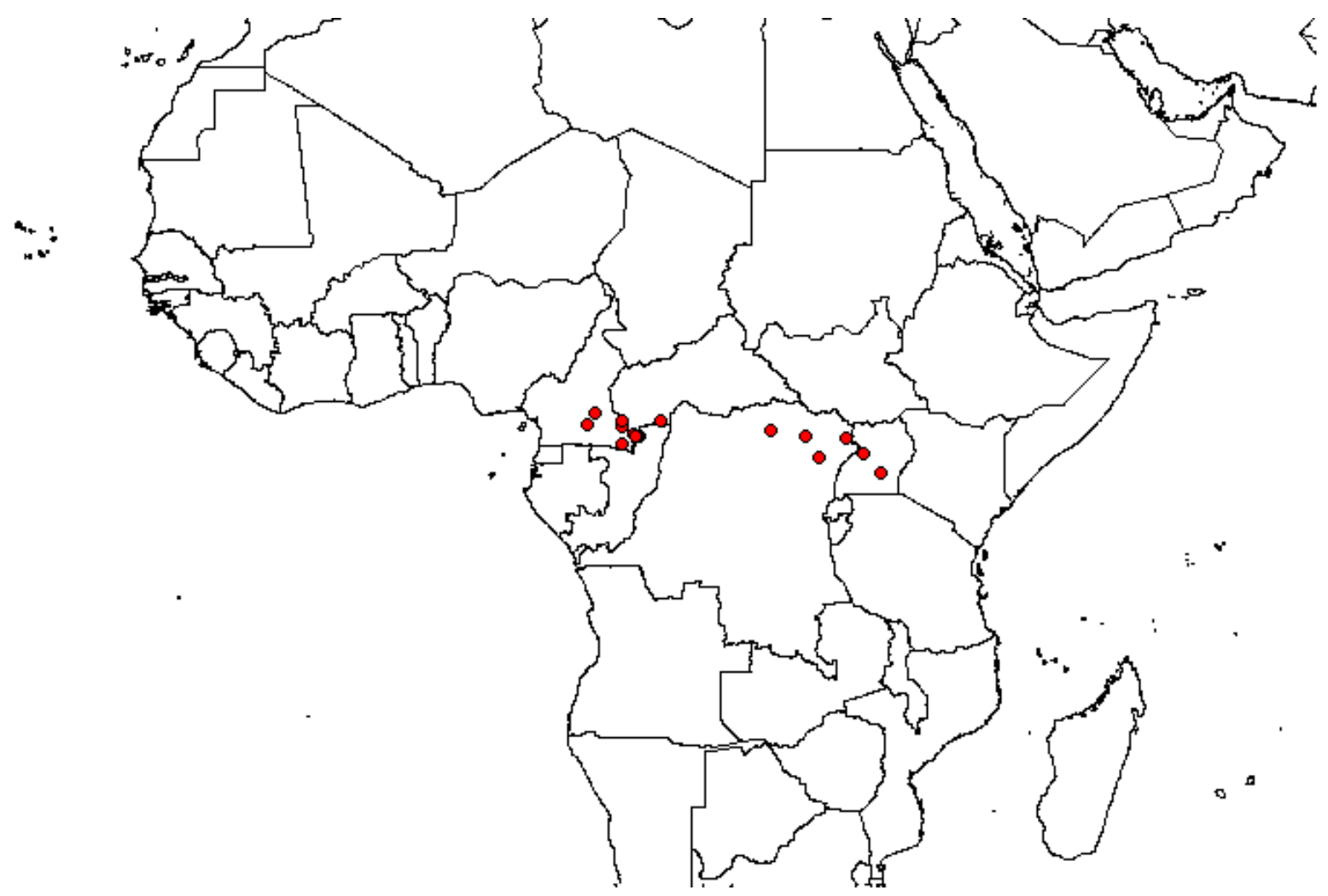

Fig. 8. Distribution map for Desplatsia mildbraedii Burret. 


\section{Desplatsia subericarpa Bocq.}

Figs 1A, 2C-D, 3A, 4F, 6C, 9

Adansonia 7: 51 (Bocquillon 1866). - Type: EQUATORIAL GUINEA - Jul. 1862; G. Mann 1695; lectotype: K[K000241997], designated here, isolectotypes: K[K000241998], K[K000241999], $\mathrm{P}[\mathrm{MNHN}-\mathrm{P}-\mathrm{P} 06724376], \mathrm{P}[\mathrm{MNHN}-\mathrm{P}-\mathrm{P} 06724377]$.

Desplatsia klainii Pierre ex A.Chev., Les végétaux utiles de l'Afrique tropicale française. Etudes scientifiques et agronomiques. Vol. 9: 81 (Chevalier 1917). - Type: GABON - 14 Aug. 1901; T.-J. Klaine 2290; lectotype: P[MNHN-P-P06724387], isolectotypes: K, P[MNHN-P-P06724388] • Libreville; $0^{\circ} 23^{\prime} \mathrm{N}, 9^{\circ} 27^{\prime} \mathrm{E}$; 8 Jan. 1902; T.-J. Klaine 2651; syntypes: BM, BR[BR0000020060015], $\mathrm{P}[\mathrm{MNHN}-\mathrm{P}-\mathrm{P} 06724381] 6$ sheets.

Grewiopsis globosa De Wild. \& T.Durand, Bulletin de la Société royale de Botanique de Belgique, Comptes-Rendus des Séances 38 (2): 178 (De Wildeman \& Durand 1899). - Grewiella globosa (De Wild. \& T.Durand) T.Durand \& H.Durand, Sylloge Florae Congolanae: 70 (Durand \& Durand 1909). - Type: DEMOCRATIC REPUBLIC OF CONGO • Coquilhatville; 14 Jan. 1896; A.P. Dewèvre 614; lectotype: BR[BR0000008965608], isolectotype: BR[BR0000008963093] 2 sheets.

\section{Other material studied}

ANGOLA • Mayumbe; 22 Jan. 1916; J. Gossweiler 6182; BM • Cabinda, Buco Zau, close to Chiaca; $4^{\circ} 46^{\prime} \mathrm{S}, 12^{\circ} 33^{\prime} \mathrm{E}$; 20 Aug. 1958; R. Santos 213; BM.

CAMEROON • Campo Ma'an area, Ebodje, forest close to Likodo River mouth; $2^{\circ} 39^{\prime} \mathrm{N}, 9^{\circ} 51^{\prime} \mathrm{E} ; 8 \mathrm{Mar}$. 2001; T.R. van Andel 3236; WAG[WAG.1834962] • Bipindi; $3^{\circ} 04^{\prime}$ N, $10^{\circ} 25^{\prime}$ E; Jun. 1918; E. Annet 410; P Eseka, River Nyong km 12; 3³8' N, 1045' E; Dec. 1967; P. Bamps 1314; BR[BR0000020059811], $\mathrm{P} \cdot$ Forest Reserve of Kienke, Kribi-Ebolowa km 16; $2^{\circ} 55^{\prime} \mathrm{N}, 10^{\circ} 00^{\prime} \mathrm{E}$; Jan. 1968; P. Bamps 1684; BR[BR0000020059828], P - Bitye, Yaounde; 301' N, $12^{\circ}$ E, 1917; G.L. Bates 1668; BM, BR[BR0000020059798] • Bitya, near the River Ja; $3^{\circ} 01^{\prime} \mathrm{N}, 12^{\circ} 22^{\prime} \mathrm{E}$; G.L. Bates 1710 ; K $\bullet 6.5 \mathrm{~km} \mathrm{~S}$ of Kribi, Gr. Batanga road; 2०57' N, 955' E; 28 Oct. 1968; J.J. Bos 3170; BR[BR0000020059859], K, P, WAG[WAG.1834995] 2 sheets • about $12 \mathrm{~km}$ from Kribi, Lolodorf road; 259’ N, 959' E; 13 Jan. 1969; J.J. Bos 3628; BR[BR0000020059880], P - Lobé R. bank 1 km, above Gr. Batanga ferry; $2^{\circ} 52^{\prime} \mathrm{N}$, 954' E; 24 Jan. 1969; J.J. Bos 3754; BR[BR0000020059866], P • 18 km from Kribi, Lolodorf road; $3^{\circ} 00^{\prime} \mathrm{N}, 10^{\circ} 02^{\prime} \mathrm{E}$; 19 Mar. 1969; J.J. Bos 4162; BR[BR0000020059842], WAG[WAG.1845049] 2 sheets $\bullet 10 \mathrm{~km}$ from Kribi, Lolodorf road, N bank of Kienke River; $2^{\circ} 58^{\prime}$ N, $9^{\circ} 58^{\prime}$ E; 27 May 1969; J.J. Bos 4653; WAG[WAG.1845010] $20 \mathrm{~km}$ from Kribi, $2 \mathrm{~km}$ N of Lolodorf road; $3^{\circ} 01^{\prime} \mathrm{N}, 10^{\circ} 03^{\prime} \mathrm{E}$; 12 Dec. 1969; J.J. Bos 5804; P 40 km N of Kribi, $5 \mathrm{~km} \mathrm{E}$ of Edea road, forest track Fifinda-Bella; $3^{\circ} 13^{\prime} \mathrm{N}, 10^{\circ} 04^{\prime} \mathrm{E}$; 6 Feb. 1970; J.J. Bos 6264; WAG[WAG.1845015] • about $60 \mathrm{~km} \mathrm{~N}$ of Kribi; $3^{\circ} 19^{\prime} \mathrm{N}$, $10^{\circ} 06^{\prime} \mathrm{E}$; 13 Feb. 1970; J.J. Bos 6344; P • few km S of km 14 Kribi-Lolodorf; $2^{\circ} 58^{\prime}$ N, $10^{\circ} 01^{\prime} \mathrm{E}$; 4 Mar. 1970; J.J. Bos 6485; WAG[WAG.1845018] 2 sheets $113.5 \mathrm{~km}$ from Kribi, Ebolowa road; $2^{\circ} 51^{\prime} \mathrm{N}$, $10^{\circ} 00^{\prime}$ E; 29 Jun. 1970; J.J. Bos 6992; WAG[WAG.1845023] 2 sheets 4 km north of km 20 Kribi, Lolodorf; $3^{\circ} 02^{\prime} \mathrm{N}, 10^{\circ} 03^{\prime} \mathrm{E}$; 4 Jul. 1970; J.J. Bos 7019; P • $3 \mathrm{~km} \mathrm{~N}$ of Niète R., miner's road, SE of Kribi; $2^{\circ} 44^{\prime} \mathrm{N}, 10^{\circ} 03^{\prime} \mathrm{E}$; 30 Jul. 1970; J.J. Bos 7182; P - Lomié, near Catholic Mission; $3^{\circ} 09^{\prime} \mathrm{N}$, 13³8' E; 20 Apr. 1961; F.J. Breteler 1261; WAG[WAG.1845028] 3 sheets $\bullet$ near Dimako, between Bertoua and Doumé; $4^{\circ} 23^{\prime}$ N, 1334' E; 7 Dec. 1961; F.J. Breteler 2164; BR[BR0000020059835], K, WAG[WAG.1845033] 2 sheets $\bullet$ E of Méloundou, $50 \mathrm{~km} \mathrm{SW}$ of Batouri; $4^{\circ} 01^{\prime} \mathrm{N}, 1^{\circ} 06^{\prime} \mathrm{E} ; 17 \mathrm{Apr}$. 1962; F.J. Breteler 2843; WAG[WAG.1834990] 2 sheets - Bimbia; 358' N, 9¹5' E; 7 Mar. 1995; S. Cable 1442; K, WAG[WAG.1845035] • Onge, Enyenge; $4^{\circ} 17^{\prime} \mathrm{N}, 8^{\circ} 58^{\prime} \mathrm{E} ; 27$ Oct. 1993; M.R. Cheek

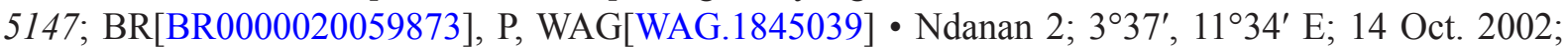
M.R. Cheek 11089; K・ Ndanan 1; $3^{\circ} 37^{\prime} \mathrm{N}, 1^{\circ} 34^{\prime} \mathrm{E} ; 15$ Mar. 2004; M.R. Cheek 11737; K • Yabassi;

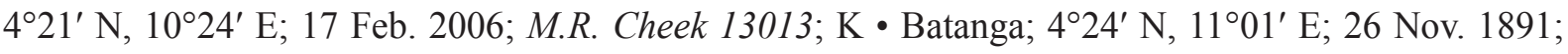
M.J. Dinklage 1409; P• Campo-Ma'an area, Bongola, along the Bongola River; $2^{\circ} 13^{\prime}$ N, $9^{\circ} 56^{\prime}$ E; 29 Jan. 
2000; M.E. Elad 1239; WAG[WAG.1845678] - Campo-Ma'an area, Medjivini, between Ekan and Ngo'ambang in Ma'an area; $2^{\circ} 17^{\prime} \mathrm{N}, 10^{\circ} 20^{\prime} \mathrm{E}$; 31 Mar. 2000; M.E. Elad 1440; WAG[WAG.1845679] - River Npolongwe, between Kribi and Londji; $2^{\circ} 57^{\prime}$ N, 955' E; 4 Mar. 1987; H. Huber 1044; P • Ngongondje hill, near Akonetye Village; $2^{\circ} 40^{\prime} \mathrm{N}, 12^{\circ} \mathrm{E} ; 28$ Aug. 1978; A. Koufani 119; K, P • foot of Ngongondje hill, near Akonetye, $2^{\circ} 30^{\prime}$ S of Ebolowa; 2 $40^{\prime} 12^{\prime \prime}$ N, 12 ${ }^{\circ} 52^{\prime} 12^{\prime \prime}$ E; 29 Aug. 1978; A. Koufani 141; P • Mount Kupe, Kupe Village, forest trail above Kupe Village; 446’ N, 942' E; 19 Jan. 1995; P. Lane 357; WAG[WAG.1834978] • left bank of Kélé R., 29 km N of Eséka; $3^{\circ} 50^{\prime}$ N, 1045' E; 30 Jun. 1965; A.J.M. Leeuwenberg 6022; WAG[WAG.1834984] 2 sheets • Kombite; 13 Jan. 1960; R. Letouzey 2639; BR[BR0000020059903], K, P • “Au nord du Moundi” [north of Moundi]; 4³4' N, 132 $28^{\prime}$ E; 16 Jan. 1960; R. Letouzey 2648; P “Au sud de Zingui" [south of Zingui], between the rivers Niete and Lobe; 2 ${ }^{\circ} 49^{\prime} \mathrm{N}, 10^{\circ} 58^{\prime} \mathrm{E}$; 20 Jan. 1962; $R$. Letouzey 4065; P • $5 \mathrm{~km} \mathrm{SW}$ of Nguila; 443’ N, $11^{\circ} 41^{\prime} \mathrm{E}$; 22 Dec. 1969; R. Letouzey 9760; BR[BR0000020059910], K, P, WAG[WAG.1834987] • near Njantibda,

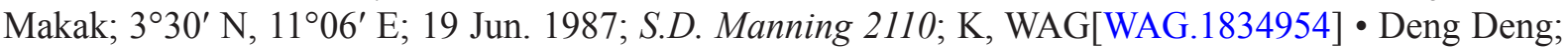
$5^{\circ} 10^{\prime} \mathrm{N}, 13^{\circ} 35^{\prime} \mathrm{E}$; Apr. 1914; G.W.J. Mildbraed 8866; K • ca $10 \mathrm{~km} \mathrm{~S}$ of Mekalat, between Mekalat and Lolodorf; $3^{\circ} 06^{\prime} \mathrm{N}, 10^{\circ} 44^{\prime} \mathrm{E}$; 20 Mar. 1996; A.S. Mutsaers 2; WAG[WAG.1834957] 2 sheets $\bullet$ Deng Deng; $5^{\circ} 11^{\prime} \mathrm{N}, 13^{\circ} 31^{\prime} \mathrm{E}$; 19 Jan. 1960; P. Nana 263; P • Mabeta-Moliwe; 359' N, 9॰15' E; 25 Nov. 1991; F. Nguembock 48; WAG[WAG.1845040] - Canon du Ntem; Mar. 1983; B.A. Nkongmenek 385; P

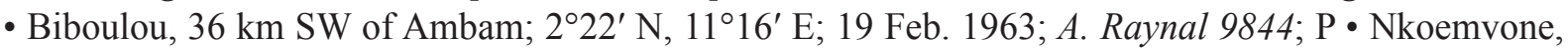

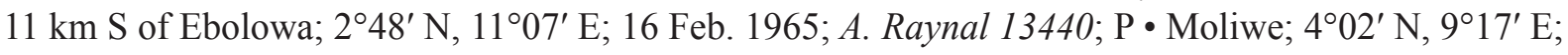
30 Jul. 1993; I. von Rege 29; K • Mabeta; 359' N, 9¹7' E; 11 Aug. 1993; I. von Rege 92; K • "Réserve de faune du Dja" [Dja Faunal Reserve], 24 km S of Djolimpoum; $3^{\circ} 20^{\prime} \mathrm{N}, 12^{\circ} 52^{\prime} \mathrm{E}$; 28 Feb. 1994; B. Sonké 971; BR[BR0000019963334] • "Réserve de faune du Dja" [Dja Faunal Reserve], $27 \mathrm{~km} \mathrm{~S} \mathrm{of}$ Djolimpoum; 3²0' N, 1252' E; 6 Mar. 1994; B. Sonké 1018; BR[BR0000020061074] • "Réserve de faune du Dja" [Dja Faunal Reserve], Djolimpoum; 3¹3' N, 1251' E; 13 Dec. 1994; B. Sonké 1337; BR[BR0000019963402] • Lolo Village; $3^{\circ} 14^{\prime}$ N, 1044' E; 1896; A. Staudt 211; K, P • Ndanan 1; 3³7' N, 11³5' E; 23 Mar. 2004; F. Tadjouteu 569; K, WAG[WAG.1845037] • ibid.; 27 Mar. 2004; F. Tadjouteu 590; K • Bechati; 540' N, 955' E; 24 Sep. 2006; B. Tchiengue 2766; K, WAG[WAG.1845036] • Campo Ma'an area, Bibabimvoto, in the National Park along Transect T4; $2^{\circ} 15^{\prime} \mathrm{N}, 10^{\circ} 15^{\prime} \mathrm{E} ; 24$ Aug. 2000; G.P. Tchouto Mbatchou T4X98; WAG[WAG.1845676] 2 sheets • Southwest Province, roadside forest $\mathrm{S}$ of Baro Village; $5^{\circ} 14^{\prime} \mathrm{N}, 9^{\circ} 15^{\prime} \mathrm{E}$; 31 Mar. 1988; D.W. Thomas 7495; WAG[WAG.1834963] - Campo Region, Dipikar island; 2¹3'57" N, 9०53'01" E; 10 Dec. 1998; J.J.F.E. de Wilde 12124; WAG[WAG.1845688] • $50 \mathrm{~km} \mathrm{NW}$ of Eséka, W of Yaoundé, on opposite site of the Kelè River; $3^{\circ} 50^{\prime} \mathrm{N}$, $10^{\circ} 27^{\prime}$ E; 22 Nov. 1963; W.J.J.O. de Wilde 1300; WAG[WAG.1845055] • ca 50 km NW of Eséka, W of Yaoundé; 339' N, 1046' E; 16 Dec. 1963; W.J.J.O. de Wilde 1508; P, WAG[WAG.1834980] • ca 40 km $\mathrm{S}$ of Badjob, ca $10 \mathrm{~km} \mathrm{~N}$ of the Njong-River, SW of Eséka; 3³9' N, 1046' E; 28 Jan. 1964; W.J.J.O. de Wilde 1723; BR[BR0000020059897], P, WAG[WAG.1845053] - ca $15 \mathrm{~km} \mathrm{~S}$ of Ebolowa; 3 ${ }^{\circ} 54^{\prime} \mathrm{N}$,

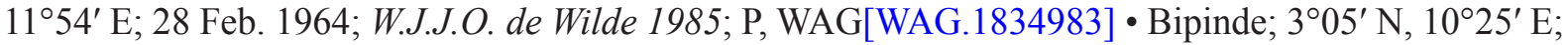
1896; G.A. Zenker 918; BM, E[E00934283] 2 sheets, HBG, K, P • ibid.; 1904; G.A. Zenker 3169; BM, BR[BR0000020059934] 2 sheets, E[E00934282], K, P•ibid.; G.A. Zenker 3705; WAG[WAG.1845031] 2 sheets.

CENTRAL AFRICAN REPUBLIC • Ngoto Forest; 401' N, 17²11' E; 3 Sep. 1994; L. Debroux 88; BR[BR0000020059972] • Dzanga-Sangha Reserve; $2^{\circ} 21^{\prime} \mathrm{N}, 16^{\circ} 10^{\prime} \mathrm{E} ; 10$ Oct. 1988; D.J. Harris 1387; $\mathrm{E}[\mathrm{E} 00930949] \bullet 45 \mathrm{~km} \mathrm{~S}$ of Lidjombo; $2^{\circ} 22^{\prime} \mathrm{N}, 16^{\circ} 10^{\prime} \mathrm{E}$; 4 Mar. 1989; D.J. Harris 1976; E[E00930952] - $45 \mathrm{~km} \mathrm{~S}$ of Lidjombo; $2^{\circ} 21^{\prime} \mathrm{N}, 16^{\circ} 09^{\prime} \mathrm{E}$; 11 Oct. 1990; D.J. Harris 2566; WAG[WAG.1845041] 2 sheets • Boukoko; 357' N, 1755' E; 14 Sep. 1951; Tisserant (Équipe) 2264; P • Boukoko; 354' N, 1755' E; 16 Feb. 1948; Tisserant (Équipe) 701; P.

CONGO • Zanaga Project; $2^{\circ} 58^{\prime} \mathrm{S}, 1^{\circ}{ }^{\circ} 34^{\prime} \mathrm{E}$; 12 Oct. 2009; M.R. Cheek 15710; K, WAG[WAG.1845038] - P.N. Odzala (Cuvette); 13 Oct. 1994; F. Dowsett-Lemaire 1805; BR[BR0000020060053] • Achoutha; 
22 Aug. 1895; M.J. Dybowski 125; P • "Chantier forestry of Mudongo" [logging camp of Mudongo], forest at Limba, $25 \mathrm{~km} \mathrm{~W}$ of Sibiti; $3^{\circ} 24^{\prime}$ S, $13^{\circ} 12^{\prime} \mathrm{E}$; 19 Aug. 1965; C. Farron 4497; P Les Saras, along N1; 421' S, 12²1' E; 13 Jan. 1987; H. de Foresta 1223; P • Sanghe; $1^{\circ} 33^{\prime}$ N, $16^{\circ} 17^{\prime}$ E; 14 Feb. 2007;

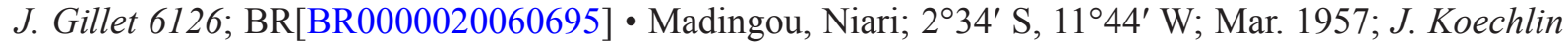
7905; P - Kouilou, Kakamoeka, left river bank of Kouilou; 4 $13^{\prime}$ S, $12^{\circ} 06^{\prime}$ E; 10 Jan. 1990; S. Lisowski B-8031; BR[BR0000020060046], WAG[WAG.1845046] • ibid.; 11 Oct. 1990; S. Lisowski B-8045; BR[BR0000020060060] - Simonbondo; $2^{\circ} 19^{\prime}$ S, 1340' E; 8 Oct. 2009; J.-.M. Moutsamboté 6484; K・ ibid.; 9 Oct. 2009; J.-.M. Moutsamboté 6527; K, WAG[WAG.1577666] • Bangou Forest; 356' S, 14²4' E; 23 Feb. 1960; P. Sita 203; P, WAG[WAG.1845737].

DEMOCRATIC REPUBLIC OF CONGO - Biaro; 0 ${ }^{\circ} 14^{\prime} \mathrm{N}, 2^{\circ} 19^{\prime} \mathrm{E} ; 30$ May 2009; C. Amani 651; BR[BR0000005773725] - Kisangani; 0³1' N, 25¹1' E; 9 Mar. 1915; J.C. Bequaert 7067; BR[BR0000008964946] 2 sheets • River Ikelemba; May 1913; P. Bonnivair 11; BR[BR0000020060572] 2 sheets • "Grotte de Binza" [Binza Cave]; 23 Jan. 1968; H. Breyne 469; BR[BR0000020060237] • ibid.; 23 Jan. 1968; H. Breyne 470; BR[BR0000020060220] • Mabana, left of road Menkao-Bombo River; 28 Feb. 1971; H. Breyne 2054; BR[BR0000020060251] • road Bita-Sualempu Terr. Maluku; 19 Mar. 1971; H. Breyne 2120; BR[BR0000020060244] - Mayumbe/Ganda Sundi; 2॰50' N, 276' E; Comte de Briey 176; BR[BR0000013288006] 3 sheets - low slopes of Namoya summit; $4^{\circ} 1^{\prime} \mathrm{S}, 27^{\circ} 33^{\prime} \mathrm{E}$; 13 Apr. 2008; B. Bytebier 2883; BR[BR0000005044887] - Muetshi $70 \mathrm{~km}$ WNW of Lusambo $20 \mathrm{~km}$ WZW of Bena Thiadi; 1982; P. Casier 293; BR[BR0000020061029] - Kasayi Muetshi; 23 Feb. 1983; P. Casier 413; BR[BR0000020061036] - Bokola; 1909; J. Claessens 124; BR[BR0000020060343] 2 sheets • Luba; 1921; J. Claessens 158; BR[BR0000020060596] 2 sheets • Kasangulu Chefferie; 8 Jun. 1960; P. Compère 2178; BR[BR0000020060275] - Prov. Kasai, Mweka, Kakenge; 21 Nov. 1958; R. Dechamps 78; BR[BR0000020060367], WAG[WAG.1845695] 2 sheets • Kiyaka-Kikwit-Kwango; 502' S, $18^{\circ} 5^{\prime}$ E; 9 Jul. 1955; R. Devred 1966; BR[BR0000020060350] • 1896; A.P. Dewèvre s.n.; P• Kiobo; $5^{\circ} 37^{\prime} \mathrm{S}, 13^{\circ} 13^{\prime} \mathrm{E}$; 23 Oct. 1945; C.A. Donis 371; BR[BR0000020060084],

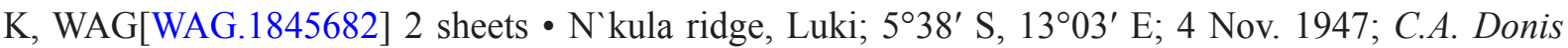
1528; BR[BR0000020060077], K・ Luki, Singa Valley; 5³8' S, 1303' E; 24 Sep. 1948; C.A. Donis 2007; BR[BR0000020060107] • ibid.; 24 Sep. 1948; C.A. Donis 2008; BR[BR0000020060091] • Luki, Kinkongo Valley; 5³8' S, 1303' E; 21 Oct. 1948; C.A. Donis 2071; BR[BR0000013287986] • Luki; 538' S, 1303' E; 27 Nov. 1948; C.A. Donis 2106; BR[BR0000013287993] - Kingana Forest; 24 Jun. 1958; J. Dubois 316; BR[BR0000020060268] • Prov. Equateur, Befale, Eandza; 19 Feb. 1958; C.M. Evrard 3509; BR[BR0000020060626], WAG[WAG.1845693] - BokoIngo-Djoa; 26 Feb. 1958; C. Evrard 3551; BR[BR0000020060619] • Djoa; 15 Oct. 1958; C. Evrard 5015; BR[BR0000020060633] - road Ekunga-Bosale; 2 ${ }^{\circ} 6^{\prime}$ N, 3046' E; 15 Aug. 1958; C. Evrard 4685; BR[BR0000020060640] • Yalisenga (Mondombe); $1^{\circ} 14^{\prime}$ N, 2244' E; 26 Dec. 1958; C. Evrard 5386; BR[BR0000020060664] • LuiKotale; $2^{\circ} 46^{\prime}$ S, $20^{\circ} 22^{\prime}$ E; Mar. 2003; B. Fruth 03/0985/b; M • Bokaw; $0^{\circ} 8^{\prime}$ S, $20^{\circ} 58^{\prime}$ E; 15 May 1954; $R$. Germain 8340; BR[BR0000020060688] • between Kwango-Lufuma, valley of Tsambi, village of Itambu; 3 Aug. 1944; R. Germain 2590; BR[BR0000020060381] • near Yapehe; $0^{\circ} 9^{\prime} \mathrm{S}, 2^{\circ} 20^{\prime} \mathrm{E}$; 15 Jun. 1949; R. Germain 4983; BR[BR0000020060657] - near Tumba Terre de Lodja Station Crête; 15 Aug. 1938; J. Gillardin 427; BR[BR0000020060701] - Sangaie; 499' S, 2351' E; Mar. 1939; J. Gillardin 538; BR[BR0000020060374] 2 sheets, K • near Kinshasa; 4¹8' S, 15¹8' E; 15 Aug. 1902; J. Gillet s.n.; BR[BR0000020060282] - Epulu, "Zone de Mambes" [area of Mambes], Ituri Forest; $1^{\circ} 25^{\prime} \mathrm{N}$, 2835' E; 16 Jul. 1991; T. Hart 1196; BR[BR0000020059569] • Luki; 5³8' S, 1303' E; 25 Oct. 1951; J. Hombert 2; BR[BR0000020060121] • ibid.; 21 Apr. 1951; J. Hombert 14; BR[BR0000020060114] - Dzelo "grot" [cave]; 4²3' S, 15¹5' E; 24 Mar. 1947; E. Jans 442; BR[BR0000013218003] • E. Jans s.n.; BR[BR0000013217990] - Bonga Village region of Ikela; 10 Jun. 1911; K. Jespersen s.n.; BR[BR0000020060725] - Wendji, near Coquilhotville; 0³' S, $18^{\circ} 9^{\prime} \mathrm{E}$; Aug. 1930; J.P.A. Lebrun 1012; BR[BR0000020060718] • Lumuna; Aug. 1932; J.P.A. Lebrun 5904; BR[BR0000020060732] 2 sheets, P • Ubundu; 0²1' S, 25²5’ E; 11 Mar. 1978; J. Lejoly 2876; BR[BR0000020060756] • 
Wangata-Watsiko at Ikoie, near Eala; 0 $4^{\prime}$ N, 18¹8' E; 1946; J. Léonard 418; BR[BR0000020060770] - Mabidi; 4¹7' S, 18²5' E; 20 Jun. 1903; E. Lescrauwaet 105; BR[BR0000020060398] • Yangole, $20 \mathrm{~km} \mathrm{~W}$ of Yangambi; $0^{\circ} 49^{\prime} \mathrm{N}, 2^{\circ} 16^{\prime} \mathrm{E}$; 22 Oct. 1938; J. Louis 11958; BR[BR0000020060800] 2 sheets - Yafalolame, between Opala and Mayoko; $0^{\circ} 43^{\prime}$ S, 235' E; 15 Feb. 1939; J. Louis 14224; BR[BR0000020060824] 3 sheets • 10 May 1890; R.E. Luja 269; BR[BR0000020060428] 3 sheets • Kikwit; 502' S, $18^{\circ} 48^{\prime}$ E; 7 Nov. 1990; B. Masens 161; BR[BR0000019963471], WAG[WAG.1845834] - E. Maudoux 135; BR[BR0000020060145] - Ruki; 122' N, 2422' E; 11 Oct. 1949; E. Maudoux 170; BR[BR0000020060138], K • "Grotte de Binza" [cave of Binza]; 23 Jan. 1968; F. Muambi 66; BR[BR0000020061050] • ibid.; 20 Feb. 1968; F. Muambi 117; BR[BR0000020060299] • Luki; $5^{\circ} 38^{\prime} \mathrm{S}, 13^{\circ} 03^{\prime} \mathrm{E} ; 17$ Jul. 1982; L. Nsimundele 1056; BR[BR0000020060169] - Ngenegene; 4 Oct. 1984; H.H. Ntahobavuka 4[5]; BR[BR0000020060961] - Kisangani, Ngenegene; 20 May 1985; H.H. Ntahobavuka 33; BR[BR0000020059149], WAG[WAG.1845706] • "Grotte de Binza" [cave of Binza]; 17 May 1967; L. Pauwels 5009; BR[BR0000020060312], WAG[WAG.1845056] • Minkudu, territory of Tshela; 338' S, $18^{\circ} 36^{\prime}$ E; 25 Oct. 1947; P. Toussaint 12; BR[BR0000020060152], K - Eala; $0^{\circ} 03^{\prime} \mathrm{N}, 18^{\circ} 18^{\prime}$ E; 1 Feb. 1907; L. Pynaert 1086; BR[BR0000020060848] 2 sheets - ibid.; 15 Oct. 1907; L. Pynaert 1767; BR[BR0000020060862] - Kikwit Forest; 502' N, 1849' E; 24 May 1946; M. Renier 2B; BR[BR0000020060459] - S of Booke, Monkoto National Park; 2०33' N, $22^{\circ} 0^{\prime}$ E; 15 May 1958; R. Robin 91; BR[BR0000020060855] - Lukolela; 24 Jul. 1959; L. Toka 226; BR[BR0000020060909] - Luki, valley of the N'tosi settlement at Terminalia; 5 ${ }^{\circ} 38^{\prime} \mathrm{S}, 13^{\circ} 03^{\prime} \mathrm{E}$; 14 Jan. 1948; L. Toussaint 149; BR[BR0000020060176] - Gimbi settlement at Terminalia in the Fuka Valley; 28 Oct. 1948; L. Toussaint 612; BR[BR0000020060329] - Pangu; 4 $17^{\prime} \mathrm{S}, 2^{\circ} 01^{\prime} \mathrm{E}$; 15 Jun. 1920; H. Vanderyst 9556; BR[BR0000020060466] - Ipamu, Kikwit; 4 $9^{\prime}$ S, $1^{\circ} 38^{\prime}$ E; 1921; H. Vanderyst 9936; BR[BR0000020060497] 2 sheets - Kamtshar; 343' S, 1855' E; 1921; H. Vanderyst 10130; BR[BR0000020060534] - Mpio; 4¹3' S, 19³8' E; 1921; H. Vanderyst 10236; BR[BR0000020060527] • Ipamu; 49' S, 19³8' E; 1921; H. Vanderyst 10597; BR[BR0000020060558] - ibid.; 1 Jul. 1922; H. Vanderyst 12232; BR[BR0000020060541] - Kangu; 5¹5' S, 1256' E; 12 Oct. 1930; H. Vanderyst 26299; BR[BR0000020060190] - Temvo; 5²9' S, 1303' E; 25 Feb. 1919; F. Vermoesen 1662; BR[BR0000020060183] • Eala; $0^{\circ} 03^{\prime} \mathrm{N}, 18^{\circ} 18^{\prime} \mathrm{E} ; 2$ May 1919; F. Vermoesen 2127; BR[BR0000020060893] 3 sheets • ibid.; 15 May 1919; F. Vermoesen 2261; BR[BR0000020060947] 3 sheets - Ineac-Luki; 538' S, 1304' E; 7 Apr. 1959; J. Wagemans 2282; BR[BR0000020060213], WAG[WAG.1845045] • Bokondji; 2 Jul. 1959; P. de Wanckel 121; BR[BR0000020060602] • Vaku; $5^{\circ} 18^{\prime} \mathrm{S}, 13^{\circ} 15^{\prime} \mathrm{E}$; Nov. 1923; F. Wellens 445; BR[BR0000020060206].

EQUATORIAL GUINEA • Region Continental; 28 Jul. 1999; F. Eneme Efua 446; WAG[WAG. 1845680] - Mabungo 173' N, 1064' E; 25 Apr. 1908; G. Tessmann 378; K • Vega Ersatrek; 14 Sep. 1908; G. Tessmann 556; K.

GABON • Nyanga, road Tchibanga-Ndende; $2^{\circ} 58^{\prime} \mathrm{S}, 11^{\circ} 06^{\prime}$ E; 25 Oct. 2009; P. Bissiengou 527; BR[BR0000020060008] - Nyanga, Mourindi; $2^{\circ} 34^{\prime}$ S, $10^{\circ} 45^{\prime}$ E; 15 Sep. 2000; H.P. Bourobou 233; BR[BR0000009218185], P • Monts de Cristal, along the Mbé River; 050' N, 10³0' E; 23 Aug. 1978; F.J. Breteler 247; BR[BR0000020059958], WAG[WAG.1845021] 2 sheets • Moyen-Ogooué, ca 20$30 \mathrm{~km}$ NNW of Ndojé; 0³' S, 1045' E; 1 Oct. 1994; F.J. Breteler 13116; BR[BR0000020060022], K, WAG[WAG.1845689] 2 sheets - Haut-Ogooué, S of Bambidie; $0^{\circ} 42^{\prime} \mathrm{S}, 1^{\circ} 00^{\prime} \mathrm{E} ; 7$ Oct. 1997; F.J. Breteler 14236; BR[BR0000020059989] - Adouma, at Orimbo, Ogooué; 040' S, 10¹3' E; 29 Jul. 1912; F. Fleury 26229; K, P, WAG[WAG.1845022] - around Nkogo at the Ogooué; $0^{\circ} 16^{\prime}$ N, $9^{\circ} 15^{\prime}$ E; 14 Aug. 1912; F. Fleury 26350; P - Station d'Ipassa, $10 \mathrm{~km} \mathrm{~S}$ of Makokou; $0^{\circ} 34^{\prime} \mathrm{N}, 12^{\circ} 53^{\prime} \mathrm{E}$; 28 Apr. 1978; J. Florence 1110; P • La Nkoulounga; 9 Jul. 1959; N. Hallé 740; P • Abanga; 4 Jun. 1963; N.Hallé 2215; P • Belinga; $1^{\circ} 05^{\prime} \mathrm{N}, 13^{\circ} 11^{\prime} \mathrm{E} ; 13$ Nov. 1946; N. Hallé 3168; P • Nkogo; 15 Aug. 1912; E. Jablonszky 26360; P・11 Sep. 1901; T.-J. Klaine 2381; K, P・Libreville; 0²2' N, 9²6’ E; 14 Aug.

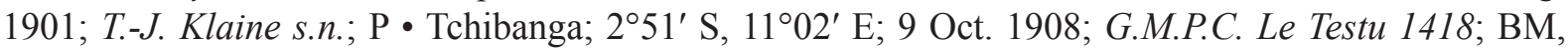


BR[BR0000020060039], K, P • Gabon River; Jul. 1861; G. Mann 992; K • "Mont Fene, inselberg au pied du village d'Efôt, à $15 \mathrm{~km}$ de Médouneu en direction de Sam" [Mont Fene, inselberg at foot of village Efôt, at $15 \mathrm{~km}$ of Médouneu in direction of Sam]; $1^{\circ} 00^{\prime} \mathrm{N}, 10^{\circ} 54^{\prime} \mathrm{E} ; 15 \mathrm{Jan} .2000$; I. Parmentier 714; WAG[WAG.1845043] • Ogooué-Maritime; $2^{\circ} 03^{\prime}$ S, $10^{\circ} 28^{\prime}$ E; 14 Nov. 2005; M.S.M. Sosef 2278; BR[BR0000020061067], K, WAG[WAG.1834960] 2 sheets • Mission St-Martin, Ngounie; 1938; A.Walker s.n.; P 26 km ENE of Lambaréné, 6 km ENE of Bellevue; 0³5' S, 10²6' E; 2 Apr. 1994; J.J. Wieringa 2622; WAG[WAG.1845685] - about $25 \mathrm{~km} \mathrm{SW}$ of Doussala; $2^{\circ} 25^{\prime} \mathrm{S}, 10^{\circ} 33^{\prime} \mathrm{E}$; 26 Nov. 1986; J.J.F.E. de Wilde 8961; BR[BR0000020059996], K, P, WAG[WAG.1845686] 2 sheets • ca $20 \mathrm{~km}$ SSE of Doussala; 2²5' S, $10^{\circ} 43^{\prime}$ E; 15 Mar. 1988; J.J.F.E. de Wilde 9362; BR[BR0000020059965], WAG[WAG.1845048] 2 sheets.

GHANA • Asinanyo River Forest Reserve; Feb. 1937; J.E. Andoh 4300; K • Bobiri Forest Reserve; Jan. 1951; J.E. Andoh FH5457; BR[BR0000020059750], K, P • Bobiri Forest Reserve Juaso, Ashanti; 19 Jan. 1972; A.A.Enti Sp537; BR[BR0000020059781] • between 6-8 km NW of intersection of Accra-Kumasi road at Sagyimase along forest access road, Atewa Range, Forest Reserve; $6^{\circ} 13^{\prime} \mathrm{N}, 0^{\circ} 32^{\prime} \mathrm{W}$; 4 Jul. 1995; D.K. Harder 3327; WAG[WAG.1845057] - W.A.C.R.I., Tafo; $6^{\circ} 44^{\prime} \mathrm{N}, 1^{\circ} 36^{\prime} \mathrm{W}$; Jul. 1961; F.R. Irvine 4979; K・ ibid.; 2 Nov. 1953; N.K.Lovi WACRI3844; K, P・1941; J. Scholles 165; K・ Gabo, upper Wassow Reserve; 11 Feb. 1927; C. Vigne 279; BM, K • C. Vigne 1044; BM, BR[BR0000020059774], K • Kwaku Prasu; Feb. 1929; C. Vigne 1599; K・ South Fomany Su Reserve; 1930; C. Vigne 1831; K.

GUINEA • Nimba Mountains, "Forêt de Gouan" [Gouan Forest]; 7²4' N, 8²3' W; 22 Dec. 2008; O.O. Haba 76; BR[BR0000005091423], WAG[WAG.1845059].

IVORY COAST • A. Aubréville 1217; P• Guiglo, Zaipobly; $7^{\circ} 29^{\prime}$ N, $5^{\circ} 57^{\prime}$ W; 27 Jan. 2001; A. Bakayoko 22; P •"Soubré Forêt Classée" [Soubré Forest]; 540' N, 6²0' W; Feb. 1969; P. Bamps 2092; K, P, WAG[WAG.1834988] • Pinhou; 6³7' N, 7¹9' W; Feb. 1969; P. Bamps 2136; BR[BR0000020059743], K • ibid.; 20 Mar. 1969; P. Bamps 2241; BR[BR0000020059736] - Tienkula; 607' N, 7²9' W; L. Bernardi 8356; K, P, WAG[WAG.1845684] • "Env. de Bingerville" [surroundings of Bingerville]; Apr. 1913; N. de Byans s.n.; L[L.2349654] • Bouroukrou; 740' N, $3^{\circ} 12^{\prime}$ W; 27 Dec. 1906; A.J.B. Chevalier 16697; K, P • Bomoukrou, railway km 92; Jan. 1907; A.J.B. Chevalier 16708; P • Bouroukrou; $7^{\circ} 40^{\prime} \mathrm{N}$, $3^{\circ} 12^{\prime}$ W; 5 Jan. 1907; A.J.B. Chevalier 16837; K, P, WAG[WAG.1845062] • N of Trouvougbeu, $6 \mathrm{~km}$ from village; $7^{\circ} 18^{\prime} \mathrm{N}, 7^{\circ} 01^{\prime} \mathrm{W}$; $10 \mathrm{Feb}$. 1995; L. Gautier LG2606; BR[BR0000020059712] • on bank of Sassandra River, near Louga; $5^{\circ} 03^{\prime} \mathrm{N}, 6^{\circ} 13^{\prime} \mathrm{W}$; sandy soil; 9 Apr. 1973; J. de Koning 1344; BR[BR0000020059705], WAG[WAG.1845060] 2 sheets • behind Fuyt Plantation; 503' N, 6 ${ }^{\circ} 14^{\prime}$ W; 12 Nov. 1973; J. de Koning 2678; BR[BR0000020059699], WAG[WAG.1845070] 2 sheets $\bullet 61 \mathrm{~km} \mathrm{~N}$ of Sassandra; 5¹9' N, 610' W; 19 Feb. 1959; A.J.M. Leeuwenberg 2759; BR[BR0000020059767], K, L[L.2349653], P, WAG[WAG.1845065] 2 sheets • ca $16 \mathrm{~km} \mathrm{NW}$ of Sassandra, ca $4 \mathrm{~km} \mathrm{SE} \mathrm{of} \mathrm{Louga,}$ W of Sassandra River; 500' N, 6²' W; 15 Jun. 1963; W.J.O. de Wilde 233; K, WAG[WAG.1845692] 3 sheets - "Region d' Abidjan" [region of Abidjan], "K.P. 64"; 22 Aug. 1956; J.J.F.E. de Wilde 376; WAG[WAG.1845067].

LIBERIA - National Forest, 18 miles N of Tapeta; $6^{\circ} 45^{\prime}$ N, $8^{\circ} 52^{\prime}$ W; 22 Feb. 1961; A.G. Voorhoeve 198; BR[BR0000020059682], WAG[WAG.1845063] 2 sheets.

NIGERIA • Sapoba Forest Reserve; $6^{\circ} 06^{\prime}$ N, 553' E; Jan. 1935; FHI1259; K • Okomu Forest Reserve; $6^{\circ} 15^{\prime}$ N, 506' E; 25 Dec. 1947; J.P.M. Brenan 8615A; BM, K, P - Akamkpa, Gmeling Plantation; $5^{\circ} 18^{\prime}$ N, 8²1' E; 16 Feb. 1993; B.O. Daramola 18; K • Okomu Forest Reserve; 20 Feb. 1953; M.C. Ejiofor FHI19741; K • North Onda Enclave village along the Onda-Owena stream path; 27 Feb. 1946; A.P.D. Jones 15373; K - Afi River Forest Reserve, on path from Boje to Iso Bendiga; $6^{\circ} 17^{\prime} \mathrm{N}$, 855' E; 14 Dec. 1950; R.W.J. Keay FHI28254; BR[BR0000020059804], K • J.D. Kennedy 1958; K 
- Sapoba; 606' N, 553' E; J.D. Kennedy 2349; K - Akampka rubber estate, Calabar River; 518' N, $8^{\circ} 21^{\prime}$ E; 14 Mar. 1959; M.G. Latilo FHI41333; K • Oban Forest Reserve, Orem; 519' N, 8³4' E; 25 Jan. 1957; J.C. Okafor FHI36156x; K・ Iguobazowa Forest Reserve; 6³3' N, 5²1' E; 24 Jan. 1961; J. Olorunfemi FHI41469; K • Ikeji-Ipetu Forest Reserve; 7²6' N, $4^{\circ} \mathrm{E}$; Mar. 1969; J. Olurunfemi FHI20538; K, P• Okumu Forest Reserve; 6¹5' N, $5^{\circ} 06^{\prime}$ E; 5 Jan. 1948; C.F.A. Onochie 8810; K • Okomu Forest Reserve, along main road; $6^{\circ} 15^{\prime} \mathrm{N}, 5^{\circ} 06^{\prime} \mathrm{E} ; 19$ Feb. 1953; C.F.A. Onochie FHI19730; K • Oban; $5^{\circ} 19^{\prime} \mathrm{N}, 8^{\circ} 34^{\prime} \mathrm{E} ; 1911 ;$ P.A. Talbot 443; BM • Oban; 5¹9' N, 834' E; 1909; P.A. Talbot 642; BM • Oban District; P.A. Talbot 1430; K • near Etemi Odo, Omo Reserve; 20 Nov. 1946; A. Tamajong FHI20744; K, P • Okomu Forest Reserve; 6¹9' N, 5²0' E; 3 Mar. 1997; Verwilghen 22; BR[BR0000019964119], WAG[WAG.1845720].

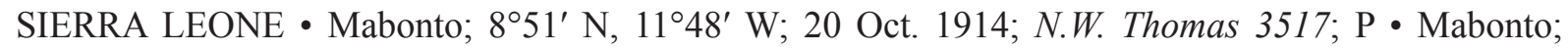
$8^{\circ} 51^{\prime} \mathrm{N}, 11^{\circ} 48^{\prime} \mathrm{W}$; 20 Oct. 1914; N.W. Thomas 3525; K.

\section{Description}

HAвіт. Small tree or shrub, to $2-3(-8) \mathrm{m}$ tall, $\mathrm{DBH}$ to $2-10 \mathrm{~cm}$, branches and twigs densely red-brown pubescent to glabrous.

LEAVES. With 3-5-fid stipules, finely divided into linear to filiform lobes, 4-9 $\mathrm{mm}$ long, hirsute; petiole distinctly inflated in the upper part, reddish-brown tomentose, $0.5-1.8 \mathrm{~cm}$ long; leaf blades usually drying green, oblong-oblanceolate to oblong-elliptic, papery, 13.5-25(-34) cm long and 4.5-9.5(-11) cm wide, glabrous apart from sparsely pubescent veins above, puberulous to pubescent with scattered stellate hairs below, secondary veins often looping, apex distinctly acuminate, base cordate to subcordate and sometimes asymmetric, margin obscurely toothed or with very few, small, acute, forward-pointing teeth; sometimes domatia formed of long hairs in the axils of the lateral veins.

INFLORESCENCE. An axillary or terminal cyme of 3-7 flowers; loose and slender; peduncle $0.5-4.5 \mathrm{~cm}$ long, pubescent; involucral bracts, either 3-4-fid, 4-6 mm long, lobes linear to filiform, hirsute, usually persistent, or lanceolate, 4-5 mm long, brownish-tomentose, falling off early, none enveloping the flower buds.

FLOWERS. pedicellate; pedicel 4-10 mm long, pinkish-tomentose; buds ovoid, pinkish-velvety; sepals oblong to oblanceolate, $8-10 \mathrm{~mm}$ long and $2-3 \mathrm{~mm}$ wide, borders fringed at the apex, tomentose; petals oblong, rounded at the apex, 2-3 mm long, $1-1.5 \mathrm{~mm}$ wide; ovary 5-7-locular.

Fruits. Oblong-elliptic, flattened at base and apex, $6.5-8 \mathrm{~cm}$ long and 5-6 cm wide, grooved, glabrous.

SeEds. Obovoid, $1.8 \mathrm{~cm}$ long, $8 \mathrm{~mm}$ wide.

Distribution (see Fig. 9)

Angola, Cameroon, Central African Republic, Congo, Democratic Republic of Congo, Equatorial Guinea, Gabon, Ghana, Guinea, Ivory Coast, Liberia, Nigeria, Sierra Leone.

\section{Habitat}

Terra firma forest, understory, fruiting and flowering in full shade. At altitudes of 250-1025 m a.s.1.

\section{Phenology}

Flower: March, August, October, fruit: January, Juny-August. 


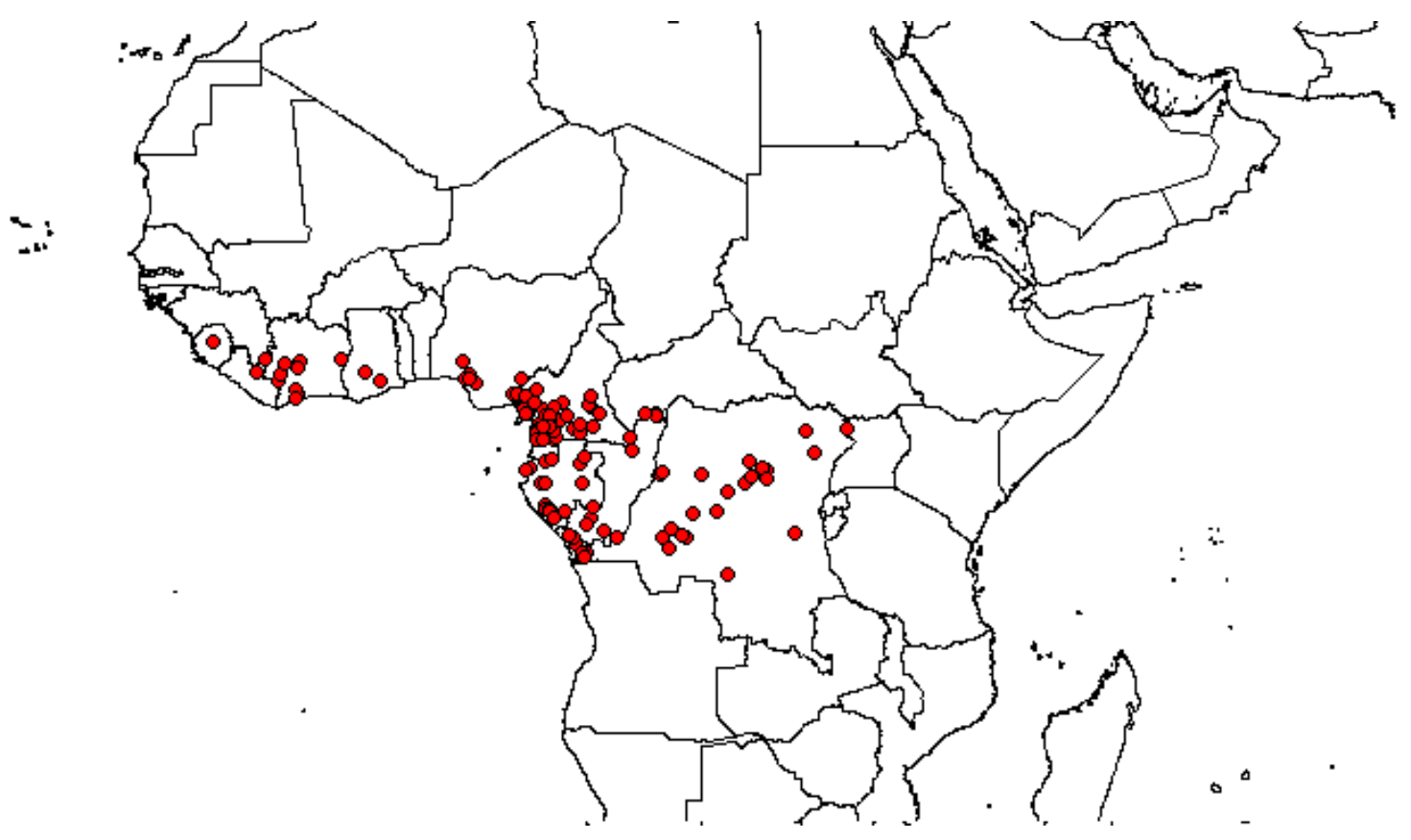

Fig. 9. Distribution map for Desplatsia subericarpa Bocq.

\section{Proposed IUCN status}

Least Concern (LC). Desplatsia subericarpa has an EOO of $4062478 \mathrm{~km}^{2}$ and an AOO of $580 \mathrm{~km}^{2}$, with a wide distribution across the forests of West and Central Africa. There are no major threats. Several collections are from protected areas which give this species some level of protection. Our assessment as LC is in agreement with the IUCN Red List assessments of threatened species (BGCI and IUCN SSC Global Tree Specialist Group 2019b).

\section{Notes}

A specimen of Mann 1695 at $\mathrm{K}$ was chosen by us from all the sheets of this number at $\mathrm{K}$ and $\mathrm{P}$ as the lectotype, because it also includes fruits.

The specimens Zenker 918 (BM, E, HBG, K, P) were annotated by Pellegrin (1924) as Grewia oligantha K.Schum., later corrected by himself to D. subericarpa. Grewia oligantha K.Schum. has, to the best of our knowledge, never been effectively published.

Five specimens do not entirely conform to the species description, but are tentatively placed under this species: Donis 1528 (BR, P), Manning 2110 (K, WAG), Masens 161 (BR, WAG) Maudoux 170 (BR, K), and Toussaint 12 (BR, K). Some are unusually hairy and some dry brown, characters usually associated with $D$. chrysochlamys, which is why we first thought of some as D. chrysochlamys. However, the leaf tip is distinctly acuminate and the leaf margin only shows very few, small, sharp teeth, characters indicating that the specimens are D. subericarpa.

\section{Other names}

Pleianthemum macrophyllum K.Schum. ex A.Chev.

Exploration botanique de l'Afrique occidentale française. Vol. 1: Énumération des Plantes: 92 (Chevalier 1920) nom. inval.; no description. 


\section{Excluded names}

Desplatsia caudata Pierre ex A.Chev. syn. nov.

Les végétaux utiles de l'Afrique tropicale française. Etudes scientifiques et agronomiques. Vol. 9: 81 (Chevalier 1917). - Type: GABON - Libreville; $0^{\circ} 22^{\prime}$ N, 9²7' E; C.J. Spire 44; holotype: P[MNHN-P-P04694148].

We have identified the specimen as Duboscia macrocarpa Bocq. and suggest Desplatsia caudata Pierre ex A.Chev. as a new synonym for Duboscia macrocarpa Bocq.

\section{Discussion}

Of the six species recognized by Burret (1926) (D. subericarpa, D. chrysochlamys, D. dewevrei, $D$. mildbraedii, $D$. floribunda syn. nov. and $D$. trillesiana syn. nov.) four are recognized here: D. subericarpa, D. chrysochlamys, D. dewevrei and D. mildbraedii.

Twelve names are treated by us as synonyms, two of which have been put into synonymy for the first time: D. floribunda syn. nov. and D. trillesiana syn. nov. Other names from literature were found to be invalid, illegitimate, or not effectively published; one name (D. caudata) was excluded as it belongs in another genus.

The characteristics and distribution ranges of the four Desplatsia species recognized here have been clarified and a key to their determination has been provided.

The large number of synonyms is due to a historical misunderstanding of certain characters. The genus Grewiopsis was described as being different from Desplatsia by having an ovary with 10 locules rather than 5 locules (De Wildeman \& Durand 1899), a character that does not hold. Desplatsia trillesiana syn. nov. and $D$. dewevrei were also erroneously distinguished as two species solely on the grounds of the number of locules in the ovary, despite them being recognized as very similar already when first describing $D$. trillesiana syn. nov. The genus Ledermannia was historically differentiated from Desplatsia by its large, round, undivided bracts around the inflorescence (Mildbraed 1912). Only when Burret (1926) described D. floribunda syn. nov., which also showed the presence of undivided bracts, was Ledermannia recognized as part of Desplatsia and the emphasis on bractmorphology reduced as a character at the genus level.

The ranges of the four Desplatsia species were mapped for the first time in such detail with more information than the previous maps (Lebrun \& Stork 2003). The ranges of D. chrysochlamys, D. dewevrei and $D$. subericarpa (see Figs 5, 7,9) fit in with earlier observed phytogeographical patterns (White 1979). White (1979) has described three phytogeographical regions: the Upper Guinean subcentre of endemism, the Lower Guinean subcentre of endemism and the Congolian subcentre of endemism, with the Upper Guinean subcentre of endemism normally being divided from the other two zones by the "Dahomey Gap". The "Dahomey Gap" is seen for D. subericarpa and D. dewevrei. For D. chrysochlamys, a "Cross River Terminus" distribution pattern (White 1979) is observed with no collections being made in Nigeria. Since D. chrysochlamys is a very distinctive tree one can assume that the observed pattern is real and not a result of under-collecting in Nigeria. Similarly we expect the absence from most of Gabon of $D$. chrysochlamys to be real, however the single record very close to the border with Equatorial Guinea indicates that the species is probably present at other localities in the north of Gabon. The distributional range for $D$. mildbraedii (see Fig. 8) clearly differs from that of the other three Desplatsia species, since it is only found along the northern edge of the Congo basin. D. mildbraedii appears to have a disjunct distribution. Although collections of $D$. dewevrei and $D$. chrysochlamys have been made 
in the north of the Democratic Republic of Congo, the lack of D. mildbraedii collections in that area does not necessarily mean that $D$. mildbraedii is absent from the area. It might still be an artefact of under-collecting in the north of the Democratic Republic of Congo, if D. mildbraedii is present there but rarer than D. dewevrei and D. chrysochlamys as is the case in SE Cameroon and SW Central African Republic. We accept that this might be a real disjunction but think it is more likely that it is due to undercollecting.

The wide distributions for Desplatsia species over West and Central Africa may be partly due to the dispersal of the fruits by elephants, which was noted on the labels of all species except $D$. mildbraedii, but is mentioned in the protologue for D. mildbraedii by Burret (1926). Since, however, elephant dispersal is not obligate, and dispersal of Desplatsia has been reported in areas with no elephants (Hawthorne 1995), the picture of the way of distribution is not fully clear.

Since the specimens examined cover a large area, there is currently no urgent need for additional Desplatsia collections, other than additional collections for $D$. mildbraedii.

\section{Acknowledgements}

The curators of BM, BR, K, M and P are thanked for permission to work in their herbaria. Xander van der Burgt (K) and Jan Wieringa (WAG) helped with the provision of electronic specimen data. Martin Cheek was our host at K, Mark Carine at BM, Ann Bogaerts and Sophie de Smedt at BR and Germinal Rouhan at P. Siegfried Springer (M) is thanked for sending scans of herbarium material. Victoria Stewart is thanked for testing the Desplatsia key. Rosemary Wise allowed her drawings to be reused and Sanna Olander prepared additional illustrations. Carel Jongkind is thanked for providing photographs of D. chrysochlamys and Gilles Dauby is thanked for providing a photograph of D. subericarpa. William Hawthorne provided information on D. chrysochlamys. Sanna Olander is thanked for help with GeoCAT and Robyn Drinkwater is thanked for an introduction to the digital microscope camera. Tanja Weibulat helped with submission of our data to the GFBio portal. We thank the editors, Frederik Leliaert, and two anonymous reviewers for constructive comments and additional references to relevant literature.

\section{References}

Bachman S., Moat J., Hill A.W., Torre J. de la \& Scott B. 2011. Supporting Red List threat assessments with GeoCAT: Geospatial conservation assessment tool. ZooKeys 150: 117-126.

https://doi.org/10.3897/zookeys.150.2109

Bayer C. \& Kubitzki K. 2003. Malvaceae. In: Kubitzki K. \& Bayer C. (eds) The Families and Genera of Vascular Plants, Flowering Plants, Dicotyledons 5: 225-311. Springer Press, Berlin, Heidelberg.

Bocquillon H. 1866. Mémoire sur le groupe des Tiliacées. Adansonia 7: 17-64.

Botanic Gardens Conservation International (BGCI) \& IUCN SSC Global Tree Specialist Group. 2019a. Desplatsia dewevrei. The IUCN Red List of Threatened Species 2019: e.T144289483A149034810. https://doi.org/10.2305/IUCN.UK.2019-2.RLTS.T144289483A149034810.en

Botanic Gardens Conservation International (BGCI) \& IUCN SSC Global Tree Specialist Group. 2019b. Desplatsia subericarpa. The IUCN Red List of Threatened Species 2019: e.T143711191A143711193. https://doi.org/10.2305/IUCN.UK.2019-1.RLTS.T143711191A143711193.en

Brunken U. \& Muellner A.N. 2012. A new tribal classification of Grewioideae (Malvaceae) based on morphological and molecular phylogenetic evidence. Systematic Botany 37 (3): 699-711.

https://doi.org/10.1600/036364412X648670 
Burret M. 1926. Beiträge zur Kenntnis der Tiliaceen. Notizblatt des botanischen Gartens und Museums zu Berlin-Dahlem 9: 592-880.

Cable S. \& Cheek M. 1998. The Plants of Mount Cameroon: A Conservation Checklist. Royal Botanic Gardens, Kew.

Cheek M., Harvey Y. \& Onana J.M. 2011. The Plants of Mefou Proposed National Park, Yaounde, Cameroon: A Conservation Checklist. Royal Botanic Gardens, Kew.

Cheek M., Pollard B., Darbyshire I., Onana J. \& Wild C. 2004. The Plants of Kupe, Mwanenguba and the Bakossi Mountains, Cameroon: A Conservation Checklist. Royal Botanic Gardens, Kew.

Chevalier A. 1912. Novitates florae Africanae. Bulletin de la Société botanique de France 58 (Memoire 8d): $137-224$.

Chevalier A. 1917. Les végétaux utiles de l'Afrique tropicale française. Etudes scientifiques et agronomiques. Vol. 9: La forêt et les bois du Gabon. Paris.

Chevalier A. 1920. Exploration botanique de l'Afrique occidentale française. Vol. 1: Énumération des Plantes. Paul Lechevalier, Paris.

Chung R.C.K.\& Soepadmo E. 2011. Taxonomic revision of the genus Microcos (Malvaceae-Grewioideae) in Peninsular Malaysia and Singapore. Blumea - Biodiversity, Evolution and Biogeography of Plants 56 (3): 273-299. https://doi.org/10.3767/000651911X619704

Crisp M.D. \& Weston P.H. 1993. Geographic and ontogenetic variation in morphology of Australian waratahs (Telopea: Proteaceae). Systematic Biology 42 (1): 49-76. https://doi.org/10.1093/sysbio/42.1.49

De Wildeman E.A.J. 1903. Annales du Musée du Congo belge. Série 5 Botanique. Vol. 1: Etudes de Systématique et de Géographie botanique sur la Flore du Bas- et du Moyen-Congo. Brussels.

De Wildeman E.A.J. \& Durand T. 1899. Matériaux pour la Flore du Congo. Bulletin de la Société royale de Botanique de Belgique. Comptes-Rendus des Séances 38 (2): 171-220.

Durand T. \& Durand H. 1909. Sylloge Florae Congolanae. Maison A. de Boeck, Brussels.

Hall J.B. \& Swaine M.D. 1981. Distribution and Ecology of Vascular Plants in a Tropical Rain Forest. Forest Vegetation in Ghana. Geobotany 1., Dr. W. Junk Publishers, The Hague/Boston/London.

Harris D.J. 2002. The Vascular Plants of the Dzanga-Sangha Reserve, Central African Republic. National Botanic Garden of Belgium, Meise.

Harris D.J. \& Wortley A.W. 2008. Sangha Trees. Royal Botanic Garden Edinburgh, Edinburgh.

Harris J.G. \& Woolf Harris M. 1994. Plant Identification Terminology, Second Ed. Spring Lake Publishing, Spring Lake.

Harris D.J. \& Wellsow J. 2019. A taxonomic revision of the African genus Desplatsia Bocq. (MalvaceaeGrewioideae) with identifiers. Dataset: v4; publisher: Dryad. https://doi.org/10.5061/dryad.j6q573n89

Hawthorne W. 1990. Field Guide to the Forest Trees of Ghana. Ghana Forestry Series 1, Natural Resources Insitute for the Overseas Departments Administration, Chatham.

Hawthorne W. 1995. Ecological Profile of Ghanaian Forest Trees. Tropical Forestry Paper No. 29. Oxford Forestry Institute, Department of Plant Science, University of Oxford, Oxford. 
Hijmans R.J., Schreuder M., De La Cruz J. \& Guarino L. 1999. Using GIS to check co-ordinates of genebank accessions. Genetic Resources and Crop Evolution 46 (3): 291-296.

https://doi.org/10.1023/A:1008628005016

Hutchinson J. \& Dalziel J. 1927. Flora of West Tropical Africa, First Ed. The Crown Agents for the colonies, London.

Hutchinson J. \& Dalziel J. 1954. Flora of West Tropical Africa, Second Ed. Vol. 1, 2. Crown Agents for Overseas Governments Administrations, London.

Hyam R., Drinkwater R.E. \& Harris D.J. 2012. Stable citations for herbarium specimens on the internet: An illustration from a taxonomic revision of Duboscia (Malvaceae). Phytotaxa 73: 17-30.

https://doi.org/10.11646/phytotaxa.73.1.4

Irvine F. 1961. Woody Plants of Ghana. Oxford University Press, London.

IUCN 2012. IUCN Red List Categories and Criteria: Ver. 3.1, Second Ed. IUCN, Gland/Cambridge.

Keay R.W.J. 1989. Trees of Nigeria. Clarendon Press, Oxford.

Kuntze C.E.O. 1903. In: Post T.E. von \& Kuntze C.E.O. (eds) Lexicon Generum Phanerogamarum. Deutsche Verlags-Anstalt, Stuttgart.

Lebrun J.-P. \& Stork A. 1997. Énumération des plantes à fleurs d'Afrique tropicale. Conservatoire et Jardin botaniques de la Ville de Genève, Geneva.

Lebrun J.-P. \& Stork A. 2003. Tropical African Flowering Plants: Ecology and Distribution. Vol. 1: Annonaceae-Balanitaceae. Edition des Conservatoire et Jardin botanique, Geneva.

Mildbraed J. 1912. Wissenschaftliche Ergebnisse der Deutschen Zentral-Afrika-Expedition, 1907-1908, unter Führung Adolf Friedrichs, Herzogs zu Mecklenburg. Vol. 2: Botanik. Klinghardt und Biermann, Leipzig.

Pellegrin F. 1924. La flore du Mayombe: d'après les récoltes de M. Georges Le Testu. Memoire de la Société linnéenne de Normandie XXVI (11), Société linnéenne de Normandie, Paris.

Sosef M.S.M., Wieringa J.J., Jongkind C.C.H., Achoundong G., Azizet Issembé Y., Bedigian D., Berg R.G. van den, Breteler F.J., Cheek M., Degreef J., Faden R.B., Goldblatt P., Maesen L.J.G. van der, Ngok Banak L., Niangadouma R., Nzabi T., Nziengui B., Rogers Z.S., Stévart T., Valkenburg J.L.C.H. van, Walters G. \& de Wilde J.J.F.E. 2006. Checklist of Gabonese Vascular Plants. National Garden of Belgium.

The Plant List. 2013. Ver. 1.1. Available from http://www.theplantlist.org/ [accessed 6 Jun. 2018].

Thiers B. (continuously updated). Index Herbariorum: A global directory of public herbaria and associated staff. New York Botanical Garden's Virtual Herbarium.

Available from http://sweetgum.nybg.org/science/ih/ [accessed 6 Jun. 2018].

Verdcourt B. 2001. Desplatsia. In: Whitehouse C., Cheek M., Andrews S. \& Verdcourt B. (eds) Flora of Tropical East Africa, Tiliaceae: 63-67. Royal Botanic Gardens, Kew, Richmond, Surrey.

Wellsow J. \& Harris D.J. 2019. A taxonomic revision of the African genus Desplatsia Bocq. (MalvaceaeGrewioideae). Dataset: v20191127; publisher: Staatliche Naturwissenschaftliche Sammlungen Bayerns - SNSB IT Center, München. https://doi.org/10.25897/5/2war-9p81

White F. 1979. The Guineo-Congolian region and its relationships to other phytochoria. Bulletin du Jardin botanique national de Belgique 49: 11-55. https://doi.org/10.2307/3667815 
Wilczek R. 1963. Tiliaceae. In: Robyns W. (ed.) Flore du Congo, du Rwanda et du Burundi. Vol. 10: 40-46. L'Institut national pour l'études agronomique du Congo (I.N.E.A.C.), Bruxelles.

Manuscript received: 16 February 2019

Manuscript accepted: 12 September 2019

Published on: 5 December 2019

Topic editor: Frederik Leliaert

Desk editor: Radka Rosenbaumová

Printed versions of all papers are also deposited in the libraries of the institutes that are members of the EJT consortium: Muséum national d'histoire naturelle, Paris, France; Meise Botanic Garden, Belgium; Royal Museum for Central Africa, Tervuren, Belgium; Royal Belgian Institute of Natural Sciences, Brussels, Belgium; Natural History Museum of Denmark, Copenhagen, Denmark; Naturalis Biodiversity Center, Leiden, the Netherlands; Museo Nacional de Ciencias Naturales-CSIC, Madrid, Spain; Real Jardín Botánico de Madrid CSIC, Spain; Zoological Research Museum Alexander Koenig, Bonn, Germany; National Museum, Prague, Czech Republic. 